\title{
Modelling the Innovation Process: A Multi-Case Comparison
}

\author{
Fern Evitt
}

A thesis submitted in partial fulfilment of the requirements for the degree of Master of Business Innovation and Entrepreneurship, Unitec New Zealand, 2007 


\begin{abstract}
This thesis studies the management of the internal innovation process. Innovation is considered critical to success in business today, yet companies do not always unlock the value contained within innovations. The purpose of this study is to develop an understanding of the practical systems currently employed by companies and to gain insight into the activities leading to successful innovation outcomes. The expectation is that a more structured approach to innovation management would deliver greater effectiveness to realising innovation value.

A multiple case study strategy was utilised comparing and analysing three companies' existing innovation-to-outcome systems, with Rogers' Innovation Decision Process model adopted to ground the study. The findings advance knowledge of innovation system events and related features with results revealing two main innovation systems as consistent across the companies studied. There is a basic system for realising incremental innovation as a consequence of an organisation's ethos for continuous improvement. The second is a more complex system for radical innovations. This second system supplements the basic version by providing safeguards against the risk inherent nature of this type of innovation.
\end{abstract}

The research indicates that a successful innovation-to-outcome system does not operate in isolation. Rather it appears that there is a link between companies' operating environments and the effective realisation of innovation value. Further, the results suggest that for such companies successful unlocking of innovation value can lead to growth. This in turn requires formalising innovation systems to sustain innovation activities. This study offers an emerging input/output model drawing on insights and referencing patterns associated with best practice. The model provides a basis for companies to formalise the management of their innovation activities. 


\section{DECLARATION OF WORK}

Name of Candidate: $\quad$ Fern M Evitt

This thesis is submitted in partial fulfilment for the requirements for the Unitec degree of Master of Business Innovation and Entrepreneurship at Unitec New Zealand, 2007.

\section{Candidate's Declaration}

I confirm that:

- $\quad$ this thesis represents my own work,

- the contribution of any supervisors and others to this work was consistent with the Unitec Regulations and Policies,

- $\quad$ research for this work has been conducted in accordance with the Unitec Research Ethics Committee Policy and Procedures, and has fulfilled any requirements set for this project by the Unitec Research Committee Ethics Committee Approval, Number: 2006.542.

Candidate signature Date:

Student number: 1233739 


\section{ACKNOWLEDGEMENTS}

Firstly, I would like to convey my appreciation to the companies who kindly agreed to be case studies. Without the generous contribution of both the companies and individual participants, this study would not have been possible.

A sincere thank you to my supervisors Dr Simon Peel and Dr Donna Henson for their advice, guidance and high standards, all of which I found invaluable. To the various staff members at Unitec who each contributed nuggets of wisdom, my thanks for helping build my knowledge. Also, my thanks to A Houghton for a well-presented MBIE research project which I found most helpful as a guide.

I would also like to acknowledge the wonderful support of Dr Coral Ingley whose generous assistance and encouragement kept me on track. Also, my grateful thanks to my family and friends for their interest, support and caring.

Finally, and most importantly, I would like to convey my deepest gratitude and admiration to my husband Michael Griffith. His words of encouragement and unfailing support, his patience and understanding helped to carry me through and often inspired me.

I dedicate this thesis to my father, Colvin Preston Evitt, who passed away during the course of this study. He demonstrated to me the value of tenacity and being true to oneself. He also taught me the importance of being innovative long before the concept was popular. 


\section{TABLE OF CONTENTS}

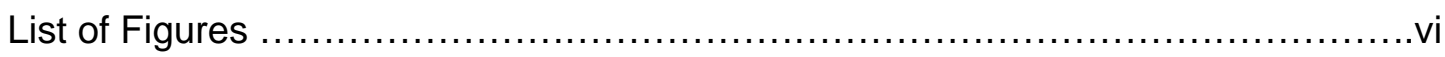

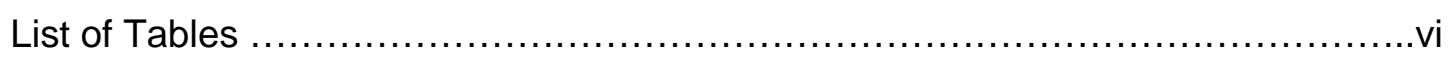

Chapter

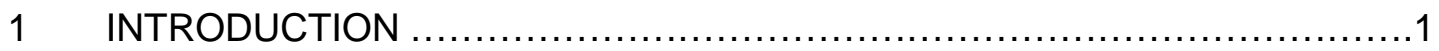

Innovation and its importance in business today ........................ 1

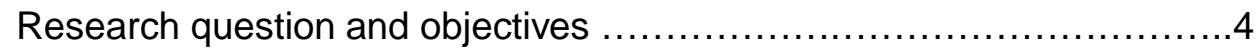

2 LITERATURE REVIEW

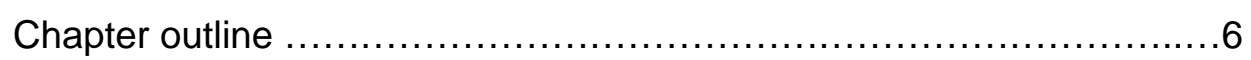

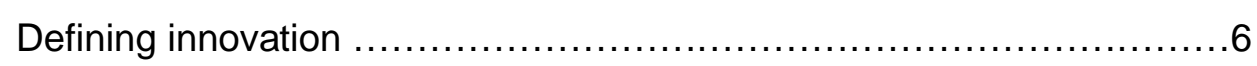

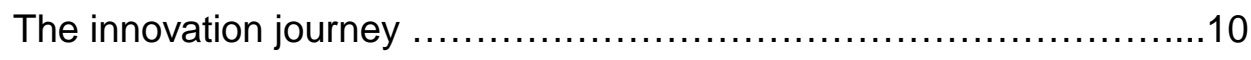

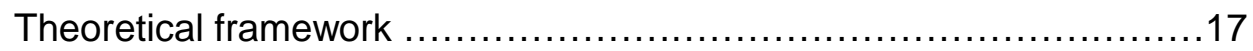

Managing innovation features and practices ..........................22

Provisional steps in the innovation journey .............................24

Influences on the innovation journey: barriers and supports ............25

3 METHODOLOGY

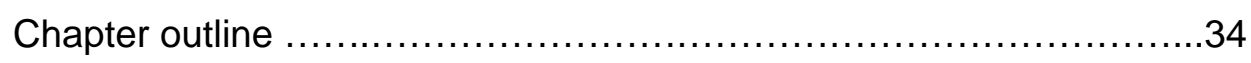

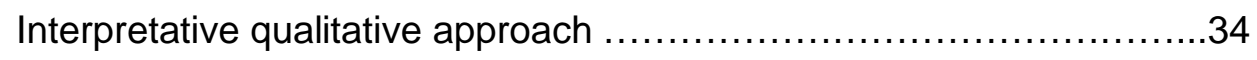

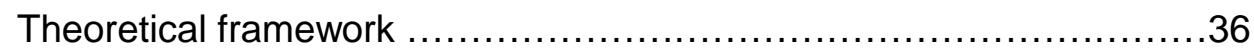

Election of case study as research strategy ..........................36

Concerns with method selection ..................................... 39

Selection of industry and case study companies .......................42

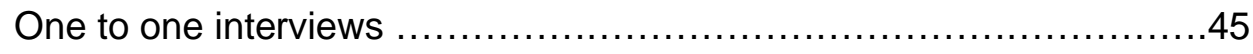

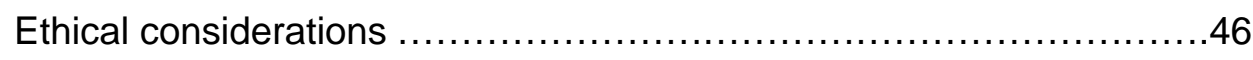

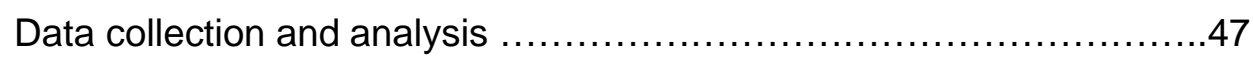

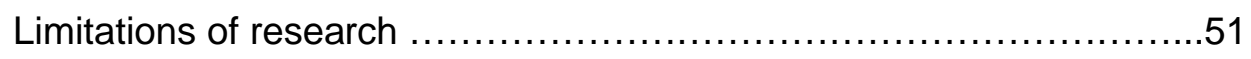

4 RESULTS

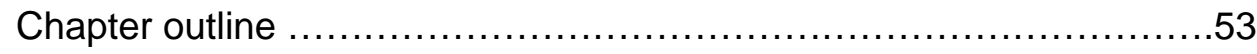

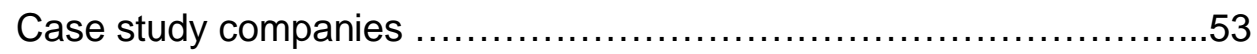

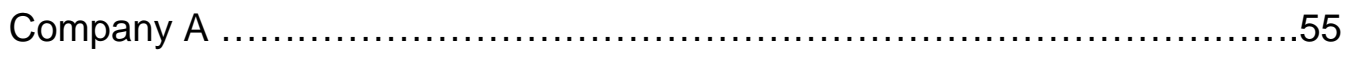

The practical innovation-to-outcome systems employed ................55

Features of Company A's systems .................................61

Company A's practices supporting innovation process management ...62 
Innovation journey influences......................................67

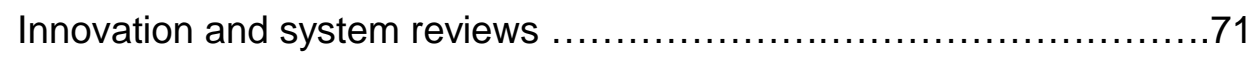

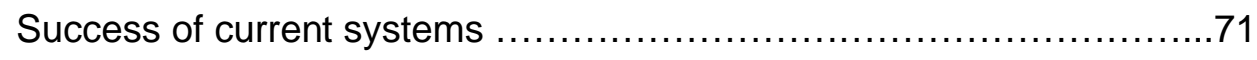

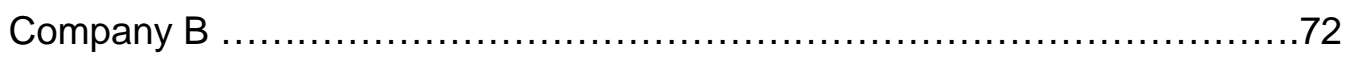

The practical innovation-to-outcome systems employed ...............72

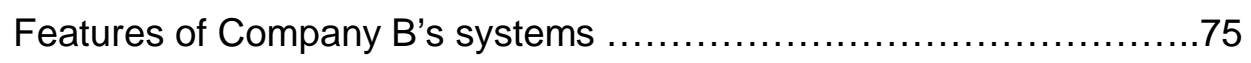

Company B's practices supporting innovation process management....77

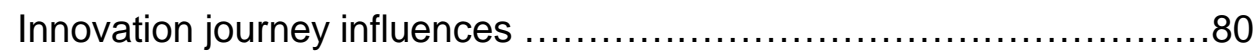

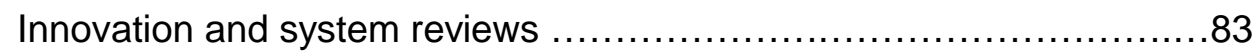

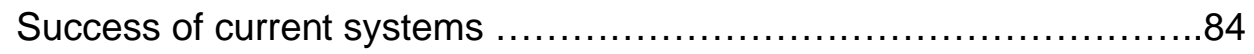

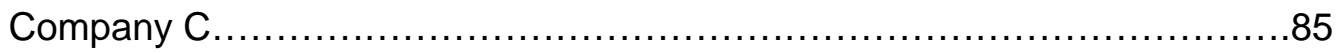

The practical innovation-to-outcome systems employed ...............85

Features of Company C's systems ................................. 89

Company C's practices supporting innovation process management ...90

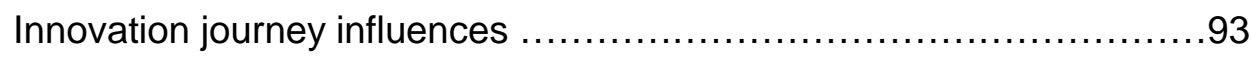

Reviewing and formalising of Company C's innovation systems.........97

Success of current systems .....................................97

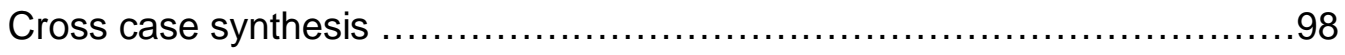

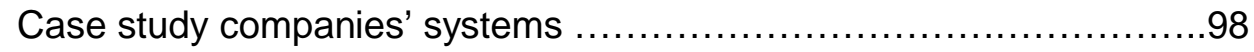

Practices supporting innovation realisation ..........................100

Innovation journey influences ...................................100

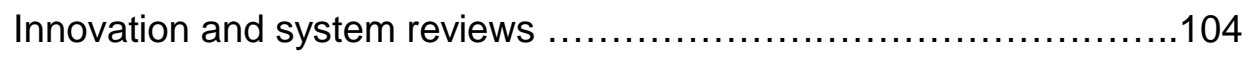

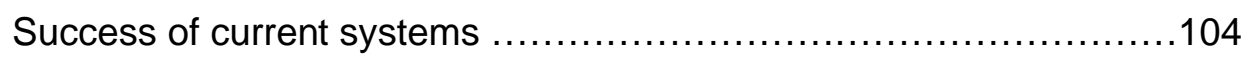

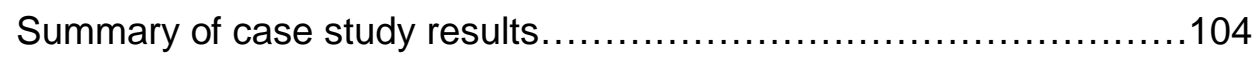

\section{DISCUSSION}

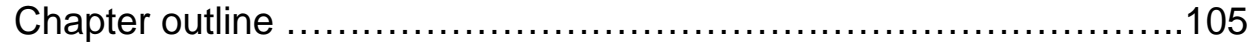

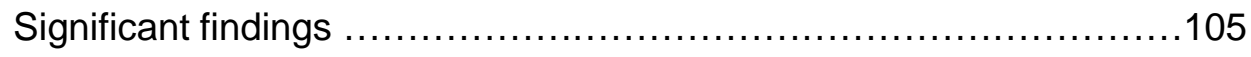

Innovation journey influences ....................................111

Innovation to outcome system events .............................112

Emerging models ..................................................117

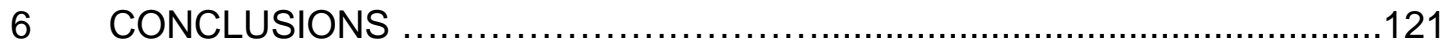

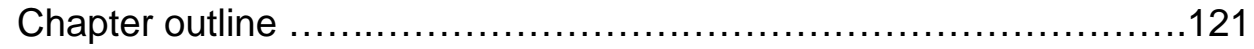

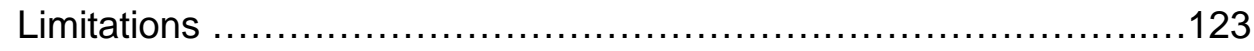

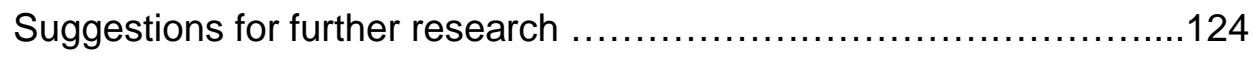

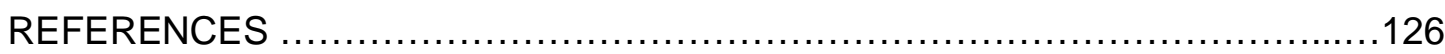




\section{LIST OF FIGURES}

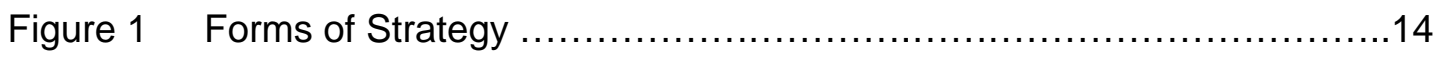

Figure 2 Adaptation of Forms of Strategy ..................................15

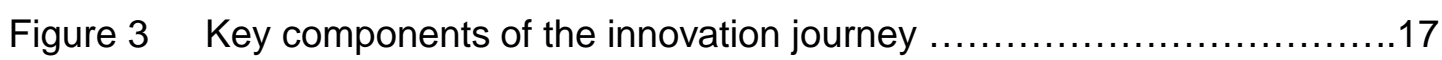

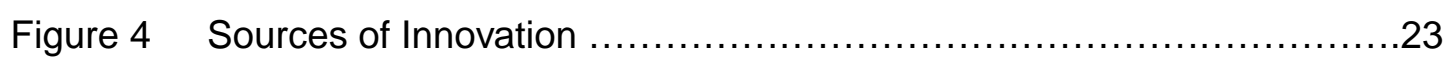

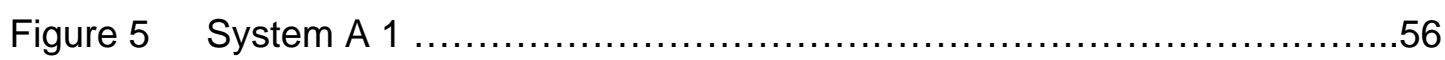

Figure 6 System A 2: Ideas sourced via company intranet .....................57

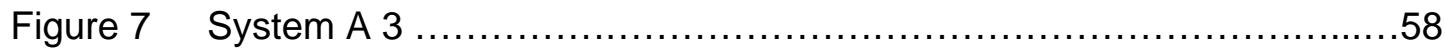

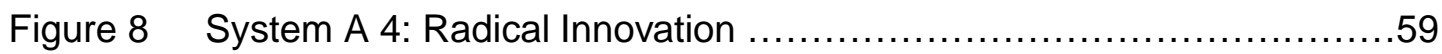

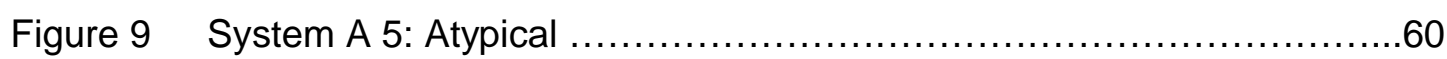

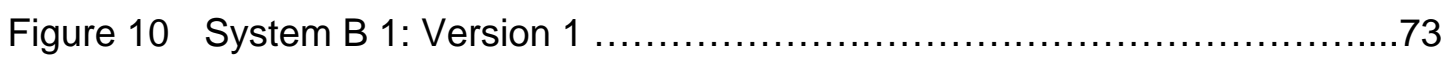

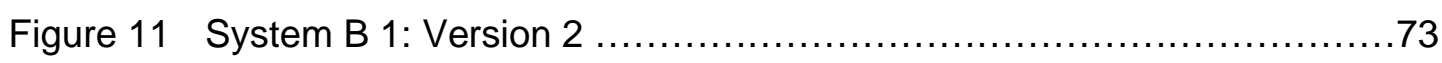

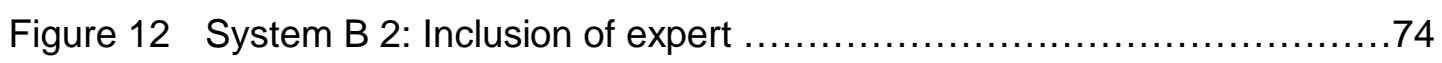

Figure 13 System B 3: Example of system for radical innovation ..................75

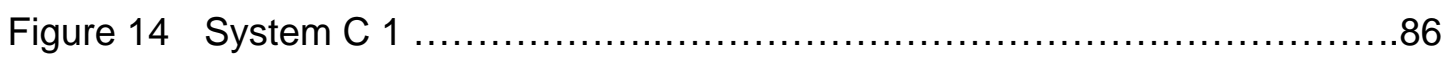

Figure 15 System C 2: Radical or resource demanding innovations ...............87

Figure 16 System C 3: New innovation system (in development) ..................88

Figure 17 Model 1: Innovation sphere of influence ............................118

Figure 18 Model 2: Emerging innovation journey system........................119

Figure 19 Model 3: Emerging system with innovation spheres of influence........120

\section{LIST OF TABLES}

Table 1 Potential stages in the innovation-to-outcome journey ...................25

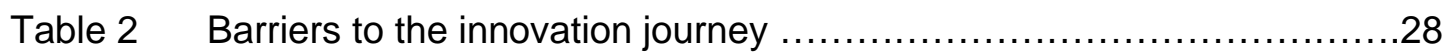

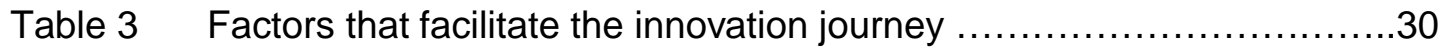

Table 4 Company A Interviewees' responses to barriers .......................68

Table 5 Company A Interviewees' responses to facilitators .....................70

Table 6 Company B Interviewees' responses to barriers ........................81

Table 7 Company B Interviewees' responses to facilitators ......................83

Table 8 Company $C$ Interviewees' responses to barriers .........................94

Table 9 Company $C$ Interviewees' responses to facilitators .......................96

Table 10 Compilation of case study companies' systems ........................99

Table 11 Combined companies' barriers to innovation outcome ...................101

Table 12 Combined companies' facilitators to innovation outcome ................103 


\section{LIST OF APPENDICES}

Appendix A Information Sheet for interview participants

Appendix B Consent Form

Appendix C Interview Guideline

Appendix D Lists of barriers and supports provided to interviewees

Appendix E Compilation of case study companies' basic systems

Appendix F Compilation of case study companies' radical systems

\section{ABBREVIATIONS}

NPD New Product Development

R\&D Research and Development (department)

ICT Information Communication Technology 


\section{Chapter 1 \\ INTRODUCTION}

\section{Innovation and Its Importance in Business Today}

As the business world becomes increasingly competitive and the effects of globalisation and technology intensify, companies are striving for new ways to create competitive advantages. In this context, the drive to innovate is vitally important. Companies must innovate to create or improve new products and services, enhance systems, develop more effective business models, produce superior marketing efforts.

In particular, New Zealand businesses need to be innovative to overcome their lack of proximity to international markets and to compete within the country's small arena. Statistics New Zealand's Innovation Survey (2004) found that businesses which implement innovations reported greater profitability (79 percent), increased markets (64 percent), and improved efficiency (75 percent), providing evidence of innovation's impact on a company's bottom line (Jones, 2002b) and its ability to stimulate growth.

The importance of innovation as a mechanism for growth is well documented. As a driver of sustainable success and even survival, companies need to innovate to compete effectively in business (Cooper, 1998; Cooper, 2005a; Hamel \& Prahalad, 1994; Jauhari, 2001; Kaplan \& Norton, 1992). This is corroborated by Van de Ven and Engleman (2004) (citing Schumpter (1942)) that innovation is important for business and society alike, postulating that there is little argument as to the importance of innovation's role in economic development.

The European Union has recognised the significance of innovation to economic prosperity by introducing policies encouraging and supporting innovation activity (Commission of the European Communities, 2003). Further, results of the IBM Global CEO Study (2006) indicate industry is shifting focus increasingly to innovation to provide differentiation and growth. Companies large and small are actively encouraging innovation through generating and supporting inventions, creative thinking and bright ideas - all initiators or drivers of innovation - to help differentiate and compete (Sharma, 1999; Statistics New Zealand, 2004; Thornberry, 2001). 
However, stimulating innovative ideas is not necessarily the challenge companies face. The Boston Consulting Group's (2005) worldwide survey identified many companies as having "more ideas than they can effectively pursue" (p. 9). The survey findings go on to state that over half the respondents reported their company as being weak or very weak in bringing innovations to fruition, or realising the value contained within innovation.

Drucker (1985b) contends that innovation is the creating of a resource, a resource that does not exist until furnished with economic value achieved through capturing the potential of an innovation. This means that regardless of the promise an innovation may appear to provide to a company, without realising that promise by transforming the innovation into a usable outcome - harvesting its potential - the innovation remains nothing more than a good idea. Despite the importance of this, the transforming of concept to outcome is not readily achieved, hence, the value inherent in many innovative concepts is not always realised by companies.

An extensive body of literature exists on the conceptual processes or systems an organisation experiences in attempting to realise innovations (for example see Schroeder, Van De Ven, Scudder and Polley's comparative study (1986)). The innovation process is defined by Van de Ven, Polley, Garud and Venkataraman (1999) as occurring when inventing, developing and implementing new programmes, services, and products. However, there is little empirical evidence on what constitutes the various stages in the journey in terms of practical considerations. There is an absence of prescriptive indications or step-by-step instructions that a company may adopt. There are general discussions and theoretical guides provided in literature, however, these do not provide actual series of steps that a company may adopt. Anecdotal evidence suggests that this conversion of innovation into a useful outcome to a company is, at best, an idiosyncratic process, unique to each firm. That innovation at all is realised through the "labyrinthine world of conflicting forces within an organisation" (Bannon \& Grudin, 1990 p.49) is remarkable.

The systematic activities undertaken by a firm in transforming innovations into useful outcomes remains vague. Understanding what influences the process is also unclear, specifically, what internal barriers prevent realising innovation value. The Council on Competitiveness' National Innovation Survey (2005) refers to the key challenge in realising innovation as these internal barriers. This inability to actualise 
the potential of an innovation owing to the internal process and barriers met therein, Morrissey (2000) proposes, is a point of consternation to businesses.

A plethora of articles exist on implementing innovation in a firm, for example, Bean and Radford (2002), Christensen and Raynor (2003), McAdam (2005), Philips and Hering (2005), Toftoy and Chatterjee (2004). However, on analysis these articles refer to creating and sustaining the innovative company not functional or practical steps a firm can employ for realising innovation. Similarly, studies on generic process models (Schroeder et al., 1986) investigate activities without revealing what comprises workable steps in innovation systems. Van de Ven and Angle (1986) agree that the information obtainable on innovation systems "lacks empirical validity" (p. 5). What is available is information to assist the passage of the planned innovation. Most often these are new products, innovations eventuating from research and development or outputs of intentional actions by a company perceiving an opportunity.

Planned innovations are expected innovations, where a company identifies and takes steps to address a gap in their product or service range, usually with an external aim, with resources allocated and processes established to maximise return on investment. What is not clear is what happens with the unplanned innovative concept, the innovations that originate from unexpected sources, or at unexpected times that have unanticipated demands on a company. Similarly, what happens with the radical innovation, innovations that have dramatic implications to a firm. What are the practical steps on this path of transforming the concept into an outcome of economic value to the business? An employee has an innovative idea, which may be self-generated or inspired externally that promises to be of value, what then occurs? For instance, is the concept taken to the line manager? Or is there an established innovation team that works on feasibility, with or without the idea generator? Is a business or viability plan written, if yes, what is included?

Despite challenges, companies are nevertheless achieving innovation outcomes, albeit perhaps not as often as desirable. This is reinforced by the BCG (2005) and the Council on Competitiveness' (2005) surveys that suggest potentially valuable innovations are not being realised. The challenge is not singular as competing through innovation is not an isolated event. In developing new processes, products and systems, a company stimulates others to react. Often competitors respond to innovation developments in an industry by engaging in 
innovative activities themselves. Hence to maintain consistent and effective competitive advantages a firm must adopt innovation imperatives (Cooper, 1998), regardless of the difficulty or lack of understanding of how to best realise innovation value.

\section{Research Question and Objectives}

This seeming gap in empirical evidence on the process of transforming innovation concepts into an outcome (thereby harvesting an innovation's value), is the focus of this research. The research question therefore is "How can the process of managing innovation from inception to outcome be modelled?" with the research objectives as follows:

1. To model the path of innovation through the event sequence from inception to completion or abandonment.

2. To identify from examination of these innovation models any patterns or common practices that typify the management of transforming innovation.

3. To determine any patterns and practices hindering the process reaching a successful outcome.

4. To determine any patterns and practices associated with successful, value-creating outcomes.

5. To develop a framework that will provide useful as a basis for guiding best practice in innovation management

In order to answer the question and meet objectives, this study investigates how an innovation proceeds from inception to implementation internally through a company, examining actual innovation systems developed by organisations. This study expects to lead to better understanding of the steps in the journey innovations undergo. As well, to generate insights into the factors and influences associated with successful outcomes.

It is possible that greater success could be realised in transforming innovations from conception into being if there was improved understanding of the processes developed for this purpose. Rather than an organisation simply employing an ad hoc approach, it could be that greater understanding of currently implemented systems would help a business identify key factors for success, leading to systematic planning and execution for greater effectiveness. Patterns leading to successful 
innovations and the modelling of best practice for managing the innovation process may also be revealed through improved understanding innovations.

This chapter has introduced the overall situation regarding innovation in business and the importance of realising the value contained in innovative concepts. Realisation of value relies on effective internal innovation-to-outcome systems which currently appear to be ambiguous. Following, Chapter Two reviews current literature on this subject, investigating various models and existent opinions providing the context for this study.

Chapter Three details the research method chosen to address the study's question. Employing a multiple case study strategy, Chapter Four reports the results of interviews and other data gathering on three companies' innovation-to-outcome systems. These systems are compared and analysed to develop understanding of contemporary processes. Chapter Five then presents key findings considering various interpretations and theoretical precepts introduced in Chapter Two's literature review. Academic theories, writings and models are employed in order to critically evaluate findings and provide better comprehension of case study companies' existing practices. Finally, Chapter Six provides conclusions and considers the theoretical and practical applications of this study, identifies study limitations and recommends further areas for research. 


\section{Chapter 2 \\ LITERATURE REVIEW}

\section{Chapter Outline}

The chapter firstly considers the many meanings of innovation and related concepts, giving necessary definitions to frame this study. Next, although direct evidence of empirical studies on functional events in the innovation-to-outcome journey was not discovered during this literature review, related areas are examined, presenting alternative views that help to clarify possible practices. This study then draws on strategic planning as an analogy, arguing that as this complex discipline, unique to a business can be formalised supports the realisation of a practical model of the innovation journey.

The interrelated areas of continuous improvement and change management are discussed as related to implementing innovation in an organisation. The theoretical construct employed for this research is then introduced together with consideration of existing process models including new product development. Next, various steps suggested by literature are presented as providing possible events in innovation-to-outcome systems. Finally, examples of obstacles to and enablers of innovation in firms are drawn from complementary readings. These are translated to the internal process as indicating potential influences on the innovation journey.

\section{Defining Innovation}

What is Meant by Innovation

Innovation definitions abound as do interpretations of forms of innovation (ranging from new products through to business model innovations), types of innovation (incremental through to radical), and results of innovation (from launching a new product to overhauling a company structure). The various meanings of innovation and related concepts are widely debated. Various empirical definitions are offered below, providing parameters to assist comprehension of the variances of innovation and its outputs, and how these concepts are defined for this study.

Practitioners and researchers define innovation in different ways (Cooper, 1998; McFadzean, O'Loughlin, \& Shaw, 2005). Jauhari (2001) stipulates that definitions for terms such as innovation have not been widely accepted, a point of view reinforced by Statistics New Zealand (2004). However, the Commission of the 
European Communities (2003) offers the following, defining innovation as: "The successful production, assimilation and exploitation of novelty in the economic and social spheres." (p. 5). McFadzean et al (2005) contend that there is some confusion as to what constitutes innovative activity providing their interpretation:

Innovation can be defined as a process that provides added value and a degree of novelty to the organisation and its suppliers and customers through the development of new procedures, solutions, products and services as well as new methods of commercialisation. (McFadzean, O'Loughlin \& Shaw, 2005, p.9).

For the context of this study, innovation is synonymous with invention (Cooper, 1998; Weller, Green, \& Fernie, 2004), and creativity (Drucker, 1985b; McAdam, Stevenson, \& Armstrong, 2000; Peters \& Waterman, 1982; Williams, 1999). Allen (2003) argues that innovation differs from invention as innovation is the process that transforms the invention into something of use. In other words, innovation is the application of the invention. However, she goes on to say innovation is "about creating new ways of doing things" (p. 8) referring to innovations' originality, hence providing little distinction between the two concepts. Zaltman's (1973) definition encompasses several facets defining innovation as firstly, the invention or creation of a concept, secondly the implementation or adoption process.

Weitzel and Hallahan (2003) state that innovation can be a (perceived) original idea; an idea new to the adopting organisation or the combining of existing concepts in a novel way (Statistics New Zealand, 2004). Van de Ven (1986) and Cooper (1998) clarify this further by proposing that an innovation may be something new to a company, even if in application elsewhere, therefore newness of a innovative concept being a matter of perception. Rogers (2003) concurs, his definition of innovation is "an idea, practice, or object perceived as new by an individual or other unit of adoption" (p.35).

McAdam, Stevenson and Armstrong (2000) add another dimension to defining innovation, drawing on various definitions including Drucker (1985a) and Peters and Waterman (1982), by stipulating that the meaning of innovation includes change and creativity. Whereas Tidd, Bessant and Pavitt (1998) simply state that innovation is essentially change. 
The definition of innovation used in this study also corresponds with the term corporate innovation, which is the generating of innovations within an existing business. The definition also includes Intrapreneurship (Pinchot, 1985) and Corporate Entrepreneurship referring to entrepreneurial-style activities inside a company (Batiz-Lazo \& Woldesenbet, 2006a; Bouchard, 2001; Frederick, 2006). These two activities often occur as catalysts for innovation (McFadzean et al., 2005). Batiz-Lazo and Woldesenbet (2006a) and Bouchard (2001) define intrapreneurship and corporate entrepreneurship as where an existing organisation, in conjunction with an individual or group of individuals, creates a new business or initiates innovation within that organisation. The broad range of definitions provides some parameters as to what can be construed as innovation.

\section{Forms of Innovation}

Innovations come in many forms, whether tangible as in new products, or intangible: for example as new services and work practices. Forms of innovation are described by Bean and Radford (2002), who list innovations as products (new or improved), processes, management and organisational developments. These are also identified by Birkenshaw, Hamal and Mol (2005) adding strategic innovation to the list of innovation forms and define management innovation as being intended to further organisational goals through "creation of a new management practice, process or structure" (p.7). Davenport, Prusak and Wilson (2003) propose that managerial innovations are more sustainable - and stronger differentiators - than product innovations as managerial innovations are crucial support activities to product innovation outcomes (Birkinshaw et al., 2005).

Further forms are service and business model innovations, referred to by Hamel and Getz (2004) together with Davenport (2006), whilst Papadakis and Bourantas (1998) amongst others (for example Hesselbein, Goldsmith and Sommerville (2002)) identify technology and organisational innovations. The list continues including administrative innovations (Cooper, 1998) and management principles (Hamel, 2006). Finally, the Commission of the European Communities (2003) refers to business model innovation, technology innovation, organisational innovation (new methods for organising work), and presentational innovation (design and marketing innovations). 


\section{Types of Innovation}

Allen (2003) cites Christensen (1997) as recognised for identifying different types of innovation. Firstly radical innovations, innovations that dramatically change a firm's activities making obsolete previous ways of operating and changing an organisation's order (Van de Ven et al., 1999). These are referred to by Birkenshaw et al (2005) as disruptive innovations which challenge the current status of a firm, whereas McFadzean et al (2005) call them frontier innovations. Pavitt (1991) refers to these types of innovations as revolutionary, breakthrough or discontinuous. This type of innovation is considered high risk (Thornberry, 2006; Tidd et al., 1998; von Stamm, 2003) and exists at the extreme of the innovation continuum. In adopting these types of innovation a company's traditional practices will most likely undergo extreme change. For instance, the internet is considered a transformative innovation. Dramatic changes to current business models are required for organisations desiring to operate in this arena (Cooper, 1998; von Stamm, 2003).

At the other end of the continuum there exists a more modest type of innovation. The sustaining innovation meets a company's ongoing demand for constant improvement (Allen, 2003; Williams, 1999). Also known as incremental innovations (Birkinshaw et al., 2005; Pirich, Knuckey, \& Campbell, 2001; von Stamm, 2003), enhancing or derivative innovations (Thornberry, 2006) these types of innovations build on and refine existing practices. Between these two extremes of innovation types lie other types referred to by Thornberry (2006) as platform innovations. These are slightly more risky than incremental innovations but do not have as much impact as radical.

\section{Results of Implemented Innovations}

Finally, the result of successfully unlocking an innovation's value is referred to in this study as outcome, actualisation, or realisation of an innovation's asset. The nature of innovation outputs can take many forms not necessarily resulting in a new product entering the market, which is known as commercialisation (Cooper, 2001; Statistics New Zealand, 2004; Tarrant, 2005b). Commercialisation is the focus of numerous writings, for example Cooper (2001; 2005b) and Annacchino (2003) have centred on assisting businesses transform their product innovations to marketreadiness.

Other outputs are the creation of a new entity separate to an existing organisation or corporate venturing (Frederick, 2006). IBM refers to these as EBOs, 
Emerging Business Opportunities (Hamel, 2000). An outcome may also result in implementing change to a company's operating or management structure (for example in adopting an innovative business framework), entry into new markets or providing new services (Govindarajan \& Trimble, 2005; Sathe, 2003; Thornberry, 2001), or simply as McAdam et al (2000) sum it "doing things differently or better" ( $p$. 140).

This multitude of innovation meanings and outcomes of innovation increases ambiguity and complexity in understanding and defining innovation. However, regardless of how innovation is described, innovation impacts on numerous facets of a business. Innovations, through creativity and originality of ideas, add new dimensions to companies which are then realised within organisational confines.

\section{The Innovation Journey}

An innovation moves through an organisation undergoing a journey to reach an outcome. Understanding the innovation journey is important to assist businesses realise greater effectiveness in unlocking the value contained in innovation. That innovation is significant to business today is supported by numerous sources.

Academic literature and business publications emphasise the need for organisations to innovate with bold statements: "We hold this truth to be self-evident: that innovation is the life force of a living, adapting organisation" (Bean \& Radford, 2002. p. 289) and "It's war: Innovate or die" (Cooper, 2005a, p. 4). Other articles focus on facets of corporate innovation such as the need to stimulate employees' creativity (Harvard Business Review, 2002; von Stamm, 2003) and driving innovation strategy (Tarrant, 2005a) for example.

Research of literature on the practical internal innovation process uncovered little evidence of directly relevant information on functional systems. Therefore, comparable and pertinent topics are considered for edification including literature from the fields of communication, information systems and creativity. Weller, Green and Fernie (2004) propose that when considering innovation within a business environment it is often integrated with other issues such as the above, together with change management and organisational development. Strategic planning was identified as a similarly difficult trial-and-error procedure, thus having likely parallels and potentially offering insights into the internal innovation process. Stages proposed to expedite new product development were also investigated as a means of understanding potential steps. 
New business start-ups were examined as possibly sharing similarities in processes. However, the differences between an entrepreneur establishing a new venture and the innovation journey were seemingly fundamental, restricting relevance. For instance, the often bureaucratic and risk-adverse nature of an existing organisation is not usually present in a new business (Sharma, 1999; Thornberry, 2001). Similarly, small entrepreneurial companies have flexibility not generally present in larger companies. Conversely, they do not have access to the resources available in larger and more established firms (Sharma, 1999).

\section{Innovation and Business}

The subjects of corporate innovation and achieving an innovative organisation have been increasingly prevalent over the last five years. However, older roots exist: seminal works such as Drucker's "Innovation and Entrepreneurship" (1985b), "The Fifth Discipline" (Senge, 1992) and the "Balanced Scorecard" (Kaplan \& Norton, 1992) led thinking on the significance of organisations' practices regarding innovation. Van de Ven's extensive study of innovation is complemented by his and his colleagues' work on the Minnesota Project (1999) summarising 17 years of research into the innovation journey and the pertinence of innovation to today's business world.

The criticalness of innovation in companies and its importance in sustaining growth is referenced in the works of Hamel and Getz (2004), Handy (2002), Kaplan and Norton (2000) who provide guidance on how to realise the innovation-orientated company or the developing of innovation capability (Jones, 2002b). Similarly, how to grow an organisation innovatively by Christensen (1997) and Christensen and Raynor (2003) provide guidelines aiming to support companies desiring this ability. Srivastava and Gnyawali (2006) reinforce the importance of innovation today to "thrive and even survive" (p.3) discussing innovation as a primary source of sustainable competitive advantage. Additionally, the importance of an innovation strategy is demonstrated by Hamel and Prahalad (1994), Kaplan and Norton (2000), and Kanter (1989) who stipulate the relevance of innovation to a firm's sustainability and strategic intent. Finally, the need to differentiate an organisation's offering achieved through innovation activity is discussed in particular by Jones (2002b), Sharma (1999) and Thornberry (2001) underpinning the necessity of companies to unlock the value contained in innovation. 
The part played by innovation in a business may be subtle. Continuous improvement practices are often the result of incremental innovations (Birkinshaw et al., 2005; Pirich et al., 2001; Williams, 1999), or as perceived by Weller, Green and Fernie (2004) the two are "invariably intertwined" (p. 1). Weller et al, observe that the word innovation is often interchanged with knowledge creation, organisational change and continuous improvement. Their conclusion is that incremental innovation, as the catch-all concept for regular occurring small scale, almost inconspicuous changes, would be difficult to separate from continuous improvement. Nonaka and Takeuchi (1995) and McAdam, Stevenson and Armstrong (2000) agree, directly linking knowledge creation with continuous innovation to realise or sustain competitive advantage. As well, McAdam, Stevenson and Armstrong (2000) argue that companies that have a continuous improvement philosophy create innovative organisations.

\section{The Problem}

Pavitt (2003) argues that innovation and its processes are contingent, differing from company to company owing to the vagaries of their activities and business/industry environment. He contends that these contingencies together with the heterogeneous nature of innovation hinder the creation of a simple best practice model for companies. There is some empirical evidence supporting this view. Schroder et al's (1986) Minnesota Innovation Research Program compared numerous existing conceptual innovation process models, from simple to complex, to establish a better theoretical framework. They propose that most models are based on decision-making systems for groups or individuals, organisational planning, or change and development rather than innovation system requirements. Further, they consider these models as often having a unitary process and lack sophistication to address the complexity of innovation.

Further, Pavitt (2003) dislikes the use of words such as process, steps or stages as they impose a linear aspect on the procedure, reinforcing his stance that the innovation-to-outcome process is vague and chaotic. He believes that progress through an organisation is more likely to overlap and the events mingle. He goes on to argue that innovation sub-processes often happen concurrently. Previous publications support Pavitt's (2003) view (Schroeder et al., 1986; Van de Ven et al., 1999; Van de Ven \& Rogers, 1988) that innovation is the antithesis of linear activity. 
Schroder et al (1986) in rejecting the sequential, consecutive progress presented by conceptual innovation process models, explain that they lack empirical correctness or validity in proposing such regimented sequences. This view is also supported by Birkenshaw, Hamal and Mol (2005) who propose that processes be non-sequential with overlapping phases, an opinion also shared by Nutley, Davies and Walter (2002), Pirich, Knuckey and Campbell (2001) and Van de Ven (1993). Cheng and Van de Ven (1996) also corroborate with these views. However, they propose that portions of the journey may be methodical, whilst others chaotic, providing a dynamic platform for further development by a firm. Cooper (1998) stipulates that innovation could be regarded as either a process or a discrete event, whilst arguing that differences are most evident when the innovation is put to use.

However, the models analysed by this study do not appear to investigate practical actions a company must pursue in the transforming of innovation into "good currency" (Van de Ven \& Engleman, 2004). The numerous models available are redundant when considering pragmatic prescriptive instructions a company needs to consider. The more complex non-linear models, in attempting to realistically illustrate the process, also provide little such guidance. As Schroder et al (1986) demonstrate by their comparison of innovation processes in the Minnesota study, the various offerings are much alike providing encompassing guidelines, indicative steps but not functional devices. Lack of specification of what actually does constitute an innovation model contributes to the confusion and is reinforced by poor definition of what innovation means (Cooper, 1998).

\section{Modelling Strategy to Support Premise}

Strategy is a difficult process with unique attributes and characteristics individual to a company, yet it has been successfully mapped. This paper draws on strategy as an analogy to the innovation journey contending that mapping of the innovation process may be similarly achieved. In particular, Kaplan and Norton (1992) and Mintzberg (1994) have modelled strategy from inception through implementation to completion. The strategy process shares similarities with innovation realisation, as do the functional steps in achieving results. Findings of the Minnesota Project (Schroeder et al., 1986; Van de Ven et al., 1999) workshops reinforce this. Managers participating in the workshops shared their experiences of the innovation journey revealing insights and common themes between company strategy and innovation. 
Similarly, von Stamm (2003) stipulates that strategy is directed towards goals that rely on in-depth knowledge of a company and "effective harnessing of the capabilities" (p. 81), both similar to innovation activities. Further, von Stamm (2003) argues that strategy is a plan for action and that innovation often is that action. Hoffman and Hegarty (1993) claim that strategic change occurs when innovation is a source of competitive advantage, again indicating a correlation between strategy and the innovation journey. As well, innovation is often intangible in form as is strategy. However, both have tangible effects on a business further emphasising the similarities between the two.

Given that both strategic planning and the innovation journey involve the conversion of intangible assets to tangible outcomes, a perplexing process facilitated by strategic mapping (Kaplan \& Norton, 2000), it seems reasonable to conclude that a process similar to strategic mapping may be possible for the innovation-to-outcome journey. An example of the similarities between strategic planning and the innovation journey is Mintzberg's (1994) Forms of Strategy (Figure 1). Mintzberg (1994) proposes that there is a pattern to strategy, which the Figure 1 outlines, with emerging strategies contributing to realised strategy, more so than deliberate strategic endeavours. As such, innovation could be substituted for strategy (Figure 2 , p. 15) as innovation concepts also come from a variety of sources and that continuous improvement innovations accumulate to make fundamental changes to a company's operations. Finally, innovations are often individualistic to a company and provide value when realised as does strategic planning.

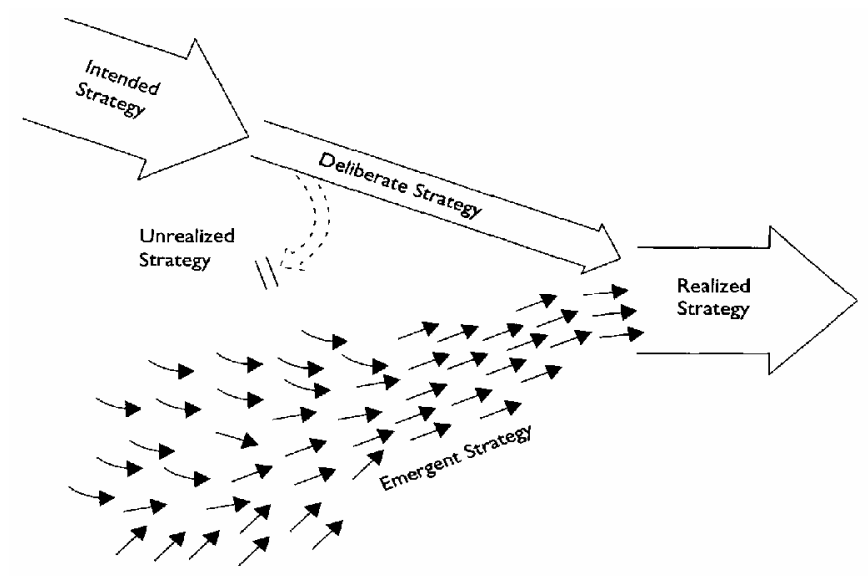

Figure 1: Forms of Strategy (Mintzberg, 1994 p. 24) 


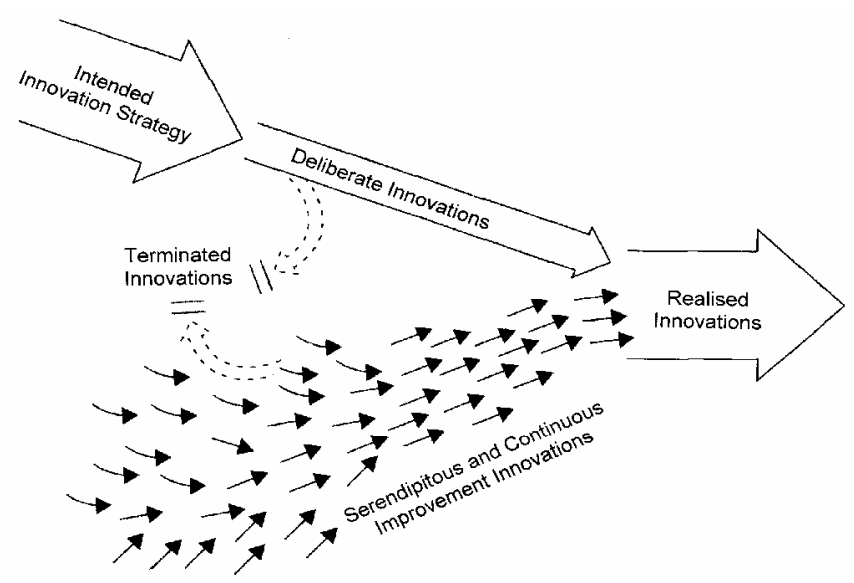

Figure 2: Adaptation of Forms of Strategy (Mintzberg, 1994) showing innovation input sources

\section{Innovation and Organisation Disequilibrium}

Innovation can challenge the very nature of a firm, even more so than deciding strategic direction. Pinchot (1987), renowned for coining the term Intrapreneurship in the 1970s, outlines the interrelatedness between organisational activity and innovation. More specifically his premise is that for a company to successfully innovate it needs to be open to all forms of innovation which he describes as "new ways of working together, new internal services and new organisational patterns" (p.14). Amabile (1998) and Williams (1999) specifically discuss the tension between creativity in an organisation and the meeting of business imperatives including maintenance of clients, daily routine and productivity. Creativity naturally involves introducing a degree of uncertainty. This then countering obsolesce by challenging existing practices (Williams, 1999) and leading to an environment of change.

Hamel and Getz (2004), Kanter (1982) and Pinchot (1987) propose that innovation in an organisation is synonymous with change, generating resistance through challenging a company's status quo. Williams (1999) also agrees with this view stating that change is deliberately introduced as a function of the innovation process. Pinchot (1987) describes this, referring to the corporate immune system as operating when identifying a threat to existing conditions (in other words the change incorporated in innovation) provoking a desire to smother it. This smothering can hinder the actualising of innovations with barriers to innovation realisation purposefully occurring, perhaps as a consequence of a change-resistant culture. Organisational change is often a component of innovation with innovations frequently involving the altering of work practices and processes (Birkinshaw et al., 2005). 
Such changes occur through implementing radical innovation and the collective affect of incremental innovation. Lewin's (1951) precept of the three stages of change (unfreeze, change, refreeze) outlines the change process of unsettling an organisation, introducing change then establishing a new level of stability. The change process thus aligns with the disruptiveness that occurs as a result of implementing an innovation.

The difficulties faced by realising innovation in an established organisation embody Machiavelli's sentiment (1950, p21):

It must be considered that there is nothing more difficult to carry out, nor more doubtful of success, nor more dangerous to handle, than to initiate a new order of things. For the reformer has enemies in all those who profit from the old order of things, and only lukewarm defenders in all those who would profit by the new order, the lukewarmness (sic) arising partly from fear of their adversaries ... And partly from the incredulity of mankind, who do not truly believe in anything new until they have had actual experience of it. Thus, it arises that on every opportunity for attacking the reformer, his opponents do so with the zeal of partisans, the others only defend him half-heartedly.

Hence, an organisation is challenged by innovation in multiple ways owing to its innate creative bias and change basis. This then results in disequilibrium at odds with a company's established order and structure. Resistance to this disequilibrium can lead to hindrances to the innovation process and difficulties in realising outcomes.

\section{Existent Systems and Processes}

Schroeder, Van de Ven and colleagues (1986; 1999) prolific studies on innovation in business and the innovation journey compare a number of theoretical models in order to present an empirically grounded map of how innovations develop. Their findings suggest that the journey is divergent and paths numerous, preventing an orderly sequence. Accordingly they developed a complex, looping process model to map the innovation system events (Figure 3, p.17). Importantly, they state that the innovation journey overall involves the same core processes, regardless of organisation, and that variations of events are not significant. This then supports this study's proposition that it is possible to construct a map of a practical system. 
Further, although the innovation journey is proposed by these researchers as problematic and anomalistic, this does not prevent identifying the tasks, influences and events of systems that would assist companies to better manage innovation outcomes.

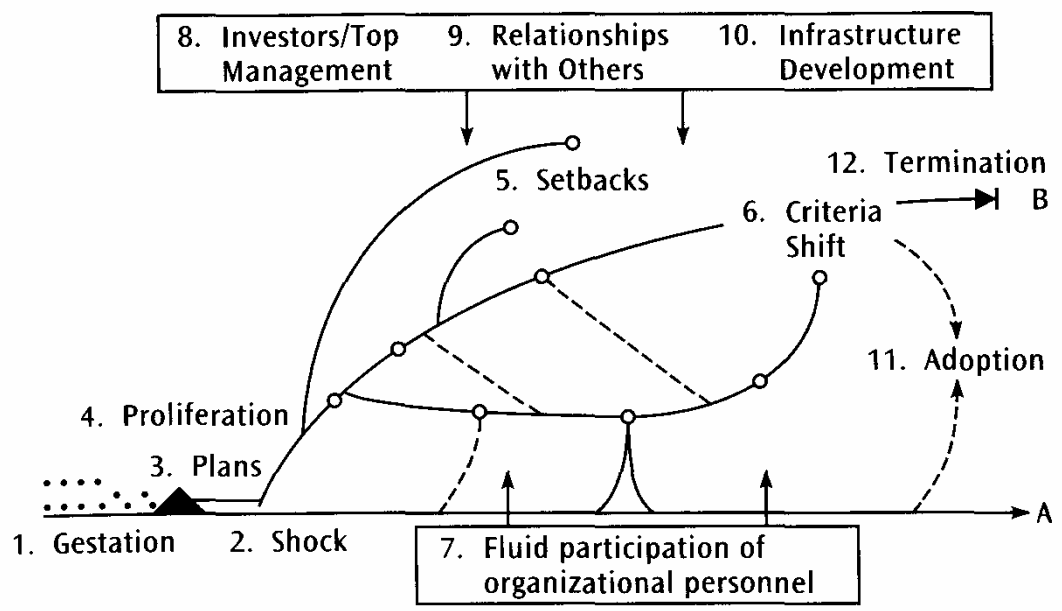

Figure 3: Key components of the innovation journey (Schroeder et al., 1986 p. 25)

\section{Theoretical Framework}

Various theoretical innovation models were examined in order to establish a construct to support this study. These included Cooper's (1998) New Product Development Stage Gate ${ }^{\odot}$ system, Schroeder, Van de Ven, Scudder and Polley's (1986) extensive comparative list and their resulting complex non-sequential model (Figure 3, above); Zaltman, Duncan and Holbeck's (1973) two stage initiation and implementation theory; Majaro's (1998) four step process (as cited in Ahonen 2005); and Williams (1999) more functional offering, which although provides overall steps lacks the practical implications of these (as well, it focuses on purposeful innovations).

Finally considered was von Stamm's (2003) offering which refers to the stages as categories and concludes that variations exist throughout literature of the different development processes, yet the processes remain similar. However, von Stamm's contribution is consistent with the majority of findings on this topic in that his focus is on product innovations. Additionally, conceptual models provided in literature on the innovation journey may describe the implications of each phase and some individual steps but do not offer any series of practicable imperatives. Hence, a company would struggle to recognise a workable system in adopting these theoretical models. 


\section{Employing a Current New Product Development Model}

New Product Development (NPD) models do indicate some workable events including a product plan (Annacchino, 2003; Cooper \& Edgett, 2001) with time periods, feasibility and resource requirements. Cooper's (2005b) perspective is that the success of a new product is the result of a systematic approach based on best practice. His approach is reliant on strategy, commitment of resources to the right projects, the use of the Stage-Gate ${ }^{\circledR}$ system, the right culture and climate, true crossfunctional teams and senior management commitment. Cooper's (2005b) Stage Gate ${ }^{\circledR}$ system for NPD provides a framework consisting of five stages (identified by Cooper and Edgett (2001)): scoping, building the case, development, testing and validation, launch. The framework has five gates as roadblocks where decisions are made as to whether or not an innovation proceeds to the next stage.

However, Cooper's (2005b) and Annacchino's (2003) proposals relate to assisting companies to manage their current NPD activity more effectively. The challenges of realising radical or intangible innovation are not considered. Of significance to this study, also presupposed is an existing R\&D structure and an active product process that needs refining. In the internal innovation process, neither of these may exist in the generic organisation that wishes to realise innovation, therefore limiting application of NPD models.

The Van de Ven et al (1999) study of innovation in the Minnesota Project found no support for such stage-based models. Correspondingly, Sharma (1999) suggests that reliance on milestone driven processes can constrain innovation development owing to strict and rigorous criteria. Conversely, many existing models are too vague (Van de Ven, 1993) limiting application to actual occurrences or routines. The organisational events in adopting change implicit in various types of innovation are also unaddressed.

There are several further criticisms that can be made about such new product development models and their relationship to the broader subject of innovation types. NPD models are dependent on the presumptions that commercialisation is the only innovation outcome and that product testing and R\&D divisions exist in organisations adopting such models. As such, NPD models were rejected for framing this study. The innate limitations in these models prevent an encompassing structure to facilitate understanding of the practical considerations in the journey. 
Rogers' Innovation Decision Process

Roger's studies on communication and innovation (for example Rogers and Singhal (1996) and Rogers' Diffusion of Innovations, in its fifth edition) were considered in attempting to identify a suitable model to which to ground this study. Rogers is considered the doyen of diffusion studies (Nutley et al., 2002). His seminal work on knowledge dispersal of an innovation led him to develop models synonymous with innovation diffusion research. Rogers (2003) describes diffusion as "the process by which an innovation is communicated through certain channels over time among the members of a social system" (p.5).

Rogers' Innovation Decision Process is recognised as one of the most frequently cited staged models (Nutley et al., 2002). It is a theoretical model containing five broad steps, the five steps outlining an individual's (or similar decision-making entity) progress when considering an innovation. The steps are:

1. Knowledge

2. Persuasion

3. Decision

4. Implementation

5. Confirmation.

The first step, Knowledge is exposure to the existence of an innovation and the understanding of it (Rogers, 2003). This occurs when an individual or organisation perceives a need, often arising from dissatisfaction or frustration. Likely adopters then need to be made aware of an innovation's presence in order to realise its benefits and implications (Zaltman et al., 1973).

Next, Persuasion refers to the forming of an opinion about the innovation, whether positive or negative. Specifically, it refers to the attitude towards the innovation rather than knowledge of it. Persuasion is not intended to induce a change in attitude compelled by a change agent, as is the usual definition of persuasion. Rather, this stage involves greater association with the innovation by others, leading to consideration of the implications of the innovation and the desire for further information in order to evaluate.

The first two steps can be considered jointly as socialising the innovation (Nonaka \& Takeuchi, 1995). According to Nonaka and Takeuchi (1995), the term Socialisation relates to the sharing of tacit knowledge held by one person to another. 
This is considered the embryonic step of testing an innovative idea with colleagues or a manager. Nonaka and Takeuchi (1995) go on to state that this step is followed by "amplification" (p. 84) through the company. This then is the converting of the tacit knowledge, the unshared concept held by the individual, to explicit, open knowledge with others in the company. A study by the Conseil de la recherché forestière du Québec (2004) agrees, relating the success of organisation innovation to new knowledge mastery, the managing of new knowledge to further organisational goals and collaboration. Achieving new knowledge mastery relies on interpersonal communication to develop organisational awareness and learning (Rogers \& Singhal, 1996; Savery, 2005) and leads into the next step in the process, the determining of the concept as worthy of further consideration.

Decision then is the third step, the engaging in activities that decide the future of an innovation. Rogers' model provides two clear options: either to adopt or reject the innovation. Rejection results in the termination of the innovation and completion of the journey. If the intention is to adopt, the innovation may be trialled for further refinement, or put into action through Implementation, the fourth stage. A component of the intention to adopt may be to request further information before a final decision is made. Rogers' advises that the Decision stage can be more difficult in a company situation than for an individual. This is due to the need to involve personnel not previously exposed to the innovation and potential resistance caused by reluctance to change or unsupportive organisational structure.

Implementation can involve re-invention, the modification or alteration of the innovation at this stage. Rogers' view is that such changes may be necessary to meet implementation or adopters' demands possibly eventuating in greater benefits or overcoming resistance. Van de Ven et al (1999) state that innovations cease when implemented, hence implementation is often the final stage in the innovation journey. Zaltman, Duncan and Holbeck (1973) agree, stating that at this stage the innovation has been legitimised by management translating it into a project, organisational or managed change in the organisation. The innovation's survival eventually results in it not being perceived as a separate entity, rather becoming a part of normal business function.

The fifth stage, Confirmation, may be necessary to reinforce or revise the decision, to reflect on the innovation's validity and value. It may mean termination of the innovation if there is evidence of conflicting information as to the innovation's 
benefits or there is disenchantment with the outcome. Rogers (2003) warns that during a company's progress through these five steps, an innovation may be abandoned at any time.

Rogers' model sets out the process which underlies dissemination of an innovation and its transformation to outcome value, yet the steps are nebulous allowing wide practical interpretation within each one. Williams (1999) advises that innovation systems cannot be planned in much detail nor adhere to rigid deadlines owing to the chaotic nature and unpredictability of innovation concepts, that a framework for accountability best be flexible, as offered by Rogers' model. As well, the model provides step by step logical guidelines in that, for instance, if step one is not undertaken lack of awareness would thwart an innovative idea from receiving due attention. Step two shifts from introducing the innovation at step one to convincing others of its merits. These are logical stages that a company may appreciate and hence lead to identify events that would correspond accordingly.

The Innovation-Decision Process model is considered a more appropriate theoretical framework for this study than Rogers' "Five Stages in Innovation Process in Organizations" (Rogers, 2003). This latter model starts with the premise of seeking innovations that fit with established company requirements rather than allowing for unplanned, informal or radical innovations. As well, the Five Stages model includes a step to reconfigure an innovation to fit a company's ideals. Although not refuting the relevance of this point, assuming the manipulating or limiting of an innovation in this way may distort the actual approaches developed by businesses.

Altogether, the Innovation Decision Process model provides a useful framework for the purpose of this study. It grants broad descriptions that enable a general precept to facilitate this study. The model lends flexibility whilst providing defined parameters that are not overly linear, nor pre-empting multiple lines of simultaneous activity. Rogers (2003) states that the steps provide a useful simplification of a multifarious reality, therefore assists to address concerns that the innovation journey be "messy and unpredictable" as described by Nutley et al (2002 p. 12).

Rogers' Innovation Diffusion Process (2003) model has been similarly employed in prior studies to underpin research. For instance, Weitzel and 
Hallahan's (2003) study on organisational adoption of an intranet-based reporting system used the model to provide structure for exploring the success of innovation adoption. The model was also used by Savery (2005) to further knowledge on the issues relating to adopting innovation by public relations practitioners. Furthermore, Woodside and Biemans (2005) employed the model in reviewing the innovation decision process in an organisational context.

\section{Managing Innovation Features and Practices}

Effective realisation of innovation within an organisation and the competitive advantages offered by the successful unlocking of innovation value is most likely interrelated to a company's structure, culture and environment. Senge (1992) introduced the concept of the learning organisation to enable companies to build organisational and individual skills in order to respond and drive rapid change and so thrive in the competitive business environment. Some of his key precepts include flexibility, adaptability, receptivity to ideas from all sources and shared team development, a view shared by Kotter (1996) in proposing how a $21^{\text {st }}$ century company will appear. Nemeth (1997) (cited in Katz (2004)) argues that flexibility and openness together with dissension underlie innovation encouragement. Jones (2002b) talks about "a supportive and motivating environment" (p. 111) as necessary to facilitate idea creation in an organisation. Jones (2002b) elaborates (citing 3M's innovation traditions) presenting empowerment, communication and recognition with vision as drivers of continuous improvement. Further, Jones contends that an open environment, orientated to respecting and enabling individuals and a flat organisational structure are critical to support innovation in a company.

Williams (1999) focuses on the need to recognise innovation generation, rewarding initiatives financially or additional time off. He also proposes softer reward methods such as praise, opportunities to act further with innovations and greater self-direction. Jones (2002a) agrees with Williams (1999) that open communication and open mindedness are necessary features to support continual sharing of concepts between employees, up and down status levels and across all boundaries.

The Boston Consulting Group's Innovation survey (2005) takes a slightly different but complementary approach. They recommend alignment as the focus of the innovative company, centring on three key areas: people, identifying and supporting collaboration; secondly, environment, demonstrating by management example that innovation is paramount; finally, measuring and rewarding innovation 
activity. Altogether these various features portray an image of what is required to be present in a company in order to successfully realise innovation. Conversely, bureaucracy, rigid hierarchy, change resistance and limited communication affect the ability of a company to succeed in unlocking innovation value (Boston Consulting Group, 2005; Srivastava \& Gnyawali, 2006).

\section{Sources of Innovation}

Another practice to effectively managing innovation is the welcoming of ideas from all sources (Senge, 1992). As shown in Figure 2 (p. 15), innovation can be the outcome of intentional planning or it may eventuate from unexpected agents (for example employees, customers). The view that innovative ideas come from throughout organisations is supported, in particular by Zien \& Buckler (2004) and their Polaroid Invention and Innovation Research Project. The Polaroid model (Figure 4) exemplifies the numerous sources of ideas that lead to innovation. Statistics NZ (2004) also lists multiple origins of innovation concepts both internal and external to an organisation.

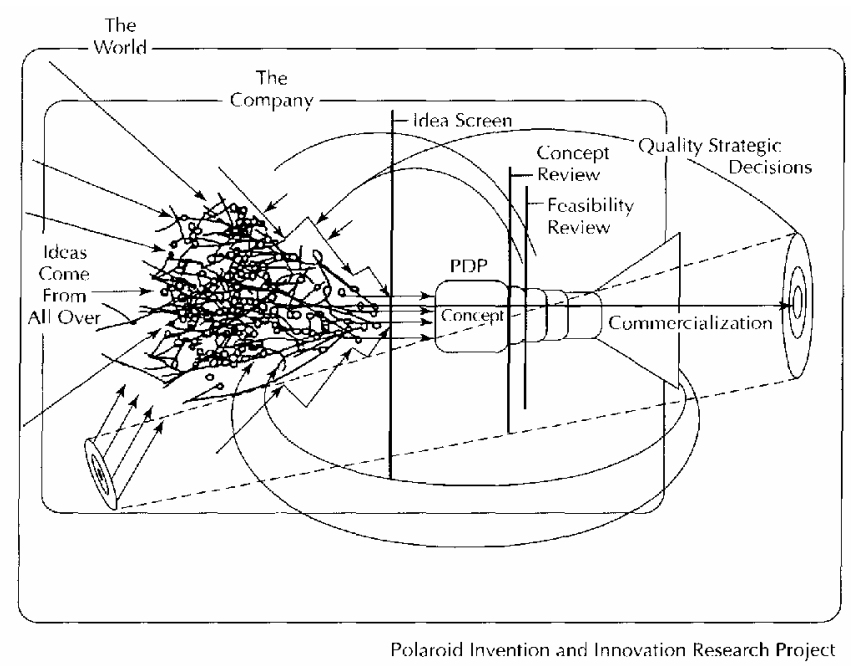

Figure 4: Sources of innovation. (Zien \& Buckler, 2004 p. 481)

Often innovative ideas are serendipitous without planned intervention or as expected outputs by management or research and development (R\&D) departments (Birkinshaw et al., 2005). That sources are numerous, coming from both expected and unexpected origins reinforce the premise that generating ideas for innovation is not the hurdle to organisations. Hence, depending on systems that focus on planned innovations can be constrictive, overlooking haphazard sources of innovative ideas. 


\section{Innovation Reviews}

Reviewing innovations once implemented may assist to improve the success of innovation realisation. Rogers' (2002) third and fourth steps of Implementation and Decision and the fifth step, Confirmation perform the task of assessing the use of resources and value output in realising an innovation. Similarly, Cooper's Stage Gate process (2005b) uses checkpoints to review, ensure viability and assist best selection for resource application. Tidd and Hull (2003) claim that a review process assists outcome with reviews of innovations and innovation systems also suggestions of Pinchot and Pellman (1999). Alternatively, they suggest that if the innovation is no longer desirable, it is abandoned.

\section{Provisional Steps in the Innovation Journey}

Various authors do provided some indications of potential steps in a practical innovation-to-outcome system. A structured process assists identifying the best mix of innovations to meet long term goals and to help ensure resources are allocated correctly (Pinchot \& Pellman, 1999). Suggestions as to functional occurrences in the structured process are proposed firstly by Hamel (2000) and Kanter (1982). Hamel (2000) suggests initial steps as selling the idea to the line manager, preparing a business concept, finding a sponsor, building support. Kanter (1982) broadly outlines the innovative practice as three phases: definition of the project, building support and action.

Pinchot and Pellman (1999) give overarching indicators of stages (Table 1, supported by other authors). The seminal work of Chase $(1978 ; 1991)$ on service organisations (cited in Tidd \& Hull (2003) suggests that the basic steps are similar in service industries as well as manufactured goods companies, although differing in execution. Chase $(1978,1991)$ lists the basic steps as generating innovations, developing the concepts, building and implementing the ideas. He posits that the names may change but the four phases are consistent. Although helpful these system stage indicators are referred superficially and incompletely, falling short of identifying realistic practical events a company could employ. 
Table 1: Potential stages in the innovation-to-outcome journey

(source: Pinchot and Pellman (1999) and supported by other authors)

1. Definition of project (Annacchino, 2003; Kanter, 1982).

2. Selling the idea to reporting manager (Cooper \& Edgett, 2001; Hamel, 2000).

3. Prepare a business concept (Annacchino, 2003; Cooper \& Edgett, 2001; Hamel, 2000).

4. Find a sponsor (Hamel, 2000).

5. Build support (Hamel, 2000; Kanter, 1982).

6. Action (Kanter, 1982).

\section{Influences on the Innovative Journey: Barriers and Supports}

The effectiveness of achieving a successful innovation outcome depends on what is experienced during the internal journey. Some activities support value realisation while others thwart the unlocking of innovation value. Yin (2004) agrees, stating that "uncertainties in the implementation process may lead innovations to fail or succeed"(p.100). Lacking direct indicators, analysis of literature on developing innovative organisations helps to identify barriers that may be transferred to the innovation journey. Similarly, supports to the innovative organisation could also be translated as facilitators to realising innovation outcome, as subscribing to similar tenets. Cooper (1998) recommends taking care to identify innovation implementation barriers when the objective is success of outcome. In other words, the same variables impacting on companies desiring to increase innovation capability may also influence the innovation system (McFadzean et al., 2005).

The Commission of the European Communities (2003) perceives obstacles to innovation as being created by resistance to change and the challenge to existing procedures. Tidd and Hull (2003) agree, stating that risk-avoiding behaviour is normal in companies. Christensen and Raynor (2003) express the view that innovations fail because managers or organisations are inept or organisational capabilities are insufficient to capture the value. Christensen (2005) cites concerns of fitting innovative activities into daily routines without efficiency being compromised. These studies focus on barriers to achieving an innovative organisation hence indicate impediments to unlocking innovation value.

The influences on the innovation journey can be summarised under the capabilities triptych proposed by Christensen and Raynor (2003) as: 
- Resources to succeed (tangibles: assets, people, things);

- $\quad$ Processes that assist (interaction, coordination, communication);

- Values that recognise and balance resources between new and

established demands (criteria for decision making, culture).

In their book "Seeing What's Next" Christensen, Anthony and Roth (2004) propose that these three areas are key to successfully addressing opportunities. Summarising influences on the innovation journey in this way provides guidance to which area a support or barrier impacts (see Table 2 p. 28 and Table 3, p.30).

\section{Resources.}

Commitment of scarce resources and access to adequate funds are cited as fundamental to corporate innovation by Jauhari (2001) and Sharma (1999). Amabile's (1998) extensive work on creativity in business refers to the main resource decisions (time and money) as major influences on success or failure. Resentment towards the innovation can manifest itself through the loss of finance from the corporate's core operations, distraction of key staff (Day, Mang, Richter, \& Roberts, 2001) and relates to senior management's competencies and behaviour determining resource allocation (Pavitt, 2003).

People may be considered both instigators and facilitators of innovation whilst also inhibiting or preventing innovation (Van de Ven et al., 1999) if innovation resistant. Leadership is paramount, Van de Ven et al (1999) stipulates, to overcome resistance and manage the human impact on innovation. The paradoxical loop of co-operation and competition for resources and personnel involvement demands pluralistic leadership: combining diverse perspectives to address the ambiguity and uncertainty of innovation.

\section{Processes.}

Pavitt (2003) states that innovation progress depends on cross-functional collaboration between departments. Lack of this cross-functionality can be a barrier, which can be further compounded by the rigidity of an organisation (Srivastava \& Gnyawali, 2006). As well innovation is required to navigate through the bureaucratic maze (2002; Kanter, 1982; Kuratko \& Hornsby, 2001; Mclntyre, 2001; Sharma, 1999). Organisational rigidity and bureaucracy are both anathemas to innovation. Christensen (2005) agrees, advising that simply being part of a large organisation brings bureaucratic obstacles. 
Values.

Corporate values and the business model are advocated by Chesborough and Rosenbloom (2002) as major influences on the innovative organisation. Amabile (1998) contends that management negativity has severe consequences, often damaging the innovation outcome. Fielden and Malcolm (2006) add that management is less flexible in large companies, while Van de Ven (1993) stipulates that relaxation of managerial control may be necessary to provide the necessary conditions to support innovation activity. Values that drive decision criteria result in the decision process often being political, promoting self interest rather than a balanced measure of the risks, benefits and costs (McIntyre, 2001; Pavitt, 2003). Whilst Birkenshaw et al (2005) refer to the "corridors of comparative indifference" (p31) throughout organisations hindering innovation.

\section{Tabulation of Barriers.}

A summary of potential obstacles, extrapolated from literature on the innovative organisation (see Table 2, p. 28) is extensive. The list provides ample evidence of the difficulties to overcome in realising innovation outcome. Of significance to New Zealand companies, Statistics New Zealand (2004) found that the majority of companies cited lack of management resources (56 percent), cost of developing innovations together with insufficient capital (53 percent) and lack of appropriate staff (51 percent) as dominating barriers hampering innovation.

\section{Enablers of the Innovation Journey}

Conversely, Table 3 (p. 30) lists facilitators distilled from literature on the innovative organisation indicating potential supports to the innovation journey. Scholars such as McIntyre (2001) and Kanter (1982) complement other authors' findings (Christensen, 2005; McLaughlin, Baessant, \& Smart, 2005; Van de Ven, 1993) and are listed in Table 3. Christensen (2005) and Van de Ven (1993) contend that despite the bureaucratic nature of large companies, they often have more innovation flexibility and choice. Larger companies most likely enjoy greater financial support and sources of ideas to support innovation activity. Success is attributed to corporate culture and a business model supportive of innovation (Kaplan \& Norton, 2000; VanGundy, 2005). Yet the type of company is irrelevant according to the Boston Consulting Group (2005) survey that found all types of organisational structures are present in successful innovative companies. 


\begin{tabular}{l|l}
\hline Barriers to the process & Reference \\
\hline
\end{tabular}

\section{Resources}

Competing management priorities due to finite resources/resource constraints.

Mis-allocation of (limited) investment finances.

(Bannon \& Grudin, 1990; Bean \& Radford, 2002; Christensen, 1997; Cooper \& Edgett, 2001; Council on Competitiveness, 2005;

Pinchot, 1985; Sharma, 1999; Srivastava \& Gnyawali, 2006; Tidd \& Hull, 2003; VanGundy, 2005)

Rewards and incentives unsuitable or limited in recognising innovation. Encouraging status quo; "Turf protection", protecting existing practises.

(Batiz-Lazo \& Woldesenbet, 2006a; Bean \& Radford, 2002; Commission of the European Communities, 2003; Knight, 1987; Kuratko \& Hornsby, 2001; Van de Ven \& Engleman, 2004; VanGundy, 2005)

(Christensen \& Raynor, 2003)

Management's inability to refuse a concept or identify best choice tying up critical resources better allocated to other innovative projects.

"Bandwagon" effect: where too many people want to be part of the innovation activity.

Fear that innovation will cannibalise existing products.
(Knight, 1987)

(Batiz-Lazo \& Woldesenbet, 2006a; Christensen, 1997)

\section{Processes}

Management inability to implement innovation, middle and even senior managers who do not understand that the company wants and needs innovation.

Processes that do not support change, including lack of cross-functionality structures.

Innovation introducing contradictions, managers needing to appease the need for hierarchy whilst encouraging creative anarchy; control versus flexibility, continuity among chaos.

Decision making "by consensus".

Multiplicity of meetings to decide merit or to evaluate.
(Amabile, 1998; Batiz-Lazo \& Woldesenbet, 2006a; Bean \& Radford, 2002; Kanter, 2002)

(Ahonen, 2005; Amabile, 1998; Bean \& Radford, 2002; Kanter, 2002; Sharma, 1999; Van de Ven \& Engleman, 2004)

(Bean \& Radford, 2002; Maidique \& Hayes, 1998; Pinchot, 1987)

(Amabile, 1998; Bannon \& Grudin, 1990; Kanter, 2002)

\section{Values}

Preoccupation with current activities and markets.

(Bannon \& Grudin, 1990; Bean \& 


\begin{tabular}{l|l}
\hline & $\begin{array}{l}\text { Radford, 2002; Garvin \& } \\
\text { Levesque, 2004) }\end{array}$ \\
\hline $\begin{array}{l}\text { Short term focus and/or an excessively internal } \\
\text { focus. }\end{array}$ & $\begin{array}{l}\text { (Council on Competitiveness, } \\
\text { 2005; Hamel \& Getz, 2004; } \\
\text { Mclntyre, 2001) }\end{array}$ \\
\hline $\begin{array}{l}\text { (Inappropriate) Strategic orientation. } \\
\text { (Batiz-Lazo \& Woldesenbet, } \\
\text { 2006b) }\end{array}$ \\
\hline $\begin{array}{l}\text { Internal politics. } \\
\text { Organisational resistance to change and/or } \\
\text { cultural inertia, satisfaction with status quo or } \\
\text { guarding territories (hostile bureaucracy). } \\
\text { Lack of support to adopt change. }\end{array}$ & $\begin{array}{l}\text { (Batiz-Lazo \& Woldesenbet, } \\
\text { 2006a; Bean \& Radford, 2002; } \\
\text { Kanter, 1982, 2002; Kuratko \& } \\
\text { Hornsby, 2001; Mclntyre, 2001; } \\
\text { Srivastava \& Gnyawali, 2006; Van } \\
\text { de Ven, 1986; Van de Ven \& } \\
\text { Engleman, 2004) }\end{array}$ \\
\hline
\end{tabular}


Table 3: $\quad$ Factors that facilitate the innovation journey (supporting realisation)

\begin{tabular}{|c|c|}
\hline Enablers & Reference \\
\hline \multicolumn{2}{|l|}{ Resources } \\
\hline $\begin{array}{l}\text { Having sufficient resources to support } \\
\text { innovations. }\end{array}$ & (Van de Ven, 1993) \\
\hline $\begin{array}{l}\text { Access to resources to develop innovative ideas } \\
\text { not requiring senior level approval. }\end{array}$ & (Mclntyre, 2001) \\
\hline $\begin{array}{l}\text { Rewarding successful innovation (cash bonuses } \\
\text { and other incentives). }\end{array}$ & (Mclntyre, 2001) \\
\hline $\begin{array}{l}\text { Overarching or multi-disciplinary team to drive } \\
\text { process. }\end{array}$ & $\begin{array}{l}\text { (Christensen \& Raynor, 2003; } \\
\text { Khurana \& Rosenthal, 2002; Tidd } \\
\text { \& Hull, 2003) }\end{array}$ \\
\hline $\begin{array}{l}\text { Having a champion or ambassador in } \\
\text { management to support and drive the process. }\end{array}$ & $\begin{array}{l}\text { (Garvin \& Levesque, 2004; } \\
\text { (Khurana \& Rosenthal, 2002; } \\
\text { Knight, 1987; McAdam, 2005; } \\
\text { Nonaka \& Takeuchi, 1995; } \\
\text { Pinchot \& Pellman, 1999; Roberts } \\
\text { \& Fusfeld, 2004; Rogers, 2003; } \\
\text { Sharma, 1999; Tidd \& Hull, 2003; } \\
\text { Van de Ven et al., 1999) }\end{array}$ \\
\hline $\begin{array}{l}\text { Training of staff to be more innovative or } \\
\text { responsive to innovation. }\end{array}$ & $\begin{array}{l}\text { (Commission of the European } \\
\text { Communities, 2003; McAdam et } \\
\text { al., 2000; Statistics New Zealand, } \\
\text { 2004) }\end{array}$ \\
\hline
\end{tabular}

\section{Processes}

\begin{tabular}{l|l}
\hline $\begin{array}{l}\text { Drawing on customers' experience to generate } \\
\text { new ideas. }\end{array}$ & $\begin{array}{l}\text { (Mclntyre, 2001; Zien \& Buckler, } \\
2004)\end{array}$ \\
\hline $\begin{array}{l}\text { Interrelationship with the customer by } \\
\text { "backroom" personnel. }\end{array}$ & (Mclntyre, 2001) \\
\hline $\begin{array}{l}\text { Free flow of information, resources crossing } \\
\text { departmental boundaries and hierarchy. }\end{array}$ & $\begin{array}{l}\text { (Kanter, 1982; Mclntyre, 2001; } \\
\text { Pinchot \& Pellman, 1999; Zien \& } \\
\text { Buckler, 2004) }\end{array}$ \\
\hline $\begin{array}{l}\text { Protecting innovation from bureaucratic } \\
\text { limitations. }\end{array}$ & (Hesselbein et al., 2002). \\
\hline
\end{tabular}

\section{Values}

Setting goals for innovative achievement (for example: linking results with sales strategy).

Encouraging a long-term perspective, linking with clarity of innovation strategy.

(Maidique \& Hayes, 1998; Mclntyre, 2001)

(Kanter, 1982; Maidique \& Hayes, 1998; Mclntyre, 2001; Pinchot \& Pellman, 1999)

Entrepreneurial and management culture,

(Birkinshaw et al., 2005; 


\begin{tabular}{l|l}
\hline $\begin{array}{l}\text { supportive of innovation vision, openness to new } \\
\text { ideas, open communication. Innovation framed } \\
\text { as positive. }\end{array}$ & $\begin{array}{l}\text { Christensen, 2005; Maidique \& } \\
\text { Hayes, 1998; Sathe, 2003; Tidd et } \\
\text { al., 1998; Van de Ven, 1986, } \\
\text { 1993) }\end{array}$ \\
\hline $\begin{array}{l}\text { Cultural pride in company's (innovative) } \\
\text { achievements, cohesion. }\end{array}$ & $\begin{array}{l}\text { (McLaughlin et al., 2005; Zien \& } \\
\text { Buckler, 2004) }\end{array}$ \\
\hline Company/individual propensity for innovation & (Woodside \& Biemans, 2005) \\
\hline $\begin{array}{l}\text { Accepting failure (overcoming the fear of } \\
\text { mistakes in being creative, being comfortable } \\
\text { with change and conflict). }\end{array}$ & $\begin{array}{l}\text { (Maidique \& Hayes, 1998; } \\
\text { Mclntyre, 2001; McLaughlin et al., } \\
\text { 2005; Pinchot \& Pellman, 1999) }\end{array}$ \\
\hline $\begin{array}{l}\text { Organisational structure and cross functionality } \\
\text { of divisions. }\end{array}$ & $\begin{array}{l}\text { (Christensen \& Raynor, 2003; } \\
\text { Sheppard \& Canning, 2006; Tidd } \\
\text { et al., 1998) }\end{array}$ \\
\hline $\begin{array}{l}\text { Relevance or advantage of innovation to } \\
\text { individual perspective. }\end{array}$ & \begin{tabular}{l} 
(Woodside \& Biemans, 2005) \\
\hline $\begin{array}{l}\text { Innovation introduced as analogous and } \\
\text { complementary to previous successful } \\
\text { innovations. }\end{array}$
\end{tabular} \\
\hline $\begin{array}{l}\text { Participative or team style of management } \\
\text { supporting subordinates. }\end{array}$ & $\begin{array}{l}\text { (Birkinshaw et al., 2005; Van de } \\
\text { Ven, 1993) }\end{array}$ \\
\hline
\end{tabular}


Referring to the list of supports in Table 3 some enablers dominate the innovation journey. In particular, that a champion is necessary for demanding or complex innovations. This is someone prepared to visibly and vocally support an innovation through to realisation and is often (but not always) someone in management. The involvement of senior employees is often necessary to innovation outcome success as these people possess the necessary credibility and access to resources.

Likewise, a company having a culture receptive to innovation and encouraging of innovation activity is a crucial support to outcome and value realisation. Such organisation cultures support change and creative endeavours, for example reviewing incumbent activities to improve offering. Interestingly, the list indicates the greatest single area of support is contained in company values, intangible but imperative facilitators to unlocking innovations.

In comparing the various innovation supports with barriers it appears that what constitutes a support is often the reversal of a barrier. For instance, management as a barrier may thwart the outcome process through change resistance or misalignment. However, in turn management becomes a critical support when championing an innovation. Another example is the pivotal position of senior managers. Executives have an awareness of overall company activities which helps them realise the benefits of a proposed innovation and therefore the desire to realise its value. Yet these same employees are generally the ones to evaluate the best use of scarce resources. Refusing some innovations the necessary support therefore prevents their value being realised (Van de Ven et al., 1999).

In conclusion, the literature confirms that innovation is paramount to businesses' survival and company growth. Yet the unlocking of innovation value is dependent upon the success of the internal innovation-to-outcome process, a process that currently lacks empirical practical definition. Prescriptive understanding of how to realise innovation value remains unclear. Extant literature focuses on the innovative organisation or offers abstract concepts rather than practical steps for processing innovation through an organisation. 
This paper contends that parallels can be drawn between the process of strategic planning, (which has been successfully formalised) and the innovation-tooutcome journey. Both are intrinsically idiosyncratic in nature and highly individual to a company, yet critical to continuing success. Being able to map strategy lends support to mapping this internal innovation system. Mapping then assisting companies to realise greater success through capturing the value of innovations and is aided by maximising enablers and limiting hindrances to the process.

The influences (barriers and supports) on the innovation journey are extrapolated from literature on developing the innovation capable organisation as providing parallels to the internal innovation journey. Details on functional events in the innovation-to-outcome process appear to be lacking in empirical writings with some exceptions, specifically New Product Development models. Although existing models including NPD offer some guidance in realising the outcome, the scholarly approach or myopic focus restricts application to understanding the practical innovation journey and workable solutions to unlocking innovation value.

That innovation inherently brings change provides further substance to the need to manage this critical process. Introducing change can often meet resistance in an organisation. Realising a clear innovation system strategy could support businesses to overcome such limitations and achieve greater innovation outcome effectiveness. Therefore, this study draws on Rogers' (2003) Innovation Diffusion Process model for considering the events of the innovation journey. The model is employed as the underlying premise to this study's research, discussed further in the next chapter. 


\section{Chapter 3 \\ METHODOLOGY}

\section{Chapter Outline}

The previous chapter considered existent literature on innovation activity in business and the journey of innovation within a company. This section firstly revisits the research question and objectives. Next, the chapter outlines the reasoning for a qualitative approach using inductive logic. The rationale for selecting multiple case studies is outlined together with the advantages and disadvantages of this approach. Next, justification for the choice of industry and the three companies is presented leading into details on data collection and the data analysis process.

\section{Research Objectives}

The review of literature illuminated various conceptual models of innovation systems providing a conjectural basis for understanding the journey. However, these theoretical constructs (Van de Van (1999) and Schroeder (1986)) lack practicality. The literature appears deficient in models that provide functional instructions that companies can adopt to support success in realising innovation outcomes. Aiming to address this gap, the key question this research will respond to is: "How can the process of managing innovation from inception to outcome be modelled?" together with the objectives:

1. To model the path of innovation through the event sequence from inception to completion or abandonment.

2. To identify from examination of these innovation models any patterns or common practices that typify the management of transforming innovation.

3. To determine any patterns and practices hindering the process reaching a successful outcome.

4. To determine any patterns and practices associated with successful, value-creating outcomes.

5. To develop a framework that will provide useful as a basis for guiding best practice in innovation management

\section{Interpretative Qualitative Approach}

The gathering of qualitative data was indicated in order to address the above gap in realising innovation outcome in business. Collis and Hussey (2003) state that 
when investigating human behaviour a qualitative research method can be employed to enable gathering of descriptive details, a viewpoint shared by Thomas (2003). Kohlbacher (2005) goes further in stating that "only qualitative methods are sensitive enough to allow the detailed analysis of change" (p. 9). In addition, she states that the qualitative paradigm "is less likely to impose restrictive a priori classifications" ( $p$. 9) on data collection. The indication is that qualitative methods are advantageously used when the phenomenon is not well known or understood with the aim of generating new theories, therefore appropriate to this study.

Further, Morse and Richards (2002) explain that the interpretive, qualitative approach provides methods to support understanding of what is likely to be found, when current information appears inadequate. Similarly, if there is "a concern to understand the world as it is... within the frame of reference of the participant" (Van de Ven \& Rogers, 1988, p.7). Likewise, a qualitative study, as Marshal and Rossman (1999) posit, provides "quality, depth and richness in the findings"; and "a thick description" (p.16) necessary to gather promising data on the subject studied. The use of a quantitative approach was rejected as collection of statistical and numerical data would not provide the critical descriptive detail required for this study.

An objective of this study is to construct a map of the journey through dissemination of themes and evidential patterns from data. Therefore data are required to be descriptive and subjective (Collis \& Hussey, 2003) reinforcing the selection of a qualitative research method. Further, inductive logic was indicated (Somekh \& Lewin, 2005) as examining and analysis of the phenomena leads to patterns which then leads to formulating theory (Cavana, Delahaye, \& Sekaran, 2000). Inductive logic seeks to find meanings from empirical data (Somekh \& Lewin, 2005) with themes and patterns emerging from the data (Patton, 1980). This is in contrast to deductive thinking which starts with theory to develop hypothesis, using findings to reject or support theory.

Additionally, an analytical approach was necessary to develop understanding of a company's innovation-to-outcome journey, going beyond simply describing characteristics (Collis \& Hussey, 2003) to explain the why of the internal innovation process. The focus in explanatory research is on questions that ask why and considers causal implications. This is a necessary step when identifying the variables consistent within the innovation journey and possible indicators of best practise. The why in this instance relating to the steps in the system and why of 
events and activities employed. Yin (2003a; 2003b) recommends case study as ideally suited to addressing why questions, as does Kohlbacher (2005).

\section{Theoretical Framework}

Yin (2003b) and Marshall and Rossman (1999) stipulate that qualitative studies are best embedded in suitable theoretical domains as an established model gives greater reliability and replicability. Van de Ven and Rogers (1988) expand on this premise proposing that theory is not absent when commencing with data. Rather, that it has another role. Theory, in this situation, defines the objectives of interest and guides the propositions from subjective meanings. Consequently, drawing on Rogers' (2003) Innovation Decision Process grounds the data in a theoretical framework which then supports analysis. Additionally, a conceptual framework is critical (Peel, 2003) to further research rigour. This was developed through the study's literature review and in considering existing business innovation practices as espoused by the academics cited herein.

\section{Election of Case Study as Research Strategy}

Considering the above, the use of case study as a strategy (not a method as Kohlbacher (2005), de Vaus (2001) and Yin (2003b) observe) is appropriate as various methods can be applied to achieve a wealth of description (Somekh \& Lewin, 2005) whilst investigating the phenomena of contemporary events. The events being researched in real-life, real-time context within their natural settings (Collis \& Hussey, 2003; McLaughlin et al., 2005; Yin, 2003a). Employing the case study approach in this instance facilitates understanding of what actually happens by examining companies' currently occurring internal innovation systems.

Social science research uses case studies for organisational studies (Kohlbacher, 2005) as they enable a better understanding of (company) culture and values: the influence of less tangible factors. Two-fold strengths exist in the case study method. Firstly, triangulation of data collection; secondly, this is complemented by the use of multiple analysis methods. These assist reliability and internal and construct validity (Yin, 2003a). Additionally, in this situation, the case study design was indicated as explanatory, aptly suiting this study's subject as seeking to achieve practicable explanations of the innovation journey phenomena.

Yin (2004) proposes that a case study is particularly suited when phenomenon and content are not able to be separated, especially relevant in attempting to 
distinguish a company's actions in relation to innovation realisation. In addition, he advises that case studies help to address the why and how of occurrences using a range of empirical tools, resulting in an explanatory case study approach. Eisenhardt (1989) stipulates that cases can be used to generate theory, Wilson (2005) develops this, proposing that cases provide stories that then permit frameworks and theories to emerge from data gathered, as is the aim of this study.

A multiple case study design was thus employed, a variant of the case study approach (Yin, 2003b). Yin (2003b), whose seminal work on case studies is drawn on extensively in this study, argues that multiple case studies are more robust than single-case studies. However, he recommends that participants are selected to maximise theoretical replication, occurring when contrary results are gathered, but for expected reasons. Or literal replication achieved through similar results being realised. Tellis (1997) reinforces the need for case selection to follow replication logic, not sampling logic, iterating consistency in selection. By selecting multiple companies from one sector, ones that can be regarded as typical of their type, helps to provide a strong basis for replication.

For the purpose of this research, electing to study more than one case permitted a comparison of the various internal innovation-to-outcome systems developed by the subject firms and provide a more rigorous result (Miles \& Huberman, 1994; Yin, 2004). A multi-case study facilitates such comparative study (Jankowicz, 1992) providing perspective (Hamel, Dufour, \& Fortin, 1993) and the opportunity to systematically explore the different systems, or the similarities thereof. This furnished a greater foundation for the recognition of patterns during analysis and interpretation of results (Bouma, 2000).

Tellis (1997) and Hamel, Dufour and Fortin (1993) comment that the goal of case studies should be to establish parameters which then can be applied to other situations. Yin (2003a) agrees with this sentiment, stating that case studies are not samples but are used to expand concepts. Tellis (1997) goes on to state that multiple cases "strengthen the results by replicating the pattern matching, thus increasing confidence in the robustness of the theory" (p. 4). 
Comparative Uses of the Case Study Approach

Marshall and Rossman (1999) advocate case study method as having a fertile tradition in research of organisations, providing details and depth. Yin's "Case Study Anthology" (2004) includes a variety of seminal single and multiple case study examples. These demonstrate best practice in case study method and provide abundant detail on case study technique and applicability, information which was drawn from in this study.

Numerous other researchers have used case study strategy. For instance, Von Stamm (2003) uses mini-cases throughout her book to reinforce relevance of creativity and innovation in organisations providing insights into embedded practices. Additionally, Schroder et al (1986) employs case studies extensively in their analysis of innovation processes in business. Another example of the case study approach being employed in innovation studies is Sharma's (1999) nine company case study. Sharma identified patterns of innovation implementation dilemmas in large companies, comparing multiple cases from which results were extrapolated.

Batonda and Perry (2001) drew on case study methodology as appropriate for studying the contemporary issue of relationship development processes within firm networks. Additionally, Govindarajan and Trimble (2005) uses the method to identify barriers to building innovative ventures within companies. Finally, Van de Ven et al (1999) utilises case study strategy in comparing three companies' organisational structures in relation to the innovation journey. Therefore, the choice of case study for this research project follows previous research into innovation in business.

\section{Interviews}

Explicit data were gathered using semi-structured interviews conducted with key personnel involved with innovation activity in each company. Considered an important source of information for case studies (Tellis, 1997; Umit, 2005), Yin (2004) advocates open-ended interviews as a frequent form of gathering evidence in case studies. These interviews are more often in the mode of conversation, which was engaged in this study. Keats (2000) advocates interviews as suitable when it is desirable to know what is being thought by people and if the ideas are intricate or problematic to express.

Wengraf (2001) and Jankowicz (1992) state that semi-structured interviews assist when the story related to a participant's experience is desired. As the 
literature review has indicated, for instance, Van de Ven et al's studies (1999) and Pavitt's (2003) propositions, the journey tends to be informal and undefined. Hence, explanation of currently employed methods required close examination with the individuals involved seeking their opinions and experience with each company's process.

\section{Concerns with Method Selection}

Rudestam and Newton (2001), use the term trustworthiness to represent the various important facets of empirical research. When designing this study the four tests proposed by Yin (2003b) to address short comings of research and of case study approach in particular were closely considered. Yin (2003b) details these tests as construct validity, internal validity, external validity and reliability. However note was also taken of Hamel et al (1993) contention that some criticisms of case study strategy are historically a negative critique of sociology as a discipline rather than of case studies per se. In other words opponents of case study strategy are actually arguing the conflict of methodology choice (Tellis, 1997) between quantitative and qualitative based research.

\section{Validity}

Validity refers to research findings actually providing legitimate information to give accurate indicators (Collis \& Hussey, 2003). Several measures of validity are relevant to this research. Firstly, construct validity - which is considered particularly problematic in case studies (Tellis, 1997; Umit, 2005; Yin, 2003b) - is the ability to ensure that what is being measured is the innovation journey resulting from the implemented research strategy; the validity of construct choice to measure what it is to measure. In other words, whether the case study strategy uses the correct measures and provides evidence of companies' internal innovation-to-outcome systems. In this situation the proposed three remedies by Yin (2003a) were employed: the use of multiple sources of evidence (for example interviews, public and company information) together with an established chain of this evidence and; thirdly, the review of the draft report by key participants. All provided support that what was being measured was achieved via the research methodology employed.

\section{External validity}

Construct validity and external validity both relate to the ability to generalise. However, with external validity it is the extrapolating of findings of the study to other situations and companies, or transferability (Marshall \& Rossman, 1999). Criticisms 
of case study strategy include the argument that conclusions cannot be generalised. Or to put it another way, that findings are not able to be transferred or extended to larger population or to assist understanding wider phenomena. This premise is due to any particular case study under analysis being considered unique and so reducing reliability (Thomas, 2003). In this context the constraint is addressed through studies of multiple entities (Miles \& Huberman, 1994; Yin, 2003b) identifying similarities and differences, drawing on findings to create a generalised argument and identified patterns. Apropos of the above, the aim here is to select cases that prove or provide parameters that are then able to be related to other circumstances. The constraint is also addressed through using replication logic in selecting multiple case studies (Yin, 2003b).

Using Rogers' (2003) model (p. 19) as the theoretical framework further supported external validity. Yin (2003) proposes that having a framework provides a conduit giving parameters and permitting generalisation to new instances. It can also boost the potential of findings for further applicability, guiding data collection and analysis (Yin, 2004).

\section{Internal Validity}

As this study is explanatory, internal validity is a concern (Eisenhardt, 1989; Tellis, 1997). This is the making of inferences regarding cause and effect, that companies' innovation systems are as described and do provide outcomes of value to the firms. Additionally, Yin (2003b) states that inferences by the investigator can be suspect. To overcome these, use of multiple sources of evidence assisted as did interviewing several individuals at each site providing a range of experiences within each case. Linking data gained from multiple interviews within a company using the same questionnaire provided information that could then be matched to the theoretical framework, additionally drawing on external sources to validate claims of innovation success. De Vaus (de Vaus, 2001) stipulates that explanatory case studies can achieve strong internal validity by developing an extensive casual account comparing and assessing causalities to provide a balanced report. Both internal and external validity were further assisted by triangulating data (Eisenhardt, 1989).

\section{Reliability}

A problem with qualitative studies is considered to be reliability owing to the inability of the researcher to control the environment in which the phenomena is 
being studied (Yin, 2003b) . Reliability was achieved, in particular, by the development of case study protocol (Tellis, 1997; Yin, 2003b). Tellis (1997) advises that a typical protocol includes an overview of the research project, details on research method and application, the objectives of the study and report content compositions. These have been developed throughout this study in order to facilitate reliability and replicability.

\section{Replication}

Replication (as with external validity) is the ability to replicate the study. In this case, replication is promoted through the provision of Rogers' (2003) model as the theoretical framework grounding the study. In addition, the evidence chain and protocol supports replication. For instance, the interviews were based on a standard format written down to guide the interviewer, guide the process and to provide consistency. Replication is also assisted by an audit trail (Rudestam \& Newton, 2001) the keeping of thorough records of the entire study to afford management and clarity of process and was employed in this study.

\section{Interviewing}

As interviews were the main method of gathering data, the constraints to this activity are also considered. Bouma (2000) warns that interviewing has inherent concerns being reliant on cooperation and willingness by participants to discuss the subject. Another concern is that interviews are dependent on a rapport being established between the parties to facilitate cooperation (Marshall \& Rossman, 1999). Moreover, the variability of data from interviews being misrepresented or misconstrued (Collis \& Hussey, 2003) with the subjectivity of the interviewer potentially influencing data analysis - subjectivity additionally being a concern with qualitative methods.

These concerns were addressed through the checking and rechecking of data, providing a draft report to case study participants to ensure the interpretation of data collected was correct and to minimise errors (Bouma, 2000). Iterative visits to primary data during analysis also assisted in examining plausible explanations (Marshall \& Rossman, 1999). As well, some questions were repeated in a different format during the interview (Keats, 2000) as checks to consistency of responses. 


\section{Selection of Industry and Case Study Companies}

Different industry sectors provide differing environments in which to discuss innovation. The selection of companies within one sector provided an industry setting which assisted accounting for recognised industry variables. This also helped clarify the domain in which the study was conducted (Eisenhardt, 1989; Peel, 2003). In this study, the Information Communication Technology (ICT) sector provided an ideal arena in which to study the innovation journey.

The industry was selected for this study as being a sector recognised as both innovation-aware and innovation-driven. However, the industry is also recognised as not always being able to realise effective results of this necessary innovation basis (New Zealand Trade and Enterprise, 2006). Innovation is regarded a critical requirement for operating in the ICT industry's rapidly changing environment. Competitive forces are felt more keenly than in some other industries with innovative activity at the forefront of management actions in ICT firms. Active pursuit of innovation and having to face the demands of constant innovation are paramount to continued existence.

\section{ICT in New Zealand}

The ICT sector is perceived by New Zealand Trade and Enterprise (NZTE) (2006) as an important contributor to New Zealand's economy and a key sector for economic growth prospects. Statistics by NZTE emphasises this. The industry consists of 8,100 businesses, NZ\$5.6 billion or 5.1 percent of GDP (Gross Domestic Profit), providing 44,000 equivalent full time jobs. Nine percent of firms have revenues of NZ $\$ 1$ million per annum.

Rapid developments in ICT and competitive activities drive the demand for constantly improved products and services whilst maintaining or increasing company profitability. Statistics New Zealand (2004) identifies the ICT industry as consisting of two of the top five industries for rate of innovation (Computer Services at $72 \%$, Telecommunication Services fifth at $56 \%$ ). Computer Services is second only to Electronic and Optimal Equipment Manufacturing (arguably part of the ICT industry).

The industry expectation - and norm - is of innovation agility by companies (New Zealand Trade and Enterprise, 2006), yet they are often unsuccessful in transforming innovations into useable outputs. The chasm between expectation and outcome is being addressed by the New Zealand Government though initiatives such 
as HiGrowth and the ICT Taskforce who are charged with, among other objectives, supporting entrepreneurial and innovation growth of the industry. Therefore, the industry provides a ready context for innovation activity and the realisation of innovation value within companies. In the strongly competitive ICT environment, successful companies are most often innovation-driven, therefore experienced in unlocking innovation value and so providing examples of innovation systems.

\section{Company Selection Rationale}

The companies were selected through information in the public domain (media releases, industry resources) as demonstrating successful innovation activities. The companies chosen for this study exhibit innovative practices, indicating that innovation systems of some form exist in the organisations. This is the paramount criterion in order to facilitate study of such systems. Hence, these organisations offered examples of the internal innovation journey that could be mapped, as desired for this research. The selection of three companies was an appropriate number which allowed for some variation (similarities and differences) within the scope of the study and within the constraints of time and of this level of academic study. The proposition being that three firms are likely to replicate or extend theory development (Eisenhardt, 1989).

A purposive sample was selected. Patton (1980) proposes that purposive selection provides "information-rich cases for study in depth" ( $p$ 169) resulting in information of importance to the research. The selection was purposive in that the companies were chosen on the basis of having developed innovation processes; were innovation-driven yet different enough to provide contrast and complement findings. Selection was based on replication logic (Tellis, 1997; Yin, 2003b). Yin (2004) advocates that three cases that replicate similar courses of events, result in reducing uniqueness which then, in turn, facilitates generalisation. Hence, three companies assisted to provide a more generalised argument; overcoming the shortfall of case studies and their outcomes being limited in application to other circumstances (Thomas, 2003). The use of sampling logic, drawing from a population, is considered by Tellis (1997) to be incorrect in case study strategy.

The three companies are all technology-based, yet provide dissimilar services and do not compete with each other. Therefore, the firms have substantially different suppliers and target different customers. Although the companies are typical examples of the overall sector, choosing organisations with diverse business 
operations challenges any overt commonality between them. This results in an innovation-to-outcome model being more rigorous while keeping the industry context valid.

Further, Yin (2004) advises that with multi-case studies, companies should be selected based on their normalcy. The three companies studied are representative examples of businesses in the high growth technology arena, being not overly exceptional or outstanding (Yin, 2004). The firms are innovative within the norms of the industry and are recognised for their innovative activities through winning innovation awards. The companies demonstrate relevance to the general industry rather than being unique or extraordinary, supporting replicability and generalisation.

In approaching firms, it proved important to frame the study in such a way that the senior person contacted appreciated the relevance and advantages to the company in participating. This approach is recommended by Van de Ven and Rogers (1988). Although this approach did not grant automatic access, necessitating a number of companies to be contacted, the companies who declined to be involved expressed interest in reading the study when available.

\section{The Companies}

The three companies who agreed to be case study subjects are all privately owned and are reliant on technology for their business offering. Overall their various products may be defined as generic. The organisations thereby differentiate and develop competitive advantages through their service provisions and business offering. These activities rely on innovation to grow, maintain and sustain market position. The three firms have serious competitors necessitating the vying for business and the securing of clients through innovative developments and actions.

The three companies are experiencing rapid growth and are perceived as successful members of the technology industry. The highly competitive market and propensity to innovation emulates Kanter's (1985) profile for enterprising, changeorientated organisations. This status and the problems currently being confronted by growth replicates Griener's (1998) first stage transition to second stage of organisational evolution. Griener stipulates in Five Phases of Growth (1998) that a company, in moving from the initial entrepreneurial stage to a more structured operation, requires the establishment of systems and order to be imposed on the somewhat chaotic working environment. In other words, a shift from the more 
disordered creative operating basis to a more stable foundation is necessary to sustain both day-to-day established business and the desire for continual growth. This aptly describes the three case study companies providing continuity of environment and focus, further supporting selection logic (Tellis, 1997; Yin, 2003a).

\section{One to One Interviews}

As the aim of the interviews was not to alter viewpoints or lead respondents but to reveal their thinking (Keats, 2000), the open manner of the interviews facilitated free discussion. Keats (2000) emphases the importance of interviews needing to be reliable, to be consistent and have validity, with questions and responses eliciting information that serves the purpose of the research. The developing of case study and interview protocol helped achieve these aims, as did the thorough checking with participants as to accuracy of interpretation. The latter helping to ensure a true account of phenomena is presented in analysis and report writing.

Before the interview the participants were provided with an information sheet outlining the study (Appendix A) and a Consent Form (Appendix B) which included gaining their permission to record the interviews. Three people per company were interviewed, three being the minimum to assist understanding the process from various perspectives. The most appropriate persons being interviewed in each case commenced with a senior executive with responsibility or involvement with innovation, for instance, the company's CEO or divisional general manager.

Known as elite interviewing (Marshall \& Rossman, 1999), starting with each company's most senior person gave access to the overall knowledge of the company's innovation activities. Specifically, due to their prestigious status, these senior executives would have strong familiarity and indepth involvement with the innovation systems. The initial interviewee (the senior manager) was then asked to recommend two other relevant people. Such identification of next interviewees being made using the snowball sampling technique (Bouma, 2000; Patton, 1980).

The recommended employees were considered suitable by the senior person as having extensive involvement with each company's innovation realisation process (Collis \& Hussey, 2003). Identifying these individuals would otherwise not have been possible without such direction. Similarly, gaining access to these people necessitated the senior executive's knowledge and permission to interview, which was gained at the time the recommendations were made. Interviewing three people 
per company reduced dependence on any one individual with the gathering of information from multiple sources helping to verifying authenticity (Tellis, 1997).

\section{Ethical Considerations}

Formal approval was granted by the Unitec Ethics Committee in August 2006. This was based on receiving letters from the three companies giving informed consent to participant as case studies. Participation by both companies and individuals was voluntary and confidential, with involvement being anonymous. The identities of the companies and individuals are therefore protected throughout this study.

\section{Interview Protocol}

Interviews commenced by addressing any ethical concerns and ensuring informed consent. Following, a series of open-ended, loosely structured questions (Carson, Gilmore, Perry, \& Gronhaug, 2001) were posed (see Appendix C for interviewer question guideline). This assisted in preventing the interviewer limiting or leading responses encouraging the interviewee to tell their story of the company's internal innovation systems. The format was standardised to provide a common guiding outline with the questions acting as prompts to the interviewer rather than specifically directed at the interviewee (Keats, 2000; Patton, 1980). Additional examples or questions were asked to gently probe for further information (Batonda \& Perry, 2001) and as checks to consistency of replies (Keats, 2000).

More of a conversation-style than formal questioning (Keats, 2000; Marshall \& Rossman, 1999), participants were asked to chart the company's process or processes. Further, to describe in their experience features and influences on their company's various systems and of the systems' effectiveness. Lists of barriers and supports were provided during the interview (drawn from the literature search, trialled and edited to assist comprehension) which were then reviewed for relevance to the company (see Appendix D). This list also provided a check against responses gathered earlier as some barriers reversed are enablers (re-worded) assisting to verify interviewees' consistency of responses.

Interviews were digitally recorded and transcribed supplementing notes taken during interviews and observations made while visiting the companies. Interview permission forms were not associated with interview results to support anonymity as well, each datum sheet (without names) was encoded to ensure confidentiality. 
Further, any distinguishing features are masked or not referred to in this study and no commercially sensitive information was gathered. Finally, findings were alluded to collectively (Peel, 2003) to limit recognition of individuals and companies. For purposes of confidentiality, reference to actual individual company results identifies the businesses only as Company $\mathrm{A}, \mathrm{B}$ and $\mathrm{C}$.

\section{Data Collection and Analysis}

Multiple case study strategy and protocol development were based on Yin's (2003b) design, consisting of the following stages:

1. Develop theory

2. Select cases and design data collection protocol

3. Conduct first case study; second case study; so on

4. Write individual case reports separately

5. Draw cross-case conclusions

6. Modify theory

7. Develop policy implications

8. Write cross-case report

Source: R Yin "Case Study Research", 2003, p. 50

Yin's (2003b) above process was applied in gathering data and complemented the requirement for evidence-sequencing in reliability and construct validity. Prior to interviews a pilot interview was conducted as a test, as recommended by Yin (2003b) which led to improvements in the interview guideline questionnaire and list of influences.

\section{Data Gathering}

Although a qualitative survey would also have provided rich data, the method limits the opportunity for participants to tell the story of a company's system. As indicated, this is better achieved through interviews within the company environment, which also supported the gathering of additional data. Data on the target companies were collected from two main sources: interviews and analysis of company information. The latter was distilled from companies' general information and innovation activity reports available in the public domain (the internet, including companies' web sites; media reports, articles). Additional background information was gained during site visits, conversations and interviews with relevant employees. Multiple-data sources enabled checking of validity (Eisenhardt, 1989; Tellis, 1997; Yin, 2003b) and a key feature of case studies (Hamel et al., 1993). 
Collecting such information provided a secondary level of data to support understanding of the various systems and to enrich interview findings (Miles \& Huberman, 1994). The range of data conforms to Yin's (2003b) list of six evidentiary sources. Triangulation was achieved through associating these sources of information with the primary data gathered from interviews. Although the study has some quantitative data, qualitative data dominates as to be expected in such a study (Kohlbacher, 2005).

The preference of this study, in appreciation of the ambiguity of the innovationto-outcome process and firms' unique ways of addressing the issue, was to be alert to whatever features became apparent during data collection. The objective was to be open to variances and nuances during research and to access each individual's perspective (Patton, 1980). The risk of pre-empting or limiting recognising companies' actual practices by using an existing, prescriptive model, for instance Cooper's (2001) Stage Gate ${ }^{\circledR}$ process was negated by this stance. Use of a rigid framework may have impeded deeper understanding, incurring the ignoring of some data, or constraining the collection of rich data (Collis \& Hussey, 2003).

This stance is also supported by Schroder, Van de Ven, Scudder and Polley (1986). In their study of innovation, Schroder et al (1986) were reluctant to commit to a particular process as potentially pre-supposing findings, preferring the inductive approach of non-attachment to a model. As such, in this study, it is posited that employing such a structured model may have distorted findings owing to their specific application. This reinforces the generic applicability of Rogers' (2003) Innovation-Decision Process model which provides broad steps and is able to be generalised. The model therefore facilitates the identifying of the actual, practical stages occurring in the innovation outcome journey.

\section{Data Analysis and Reporting Results}

Within-case investigation dictated detailed write-ups for each situation while reducing the volume of data (Collis \& Hussey, 2003) to be analysed later. The rolling analysis at times converging with data collection (Eisenhardt, 1989) with further information being sought while still on premises to support understanding. The field notes helped to better ground the study and provide insights that delayed analysis may have overlooked (Eisenhardt, 1989). 
Firstly, data reduction was undertaken to simplify and concentrate information (Miles \& Huberman, 1994; Rudestam \& Newton, 2001). This assisted in the organising and sorting (Miles \& Huberman, 1994) of findings. Data reduction developed into an iterative process during analysis revealing themes and patterns (Marshall \& Rossman, 1999; Patton, 1980). Potential influences on the system were tabulated quantitatively in binary yes/no responses. As well, participant's responses to individual points are quoted to assist comprehending the impact of these factors. Findings were consolidated, integrating data across cases to provide a narrative portrait. Inclusion of quotes from case study participants added qualitative insights providing support to data presentation and interpretation as recommended by Batonda and Perry (2001). The case study companies' systems were mapped showing the flow of events, providing visual representation of activities.

Analysis of results was structured around the theoretical framework of Rogers' (2003) model providing a priori reference (Collis \& Hussey, 2003). This model and other models identified during the literature review, together with research objectives, provided key themes forming the basis of the discussion chapter. Data display analysis (Miles \& Huberman, 1994) was used to render data visibly to recognise patterns, matrix tables and displays assisting to further analyse case studies (Miles \& Huberman, 1994). Cross-case patterns were identified then tested against other data to help limit false conclusions and pre-empt bias (Eisenhardt, 1989). Eisenhardt (1989) argues that cross-case comparisons compel investigators to reach beyond first impressions. Soy (2006) agrees that this improves probability of reliable and accurate findings through requiring investigators to consider different interpretations.

Results of each case are reported individually (Patton, 1980; Yin, 2003b) drawing on interview transcripts and associated data concentrating on the innovation-to-outcome systems operated by each company. Additionally, key events and features of the processes are presented (Patton, 1980). The next stage of analysis was a cross-case comparison. Data within cases were systematically compared and contrasted (Somekh \& Lewin, 2005) looking for similarities and differences to further reveal companies' systems and the variances thereof. Continuous development of understanding and interpretation occurred as data were analysed and truths revealed (Marshall \& Rossman, 1999). 
Triangulation occurred on two levels (Kohlbacher, 2005). Triangulation of data being beneficial to overcome bias, providing fuller detail (Collis \& Hussey, 2003; Yin, 2003b) and is considered characteristic of case studies (Tellis, 1997). Firstly, data gathered from multiple sources were analysed and compared to support validity and reliability. Double checking of interview transcripts for accuracy further enhanced reliability, with clarification being sought by participants if required. Secondly, application of pattern matching (Miles \& Huberman, 1994) was used to investigate the logic of patterns and consistency. These activities assisted in corroborating data analysis and research findings (Rudestam \& Newton, 2001).

Subsequently, theories were developed during data analysis aimed at answering objectives. Known as post factum theory or ex post facto (Wilson, 2005), this is the production of theory after gathering data. This replaces theory testing occurring when theory comes first (not applicable for this study) drawing on data analysis to develop findings. The iterative process of comparing data to theory resulted in theory (or theories) which best fits the data (Eisenhardt, 1989;

Kohlbacher, 2005). Further, cross-case findings relied on developing arguments or explanations supporting and linking with relevant literature and theory (Yin, 2004) to provide grounding for the study.

Yin's (2003b) four principles to assist best quality analysis were reviewed to further accuracy of findings, as cited by Umit (2005) :

1. That analysis relied on all relevant evidence.

2. All major rival interpretations were included.

3. The significant aspects of the study were addressed.

4. Prior knowledge of the subject was drawn upon to further analysis.

Finally, an emerging model of the innovation system was constructed from research findings. The innovation model was based on analysis of Rogers' (2003) model incorporating data from the three companies innovation system events and the features thereof.

\section{Conclusions}

In drawing conclusions, Bouma (2000) suggests there are two levels. The first level is to simply and clearly state what is disclosed by the data. The second, how this relates to the overall issues of the study. Hence, identification and consideration of various explanations for findings resulted in the most plausible explanations being 
offered (Marshall \& Rossman, 1999). These are detailed in Chapters Five (p. 105) and $\operatorname{Six}$ (p. 121).

\section{Limitations of Research}

As well as the limitations to case study strategy and interviews discussed earlier in this chapter, there are further short comings. The scope of this research was limited to three companies that have established innovation-to-outcome systems in the ICT industry. This industry was selected as providing indicators to innovatively successful companies as innovation agility is a pre-requisite for continuing growth in this highly competitive environment. The use of the ICT industry and its distinct nuances may limit generalisation to companies outside of the industry.

As discussed earlier, purposive sampling was necessary in order to research actual innovation outcome systems, as demonstrated by the chosen companies. Purposely selecting companies is problematic in that the organisations chosen may not be representative of the population (Trochim, 2006). The concern was addressed by randomly selecting a number of companies that were identified through media releases, innovation award notices and other relevant industry sources. A number of firms were approached with three agreeing to become case study subjects. This assisted in selecting companies that were representative of the industry within the specific criteria of displaying innovation realisation effectiveness.

Similarly, use of snowball sampling can further compound concerns with representiveness of the research. However, access to individuals who were directly involved in each company's innovation activities was necessary in order to gather worthwhile data (Collis \& Hussey, 2003). These individuals' identities are not in the public arena; hence knowledge of them required identification by the senior person at each organisation. (Trochim, 2006). As the names of these senior people were available in the public domain they were able to be approached directly to gain interest and permission to be case studies subjects.

Although multi-case studies are considered more robust than single cases, the selection of three is a minor sample potentially reducing general applicability. Similarly, the study purposely identified particular types of companies in a single industry as the objective was to identify and analyse internal innovation systems, which the sample companies demonstrated as providing. This differs from randomly 
selecting companies and industry to provide a wider context, yet may have lead to a lack of identification of effective innovation systems.

Additionally, qualitative data are subjective to researcher bias, as are interviews. In order to minimise bias a number of strategies were used including multiple sources of evidence to ensure validity, the use of models to support analysis and feedback from companies on preliminary results. Use of these tools aimed to reduce the risk of mis-interpreting findings or proposing misleading conclusions.

To summarise, a multi-case study approach was employed to answer the research question and objectives (outlined at the beginning of this chapter). Key employees directly involved in unlocking innovation value were interviewed to gather rich, qualitative data on the internal innovation systems. As part of each interview, a copy of barriers and supports drawn from the literature review was presented establishing how each company's system was affected accordingly. The results of responses to this list providing both quantitative and qualitative details on system influences. The interview data and other gathered information were analysed to identify patterns and themes, considering the various interpretations in conjunction with theoretical constructs. The following chapters present details. 


\section{Chapter 4 \\ RESEARCH RESULTS}

\section{Chapter Outline}

This chapter presents the results of the three company case studies drawing from the empirical research, comparison and analysis of data. The chapter commences with an overview of each of the case study companies, followed by interview findings and associated data from research conducted on each organisation. Subsequently, a summation of the results across the cases is provided. Interpretation and discussion of the results ensues in the next chapter including consideration of the results within Rogers' Innovation Diffusion Process Model (2003).

Results are presented in order of this study's objectives. The objectives being to identify innovation systems employed by the companies, event features, patterns and practices associated with successful innovation outcomes. As well, two objectives relate to ascertaining the presence of influences on system outcome success.

\section{Case Study Companies}

As previously presented, this study uses a multi-case strategy researching three companies in the ICT (Information Communication Technology) sector to collect research data. Interviews were conducted with people in senior management in order to understand the innovation systems each company uses and aspects associated with innovation outcome success. Note: to protect anonymity, only limited statistical and evidentiary information is provided on the companies.

Company $A$ is one of the top telecommunications companies in New Zealand, offering the full range of telecommunications services. It has been a leading actor in driving de-regulation of the industry. Established in 1996, the firm's turnover is in excess of $\$ 60$ million with offices throughout the country employing 170 people. The company is regularly listed on (frequently winning) innovation category awards. The organisation's ICT offering is a source of differentiation in the market and is primarily developed within the company, the firm rarely procuring externally produced telecommunications products. One of the main drivers of Company A's success has 
been its ability to create and provide cost-effective innovative solutions that others in the market are unable to easily emulate.

Company $\mathrm{B}$ is a technology based company offering data services and fulfilment. Clients include a major telecommunications company as well as other well known New Zealand businesses. The company is often recognised by the industry for its innovative activities, most recently having won two gold innovation awards for its service and software-driven provision. Employing over 60 people, the privately owned firm has a turnover in excess of $\$ 25 \mathrm{~m}$ and is in the top quartile of its industry sector. The organisation is recognised as providing leading edge innovative solutions.

Company $\mathrm{C}$ is a young subsidiary of a large New Zealand company with business ownership shared amongst the owner of the umbrella company and key staff. The business provides converged data and voice solutions, its range including internationally award winning quality products. The group of companies is recognised for its innovation activities receiving awards for its innovative offering. The company has over 75 employees with offices around the country and numbers some of New Zealand's well-known organisations among its clients. There is some export activity.

Together, the three companies represent innovative businesses that attribute success to realising value from innovations. Operating within the ICT industry, individually they pursue very different customer groups and provide services completely unrelated to each other. In other words, the companies' business offerings are dissimilar to each other.

Note: to clarify the steps and processes employed by each company, the actual systems were mapped in conjunction with interviewees. Participants later checked the system drawings and events each contained to ensure accuracy. 


\section{COMPANY A}

To understand Company A's internal innovation journey interviews were conducted with three people: the most senior employee of the organisation and two other senior executives, one heading the IT division, the other the marketing department. Firstly, to establish the firm's and interviewees' attitude to innovation, each person was asked to provide their opinion on innovation in business today. Company A's three interviewees agreed that innovation is absolutely critical to business and their firm specifically. They elaborated, stipulating that innovation is necessary to provide points of difference to other (competitive) firms, to ensure differentiation of their provision and market perception. Innovation is also considered important to maximise use of resources, "achieving more with less" (Interviewee A1).

\section{The Practical Innovation-to-Outcome Systems Employed}

Company A employs a variety of systems to unlock the value of an innovation. Selection of system depends upon resource demands including time commitment, whether within-division or cross-department and if the innovation is radical or incremental in nature. Additionally, the organisation has developed a specific system to encourage innovation in the IT department.

\section{Basic System}

Company A has a basic system (Figure 5), most often used for realising incremental innovation, innovations requiring minor or no resources. This type of innovation reflects the demand within the company for continuous improvement and the optimising of processes. Additionally, innovations directed through this system usually relate to single employees, teams or divisions. In other words, they have minimum impact on the business overall.

The basic system consists of a simple few steps aimed to rapidly lead to an outcome, which is to either implement the innovation or to proceed no further (terminate). The first version (System A 1, Figure 5), described by all three interviewees, explains the series of steps of what they consider is a casual, freeflowing process. The system commences with the idea generator speaking firstly to colleagues, then their line manager, or to the line manager directly. One interviewee referred to this as conducting a "sniff test" (interviewee A2) to test the concept's viability. If the idea is found favourable and is within the manager's delegated responsibility, it is immediately implemented. Interviewee A1 described the simple 
process as "Employees are constantly in touch with their local manager, there is no particular sign off unless it is required as outside of boss's delegated authority".

The company has two variations of the basic system. One department requires the idea generator to write the innovation on a sticky (Post $\mathrm{t}^{\odot}{ }^{\odot}$ ) note and attach it to an ideas tree. In this instance, portrayed by Interviewee A2, the concepts on the ideas tree are collected and reviewed weekly by team leaders who then decide the next stage. If the innovative idea has merit, it is implemented, if not, it is terminated. The innovations are department specific, examples cited were improvements to a manual process providing a beneficial time reduction, or the creation of a template for improved written communication. The company expects this type of innovation to be common and frequent occurrences throughout the organisation, quick to implement, constantly improving work activities.

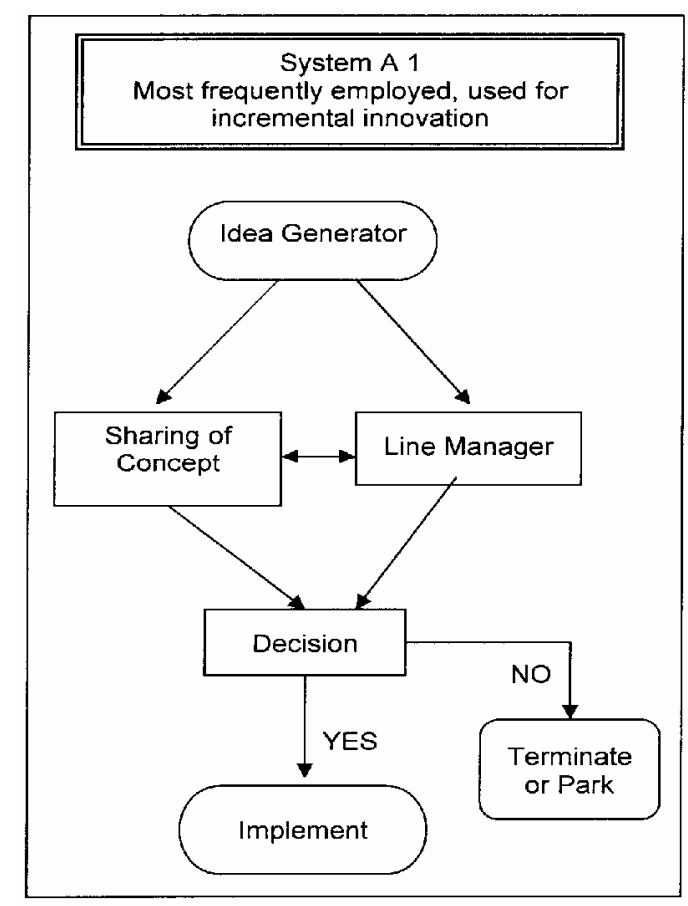

Figure 5: System A 1: Basic System

Another variation (Figure 6) builds on the basic system and is for innovative concepts submitted via the company's intranet. Interviewee A3 advised that in this process a manager regularly reviews these innovations, deciding each idea's worth. The innovative suggestions can have various levels of implications to the company; increasing complexity hence, the system includes additional steps as necessary. This version generally starts with testing the innovation on a trusted colleague or colleagues, or it is directly posted on the intranet. Gaining approval by the manager 
means the appropriate department, or departments, are advised of the suggestion. Again, if acceptable, it is immediately implemented. The department may revise the innovation to improve its suitability. If the innovation is more complex, and worthwhile, the product manager generates a business case for submitting to higher authority.

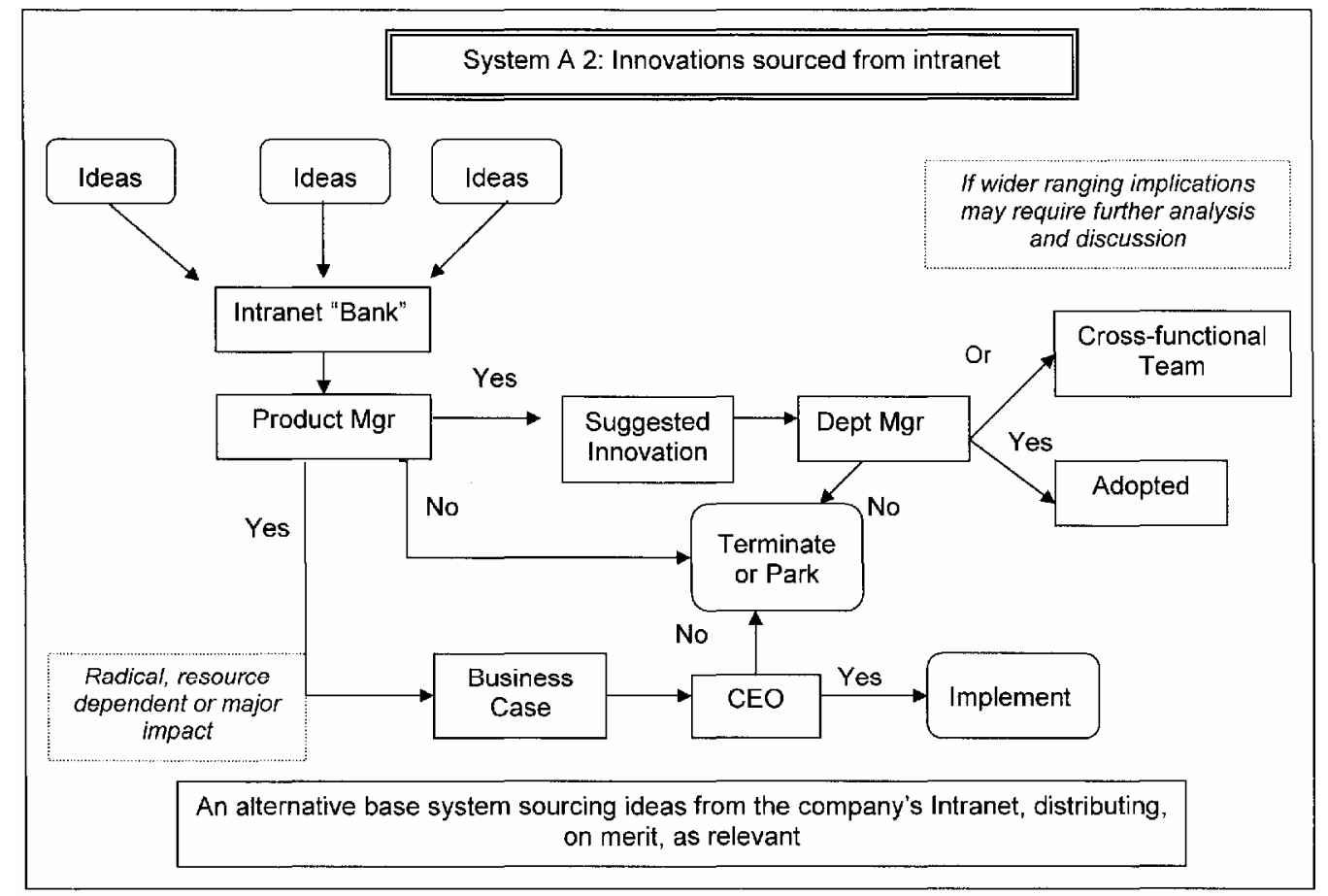

Figure 6: System A 2: Ideas sourced via company intranet

\section{System A 3: Some Resource or Cross-Department Demand (Figure 7)}

Interviewee A2 outlined the recently developed System A 3. Generally, this system is used for incremental innovations that have potential to amplify throughout the organisation, impacting on a larger number of people and departments and having greater implications. This system expands the basic system's (System A 1) stages including more people to minimise risk and maximise acceptance.

Additionally, radical innovations (if not resource-rich), it was explained, may also use this system. Interviewee A2 advised that System A 3 is an adaptation of Cooper's Stage Gate (2005b) system. The company is drawing on Cooper's model as a guide for this new process which aims to assist best resource allocation. Developing such a system has become necessary due to the company's growth and the resulting increasing demands on limited resources (especially monetary). 


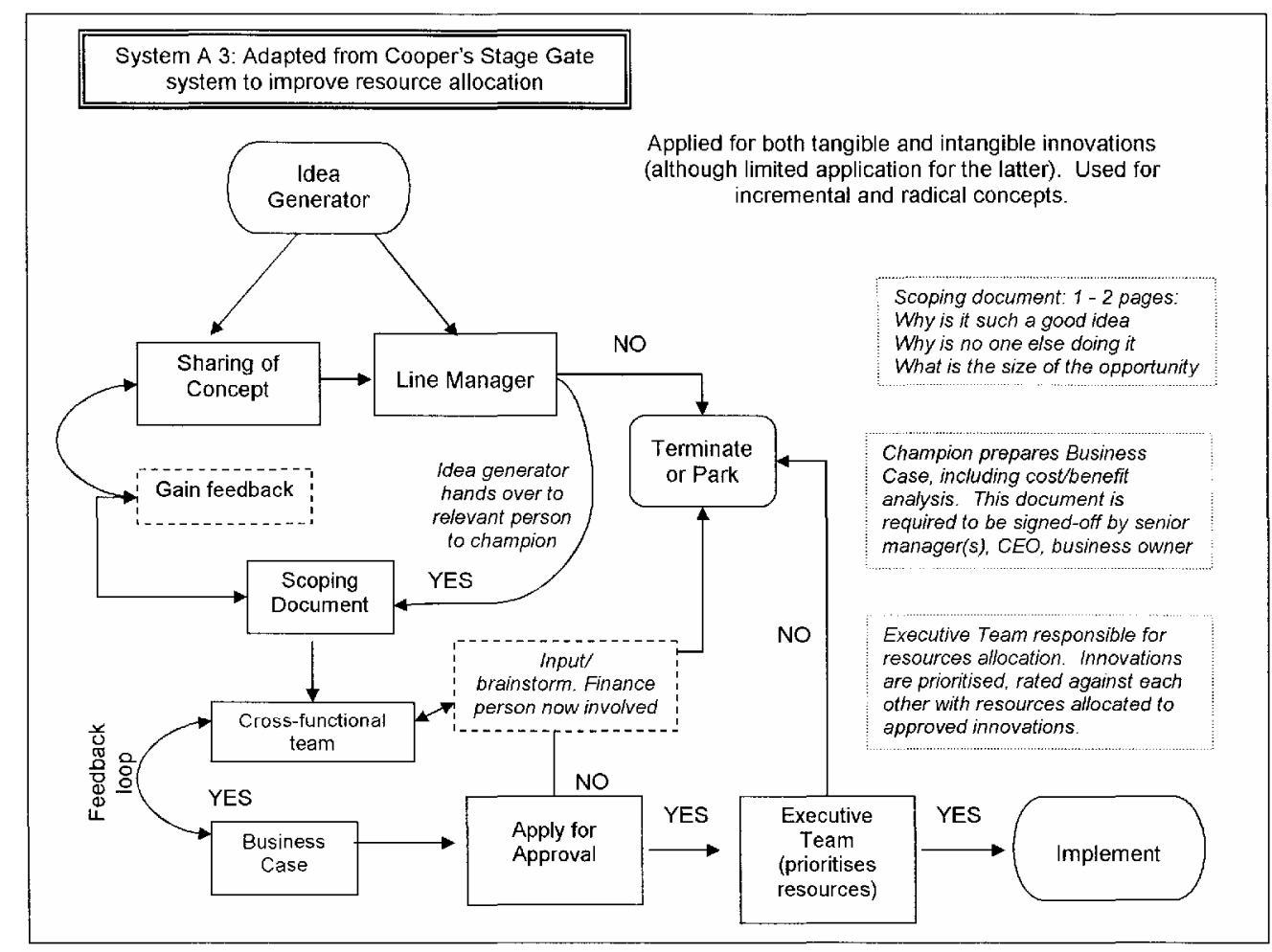

Figure 7: System A 3 System based on Cooper's (2005b) model

In System A 3, additional stages incorporate more meetings to gather a wider range of views and reviews. To paraphrase Interviewee $A 2$, the new system prevents prematurely committing resources, engaging necessary staff at each stage to ensure close inspection of risk. Involving all the people the innovation potentially impacts also assists in persuasion and implementation. As well, as can be seen in Figure 7, added stages include a depth of analysis not present in the basic system. Interviewee A2 advised that formalised documentation is necessary to facilitate acceptance and to better comprehend dangers. The system culminates in the executive team meeting to review the innovations on offer. These senior employees are responsible for allocation of scarce resources and decide on the fate of each innovation. 


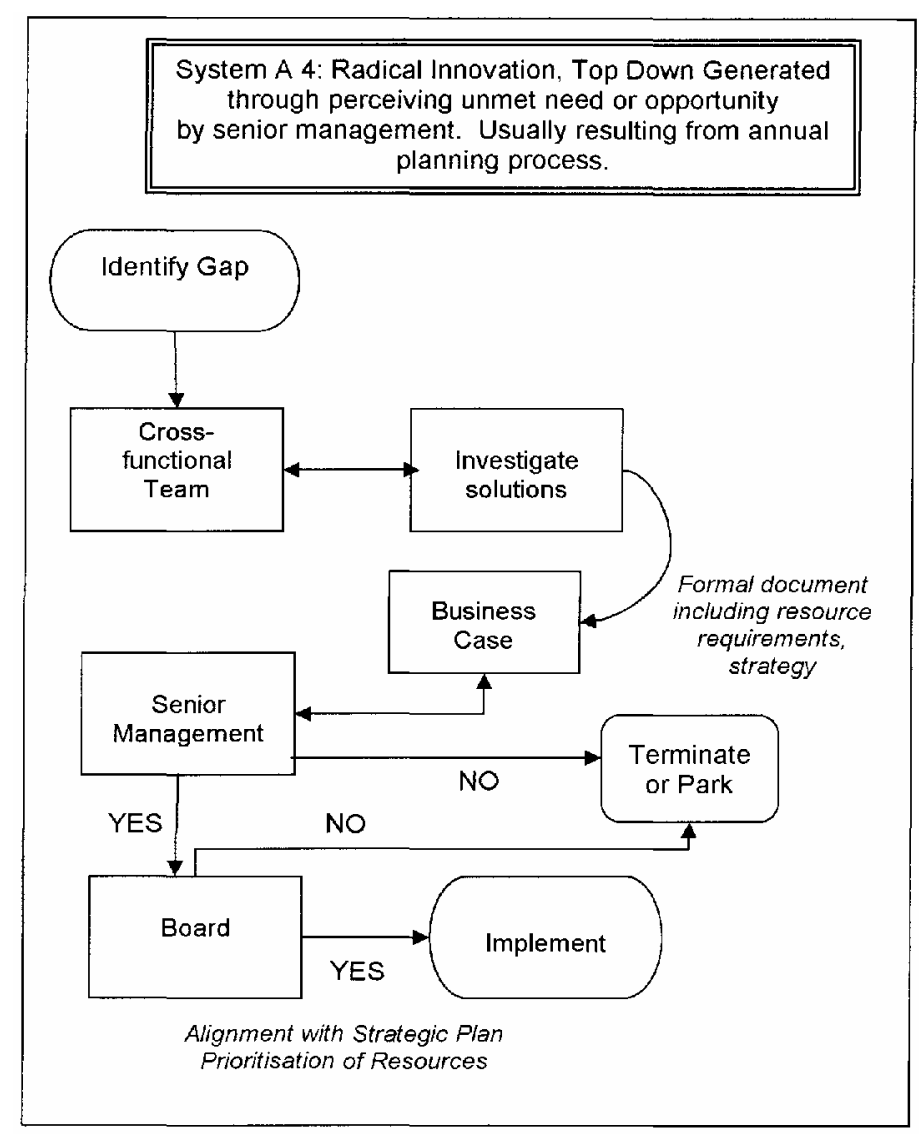

Figure 8: System A 4: Radical Innovation

System A 4: Complex. Radical, High Resource Demand, Serious Implications to Company (Figure 8)

Interviewee A1 advised that when an innovation has major implications to the organisation and is resource demanding, a separate system engages. This type of innovation, most likely radical in nature, is usually a result of purposeful intent by senior executives to meet a perceived gap in the business, industry or in customer demand. One interviewee explained that few staff have sufficient oversight of the company to generate significant insights, accordingly, generate far reaching innovations. These types of innovations instead rely on senior employees to create. On recognising a major opportunity or innovative imperative, the executives appoint a team to develop innovative ideas, investigate and analyse different options, selecting one innovation to then prepare a business case. The team then presents the business case to senior management. If successful, the final step is ultimately presenting the concept to the board for approval and allocation of often extensive resources. Overall, the system is similar to System A 2, with further stages to mitigate the possible extreme risk present in major application of resources. 
System A 5: Atypical. Encouraging Innovation Activity within IT Department

An annual event (Figure 9), known as Innovation Month, was described by two interviewees. The company developed this system to encourage the IT division which is seen as the innovation engine of the business. Twenty five percent of each IT employee's time for one month is dedicated to developing a working proof of an innovation after receiving approval from the head of department.

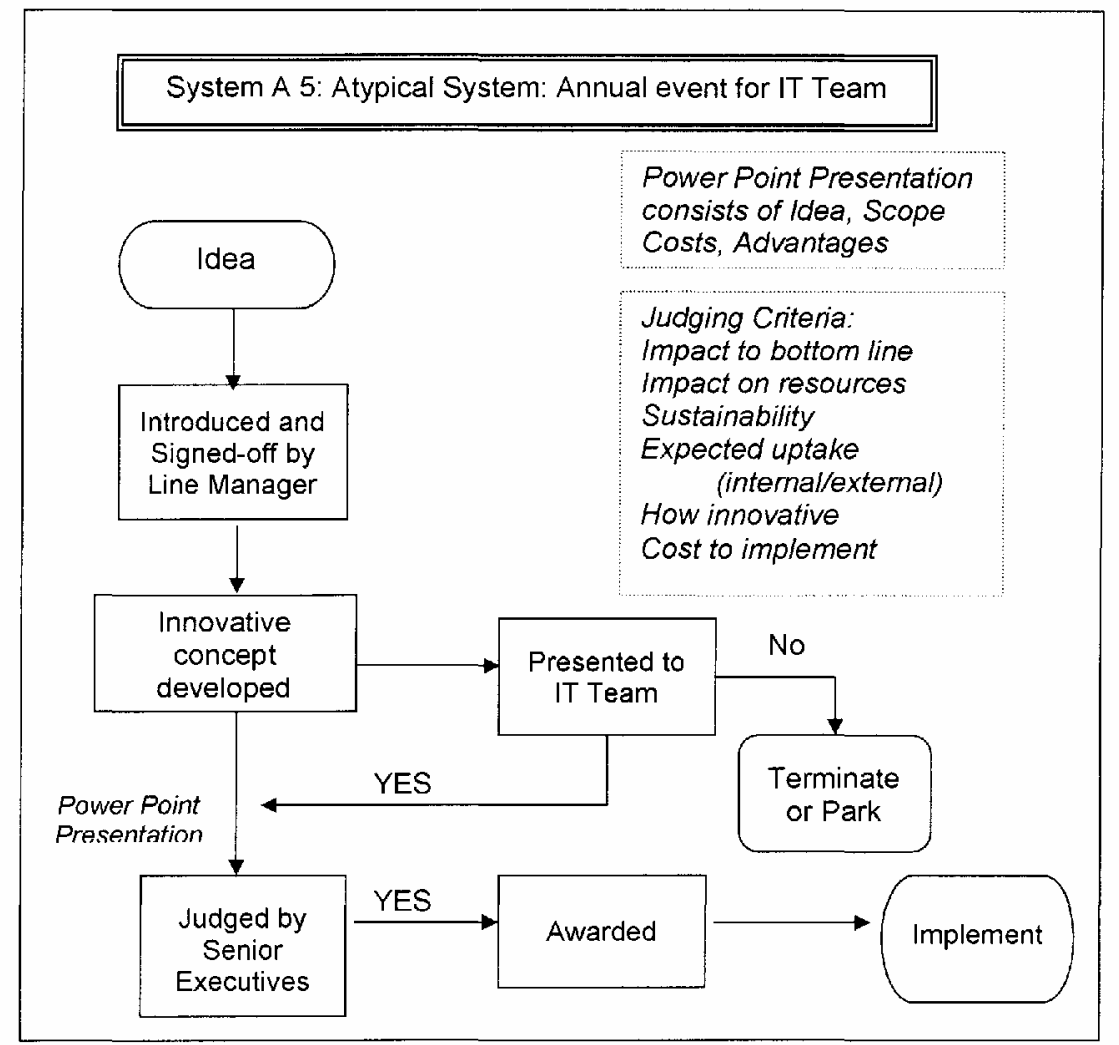

Figure 9: System A 5 (Atypical): Annual IT Innovation Event

Interviewee A3 advised that staff are provided guidelines to develop incremental "quick win" (Interviewee A3) innovations that require little resource. At the end of the month the IT department meets to present concepts to the rest of the team. The group selects the best three to then present to the executive team, the presentation consisting of a short (five minute) power point presentation, a minibusiness case covering benefits, sustainability and impact on the bottom line. The executives select a winner, rewarding accordingly; however, all three innovations may be implemented.

\section{System Versions and Stages}

Each of the three interviewees had different interpretations as to what comprised Company A's innovation systems. Whilst all three agreed to the simplistic 
System A 1, individual interviewees offered versions of the basic process (differing sources of innovation ideas), System A 2 and System A 3 and the radical system (System A4) furnished by the most senior of the three people. Two interviewees provided System A 5. (Figures 5, 6, 7 and 8 respectively).

Interviewees' described the stages in the systems sequentially. They elaborated, stating that often independent stages run in rapid succession. In other words, one step blending into the next if urgency demand rapid realisation or the innovation in its simplicity enabled this to happen, for example an obvious system improvement.

\section{Features of Company A's Systems}

A feature of Company A's systems is non-proceeding innovations. At all times, regardless of system, if the innovative idea is rejected it is terminated or parked. Interviewees' refer to parking an innovation as, regardless of merit, it is outside current activities, too resource demanding or inappropriately timed. If generated by or related to the IT department, the concept may be stored on the company's intranet.

The innovations stored on the intranet can be accessed, sometimes together with the reasons for discontinuing. Likewise, innovations that have been implemented that have proved disadvantageous may also be stored this way. Other departments in the company do not use this facility for capturing rejected concepts. If the innovation is verbal, no further record is held. Therefore, if an innovation becomes relevant or opportune, reliance is on the idea generator to revive it.

There are various identifiable determinants for system choice, such as timing of innovation, type of innovation and innovation form. Timeliness or urgency can influence system selection. For instance, continuous improvement innovation that is quick to install and has little influence outside of individual or department application utilises System A 1 (Figure 5). However, if a unique opportunity presents itself that has wider influence, or may impact the entire company offering major revenue potential, it too will be processed through System A 1. In this latter situation referred to by Interviewee A2 as "All hands on deck, the whole company going for it" - the steps otherwise employed to minimise risk are omitted in recognition of urgency of innovation outcome. There is enrolment of all necessary personnel and the 
instigating of short time frames to inspire a quick response to realise the value present in such a major innovative action.

\section{Type of Innovation}

Company A expects staff to provide suggestions for continuous improvement or incremental innovations which result in better operating efficiencies, systems and processes and market offering. These types of innovations may impact on revenue growth; however, the tendency is for cost savings rather than income generation. Owing to the lack of resource demands by such innovations and the small area of influence, continuous improvement innovations are actualised as quickly and simply as possible using the basic systems. Radical innovations, however, are processed by System A 3 or A 4. Generally, radical implies change which in turn implies resource requirements with a greater degree of cost/benefit analysis essential. Additionally, radical innovations may require consent of the board if there are any requirements for substantial financial investment.

\section{Tangible and Intangible Innovations}

There are no separate systems for tangible and intangible innovations. However, one interviewee admitted that experience with application of Cooper's system suggests the system has limitations in regards to realising intangible innovations and that the lack of suitability to intangible innovations was a component of System A 3 that required refinement. A comment also was that tangible innovations are given greater importance owing to their more measurable impact on revenue. Intangible innovation improvements were proposed as sometimes providing greater overall benefit but being not so evident in resulting (immediate) effect on the bottom line. Yet, if the latter is relatively innocuous, does not require resource investment, or saves having to employ extra staff, it may be implemented without further ado.

\section{Company A's Practices Supporting Innovation Process Management}

Interviewees described four critical areas impacting on Company A's successful innovation outcome: idea generation, culture, access to decision makers and growth.

\section{Idea Generation}

A corollary of culture, idea generation is encouraged in an environment that supports and celebrates innovation. Innovations may happen as part of reviews of 
ongoing activities or may result from planned efforts to stimulate the business or address gaps, driven by growth or to maximise existing resources. The senior person interviewed advised that radical innovations rarely happen on their own accord, that there is conscious effort made to identify opportunities, consider what threats need to be confronted, and internally, areas that may, in an executive's view, not be operating optimally.

Ideas are also intentionally requested from staff. An annual planning cycle involving all of the company solicits innovative ideas, incorporating them into planning documents. This is in addition to the ideas tree, the intranet email repository and direct communication between individuals seeking to improve company activities. Other sources of ideas include reviewing international and local industry activity: What is been done well, what is not being done at all, and why. Interruptions of management time are welcome from staff wishing to share an idea and are perceived as indicators of people thinking innovatively. Management view these interruptions as manifestations of an innovative culture supportive of achieving of company objectives.

\section{Culture}

The interviewees all advised that maintaining a flat organisational structure and minimising hierarchy are critical to support innovation and that the company appreciates the importance of both. As well, limiting bureaucracy to maintain free movement of ideas and communication is well recognised. The office layout is generally open plan with some separate offices - operating on open door policy - for senior executives. Interviewee A1 and A2 advised that they believed the company is still small and integrated enough to facilitate direct approach by staff to senior management. The relevance of such a culture in supporting innovation is exemplified by Interviewee A1's comment:

It is designed to keep reinforcing that one of our core values is innovation and if you want to be recognised, you need to be constantly thinking not just about your core day-to-day job but about how on earth we can sweat the assets, how can we get more out of what we've got.

Growth is hampering the company's previous entrepreneurial culture which the participants referred to as inherently flexible, nimble, responsive to opportunities and willing to take risks. Company $A$ is cognisant of the difficulty of retaining an 
innovative culture under increasing pressure of daily business. In the rapidly growing company concerted management efforts are now required to artificially stimulate ongoing generation of innovation concepts. The expectation is of management leading by example so that staff know innovation is important, with ideas given consideration and credit going to idea generators. As described by Interviewee A1:

People see that those who are innovating get recognised, those that aren't don't prosper to some extent. That the behaviours are modelled, in terms of willingness of senior management as willing to jump in what are clearly innovative ideas. There isn't a policy that says we are not willing to take risks.

This characteristic of the culture fosters innovation pro-activity and counters any reluctance to express ideas; the objective is to convey that no fault or blame will be attributed if an innovation is unsuccessful. The environment is conducive to new ideas and resistant to incumbent activities being untouchable, openly acknowledging that improvements are always possible.

\section{Decision Makers}

In all of Company A's systems the involvement of decision makers during initial stages facilitates innovation outcomes. Involving decision makers early eliminates the unwelcome surprise element that may well lead to negative reactions. In some situations, for instance resource demanding innovations, direct discussion of the innovative idea with a senior staff member abbreviates the process, with a supportive senior person over-riding possible impediments.

\section{Growth}

Interviewees all expressed awareness that growth is changing the organisation, diluting Company A's entrepreneurial spirit. The more established company does not desire to lose its innovative bias and the resulting competitive edge and growth. Previously, the young start-up company could consider risky innovations without heed to consequences to established clients, as the young company didn't have many. Furthermore, the company is conscious of the need to more carefully vet ideas to minimise poor investment of pressured resources or the compromising of current activities. A more operations-focussed leader has been employed to run and maintain the business, protecting the core revenue stream 
provided by retained clients. This new appointee complements the entrepreneurial owner who continues to drive the innovative aspect of the company.

It seemed to be manageable when we were small when you don't have the core of customers and day-to-day work that happens when you get to a certain size. You know your existing customers take more and more time to look after so your ability to innovate and disrupt the market and do all those things are diminished because you are starting to get a bigger stake in the status quo. (Interviewee A1).

The above quote exemplifies Company A's act of balancing incumbent business with continuing growth, stability with change (as a corollary of innovation). The quote also refers to the importance of not being consumed by day-to-day work and maintenance of core business at the cost of innovation. Interviewees remarked that it is imperative to generate and progress innovations that provide differentiation and service improvements. As the company grows, so does the number of projects, increasing conflict between innovating and managing daily business.

Additionally, sustaining the business and continuing growth has meant a shift from the flexible entrepreneurial approach operating on short time frames to planned strategic development. With increasing staff numbers, the need to convey the company's longer term objectives becomes essential to prevent the appearance of confused random activities and incoherence of direction. The company has formulated a clear strategy intending to offer a certain amount of flexibility to take advantage of innovations and opportunities, whilst maintaining a course of action so that employees are all proceeding in the same direction, operating within the same context.

Yet growth also means optimising processes. Opinions were voiced that less effective systems may be possible when small. However, the bigger the company the more efficient the systems need to be to manage greater operational complexity. Awareness of the factors of growth, for instance the demand for greater efficiencies, stimulates innovation in this area. Similarly, interviewees advised that the larger organisation, the slower the innovation process. However, they felt that the full extent of this is yet to impact explaining that a larger organisation provides more resources, greater variety of experiences and opinions to support successful innovation activities. At the same time resources are more thinly spread throughout 
the company, with more people involved with innovative developments which can result in delays. As well, there is a greater need for cross-functional co-operation, potentially preventing successful innovation outcomes.

A frequently used word by interviewees was priorities, the need to identify the best use of resources, pressure on limited resources an increasingly major issue as Company A has grown. Interviewee A2 explained that the first priority in judging an innovation's importance is its return on investment. Decisions were made on the value of the innovation to increase revenue, generate income supporting growth, leading to re-investment in further innovations.

One of the outcomes of the small entrepreneurial company transitioning to a more established business has been the requirement to provide a more structured innovation-to-outcome system. The current one was now proving less effective, as Interviewee A2 described it, "we are horribly enough on the structured path". Cooper's Stage Gate (2005b) model provides a structure that is being adapted and modified to help Company A prevent resource wastage. In particular, Cooper's model is seen as having the necessary interventions to facilitate clear decision making.

Despite rapid growth, the company continues to retain agility in decisionmaking, as apparent by the following quote (Interview A1):

At the end of the day, this is a proprietarily driven company, a small board, small set of owners and if we want to do something, we just do it. Quite flat with little clumps. Most of the big decisions are made so you don't have to convince lots of people and build consensus in that sense. But it means with that small group, if the whole tier doesn't support you, you have to wonder if you misunderstood something. But at the end of day, that small group can decide to do it anyway.

This sentiment was expressed by the senior executive conveying the company's continuing innovation-activity imperative despite growth demanding formalisation. The two other interviewees made similar comments reinforcing the internal view that the size of the company is still not as great as to prove overly cumbersome to innovation realisation. 


\section{Innovation Journey Influences}

Interviewees were presented with a list of barriers and supports (p. 28 - 31) to the realisation of innovation in an organisation, identified during the literature review. They were asked to advise whether they had experience of these in the company with opinions varying on their presence. The responses are depicted in a Table 4 supplemented with interviewees' comments, as follows.

Company A: Barriers to Innovation Value Realisation.

Indicated by respondents' full agreement, competing priorities due to finite resources and resource constraints was considered the biggest obstacle, with the company facing an endless list of opportunities to consider. Further unanimous agreement was expressed on:

- That there was no issue with organisational resistance to change or cultural inertia. In fact the opposite, although one interviewee had started to experience some lack of support recently which he attributed to the number of people now employed, together with daily pressures.

- $\quad$ All agreed that the company did not reward innovation (other than the specific exercise designed for the IT division), with one person remarking that it is difficult to ascertain whether innovative ideas were not being proposed owing to the lack of rewards or recognition.

- $\quad$ The difficulty of not being able to complete projects or find time to consider improvements due to the pressure of current duties.

Interviewees consider some barriers as irrelevant to Company A. These particular barriers do not prevent realisation of innovation in the organisation (evidenced by the sequence of negative responses to their existence: NTable 4, p. 68). For example, misallocation of finances.

On the issue of encouraging status quo and protecting existing practises, there was a division of opinion. The senior person was not aware of this occurring, whilst the others had had some experience of it. Similarly, the two less senior staff had increasingly experienced internal politics as the company has grown; however, it was expressed that a strong business case can overcome most resistance. 
- One interviewee remarked that there are more processes that do not support change (owing to growth), sheer size reducing cross-division functionality, with work volume compromising receptivity to innovation.

Table 4: Company A Interviewees' responses to barriers in the firm

\begin{tabular}{|l|c|c|c|}
\hline & \multicolumn{3}{|c|}{ Interviewees } \\
\hline Barrier (in no particular order) & A1 & A2 & A3 \\
\hline Competing management priorities. & Y & Y & Y \\
\hline Mis-allocation of finances. & N & N & N \\
\hline Rewards and incentives. & N & N & N \\
\hline Encouraging status quo. & N & Y & Y \\
\hline Management tying up critical resources better allocated. & N & Y & Y \\
\hline Bandwagon effect. & N & N & N \\
\hline Fear innovation cannibalising existing products. & N & N & N \\
\hline Management inability to implement innovation. & N & N & N \\
\hline Processes that do not support change. & N & Y & N \\
\hline Innovation introducing contradictions. & Y & Y & N \\
\hline Decision making by consensus. & N & Y & N \\
\hline Multiplicity of meetings to decide. & N & Y & N \\
\hline Preoccupation with current activities and markets. & Y & Y & Y \\
\hline Short term focus and/or an excessively internal focus. & N & N & N \\
\hline (Inappropriate) Strategic orientation. & N & N & N \\
\hline Internal politics. & N & Y & Y \\
\hline Organisational resistance to change and/or cultural inertia. & Y \\
\hline Lack of support to adopt change. & N & N & N \\
\hline
\end{tabular}

Key: $Y=Y e s$, agree with statement, barrier is experienced in organisation $\mathrm{N}=\mathrm{No}$, not experienced in organisation

- That innovation introduces contradictions promoted two to agree that it was increasingly difficult with company growth, although another had not experienced it. However, decision-making by consensus was generally felt not to be an issue as full support is not necessary to further an innovation.

- Multiplicity of meetings for decision making provoked divided opinions, from outright 'no' through to one person remarking it had become a 
scenario to "die a death by a 1000 meetings" (Interviewee A2). One interviewee's experience had taught him that uncertainty about an innovation's merit results in a greater number of people becoming involved. It was felt that when a meeting about an innovation has $8-10$ people then generally the idea is not going to succeed, usually because of a lack of a champion to drive the process.

\section{Company A: Enablers to the Internal Innovation to Outcome Journey.}

Conditions for transforming innovation into outcome were readily recognised as present in Company A. There was full agreement on the majority of enablers actively in place in the firm (sequence of $Y$ responses by all three people, Table 5). Specifically, although enablers were listed randomly, the first one (congruent with the list of barriers): Having sufficient resources was perceived as the key factor to realising the value in innovations. Another key requirement to successful innovative enterprise is, confirmed without hesitation by interviewees, the importance of a champion to support innovation. This could be the idea generator, generally though it is more often one of the senior management team due to the skill base required, knowledge and influence in the organisation. This also relates to the influence a senior person has in accessing resources. As well:

- $\quad$ Consistently, there was immediate acknowledgement that Company A's culture is supportive of innovation vision. This is seen as an important impetus, one person observing that it is a major contributor to the organisation's success. Similarly, the enabler: Aligning innovations with previous successful ones, is often utilised to overcome resistance, that this is perceived as a standard function of innovation implementation.

- Respondents generally believed that training staff to encourage innovativeness or responsiveness to innovation was an oxymoron. Sentiment was expressed that people cannot be trained to be innovative, rather it is dependent on the company's environment to stimulate, facilitate and support creative thinking that in turn generates innovation. Interviewees expressed experience with brainstorming as an effective method to employ to encourage innovation. 
Table 5: Company A Interviewees' responses to facilitators in the firm

\begin{tabular}{|l|c|c|c|}
\hline & \multicolumn{3}{|c|}{ Interviewees } \\
\hline Enabler (in no particular order) & A1 & A2 & A3 \\
\hline Having sufficient resources to support innovations. & $\mathrm{Y}$ & $\mathrm{Y}$ & $\mathrm{Y}$ \\
\hline Access to funds innovative ideas not requiring approval. & $\mathrm{Y}$ & $\mathrm{Y}$ & $\mathrm{Y}$ \\
\hline Rewarding successful innovation. & $\mathrm{Y}$ & $\mathrm{Y}$ & $\mathrm{Y}$ \\
\hline Overarching team to drive process. & $\mathrm{Y}$ & $\mathrm{Y}$ & $\mathrm{Y}$ \\
\hline Having a champion management. & $\mathrm{Y}$ & $\mathrm{Y}$ & $\mathrm{Y}$ \\
\hline Training staff to encourage innovativeness. & $\mathrm{N}$ & $\mathrm{Y}$ & $\mathrm{Y}$ \\
\hline Drawing on customers' experience. & $\mathrm{Y}$ & $\mathrm{Y}$ & $\mathrm{Y}$ \\
\hline Customer interrelationship by "backroom" personnel. & $\mathrm{Y}$ & $\mathrm{N}$ & $\mathrm{Y}$ \\
\hline Free flow of information. & $\mathrm{Y}$ & $\mathrm{Y}$ & $\mathrm{Y}$ \\
\hline Protecting innovation from bureaucratic limitations. & $\mathrm{Y}$ & $\mathrm{Y}$ & $\mathrm{Y}$ \\
\hline Setting goals for innovative achievement. & $\mathrm{Y}$ & $\mathrm{Y}$ & $\mathrm{Y}$ \\
\hline Encouraging a long-term perspective. & $\mathrm{Y}$ & $\mathrm{Y}$ & $\mathrm{Y}$ \\
\hline Culture supportive of innovation vision. & $\mathrm{Y}$ & $\mathrm{Y}$ & $\mathrm{Y}$ \\
\hline Cultural pride in company's (innovative) achievements. & $\mathrm{Y}$ & $\mathrm{Y}$ & $\mathrm{Y}$ \\
\hline Accepting failure. & $\mathrm{Y}$ & $\mathrm{Y}$ & $\mathrm{Y}$ \\
\hline Organisational structure and cross functionality of divisions. & $\mathrm{Y}$ & $\mathrm{Y}$ & $\mathrm{Y}$ \\
\hline Individual perspective of innovation. & $\mathrm{Y}$ & $\mathrm{Y}$ & $\mathrm{Y}$ \\
\hline Innovation introduced as complementary to previous. & $\mathrm{Y}$ & $\mathrm{Y}$ & $\mathrm{Y}$ \\
\hline Participative or team style of management. & $\mathrm{Y}$ & $\mathrm{Y}$ \\
\hline
\end{tabular}

Key: $Y=Y e s$, agree with statement, enabler present in organisation $\mathrm{N}=\mathrm{No}$, not experienced in organisation

With most facilitators to innovation realisation present in Company $\mathrm{A}$, together with the lack of barriers reflects the innovation bias and success of their activities. The interviewees all expressed concern that growth may hamper the success of innovation endeavours and actively mitigate to reduce barriers in continuing to realise innovation value. 


\section{Innovation and System Reviews}

Although specific reviews of innovations themselves are rarely undertaken, all systems operating in the organisation experience reviews on an ongoing, ad hoc basis as part of the drive for continual improvement. These exercises essentially include implemented innovations, ascertaining value and implementation success. The recent adaptation of Cooper's (2005b) Stage Gate system is indicative of these exercises. The company continues to pursue efficiency and revise applicability of systems to optimise innovation outcome.

\section{Success of Current Systems}

One interviewee expressed great fondness for some facets of the previous, flexible systems. However, he also recognised that it was no longer suitable to the larger organisation owing to volume of employees, competing resources and operational constraints. Standardisation and structuring is seen by interviewees as being more efficient and essential to the rapidly growing larger firm. The necessary transition summarised by Interviewee A3 as innovation realisation being:

$A$ very different process to what we go through compared to when we were a lot smaller. When smaller, one person can drive almost any innovation through to delivery, but now we have certainly added a lot more processes to be able to achieve the same results with more people having to work together.

Altogether, the presence of various innovation systems in the organisation highlights the company's focus on being innovatively agile and innovation focussed. The firm is structured to support these activities with management aware of the requirement to continually motivate innovation, innovation being respected as a cornerstone to the company's success. 


\section{COMPANY B}

If you aren't innovating and growing as an organisation, the people, and you as a leader, get bored and complacent. People and the good things that have taken you to where you are, leave, the essence is lost. Organisations need to innovate, if your market is changing then you need to be innovating, driving from within. Growing - financially - a bit more fundamental to support the team. They are only there because it is good for them. Challenges, fresh things to think about are important, and that comes from innovation. Becomes a circle. You grow, they grow, thinking about challenges, having ideas and ideas aggregate, then new ideas, new growth. (Interviewee B1)

The managing director provided the above quote which summarises the importance of innovation to Company $B$ and how innovation is the basis of the culture. The company's interview participants perceive innovation as a multitude of things including innovative thinking, a characteristic of the company that clients find attractive and an indicator of the organisation's ability to provide novel solutions. Fundamental to the business, innovation is seen as a key component of the company's success to date. Two interviewees commented that the external view of the firm was that it was solid and reliable yet providing innovative solutions that set it apart from competitors.

\section{The Practical Innovation-to-Outcome Systems Employed}

Interviews were conducted with three individuals, the managing director, general manager and senior account manager. Altogether, they described four versions of innovation systems that support the company's definitive creative bias and determination to pursue continual improvements. The systems and stages are not rigid. Interviewees see this as encouraging the free-flow of innovation, minimising barriers to encourage frequency of ideas and ease of sharing innovative concepts. The first of the four systems, described by all three interviewees, is the most basic and the most frequently engaged (Figure 10). Interviewee A3 described it as "A very loose process, one of the reasons we do so well is that we are not tied into having to go through a rigid process to get anything done". 


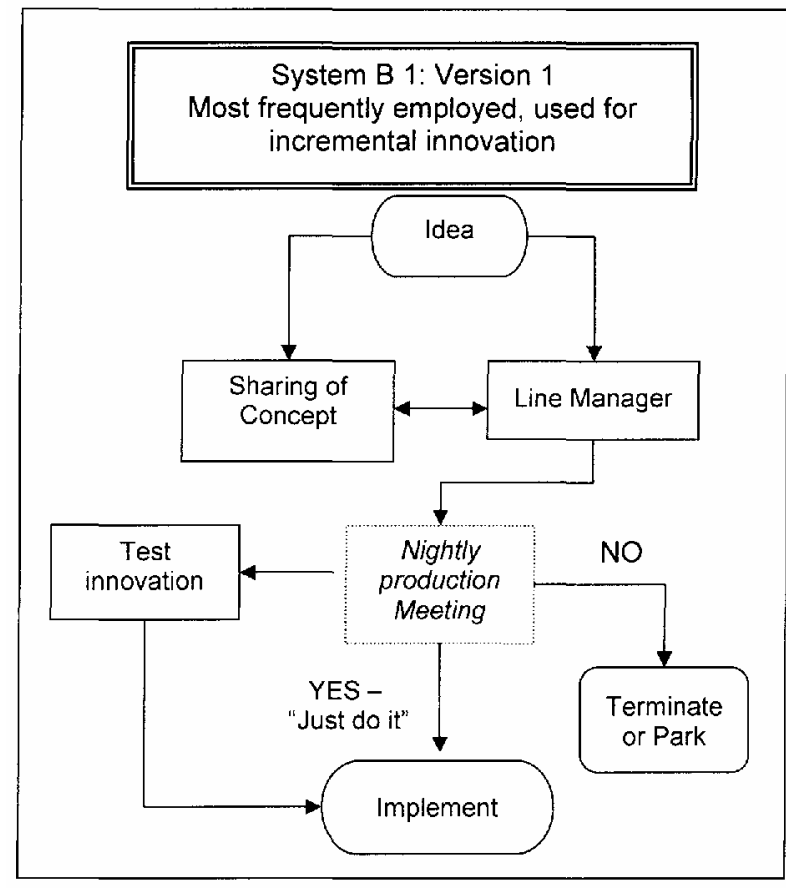

Figure 10: System B 1, Version 1

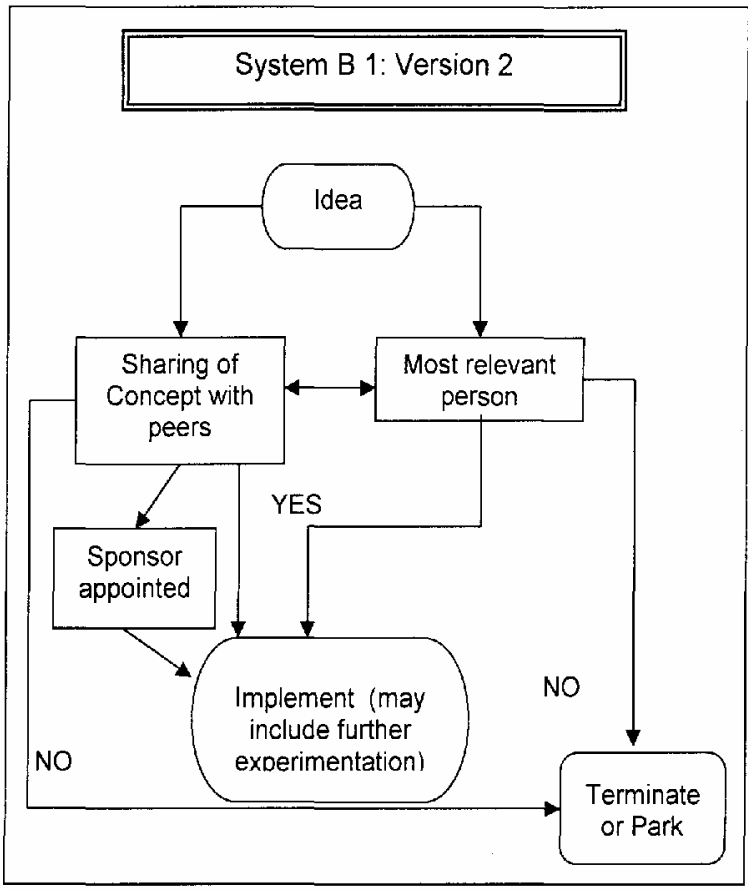

Figure 11: System B 1, Version 2

The simple series of events commences with the idea generator working through the concept, undertaking a casual or "intuitive" (Interviewee B3) analysis (benefits, costs), then speaking with fellow employees (peers) or directly with a senior relevant person to air the concept. The relevant person is either the manager the idea generator reports to, or the manager of the area most likely to be impacted by the innovation. The line manager then engages the next step by either encouraging implementation or requesting further investigation. If the idea is not suitable, it is terminated. The majority of incremental innovations generated in Company B do not have resource requirements; consequently, such innovations are immediately implemented. The decision to implement is based on apparent benefit and negligibility of negative consequences.

The system may include introduction of the idea at the nightly work-in-progress meeting. If the concept is time-limited, the innovation and analysis of costs/benefits are directly discussed with relevant personnel rather than wait for the evening meeting. In either case, the meeting decision is either to implement or terminate.

Interviewee B3 described a derivative of the basic system (Figure 11) which is similar in most ways to the System B 1 (Figure 10) with the addition of a sponsor. In this instance, the process starts the same with the idea being shared with peers or 
directly discussed with the relevant manager. The decision to proceed or not is usually immediate. The difference between the two basic systems then occurs. Appointing a sponsor (champion) may be necessary to oversee implementing the innovation if its complexity requires detailed instruction or there is involvement of several divisions necessitating oversight by one individual.

\section{System B 2: Including an Expert}

If the innovation is more demanding on resources or requires greater consideration, the basic system (System B 1) has an added element, the inclusion of an expert to maximise potential benefit (Figure 12). Involving an appropriate expert (described by interviewees as someone within the company who has expertise in facets relevant to the innovation or provides expertise in implementing) can be necessary to give advice on the viability of the concept. Additional to System B 1 Version 1 is that in $\mathrm{B} 2$ a more intense cyclic process of benefit and cost analysis, testing and refining the innovation before fully implementing.

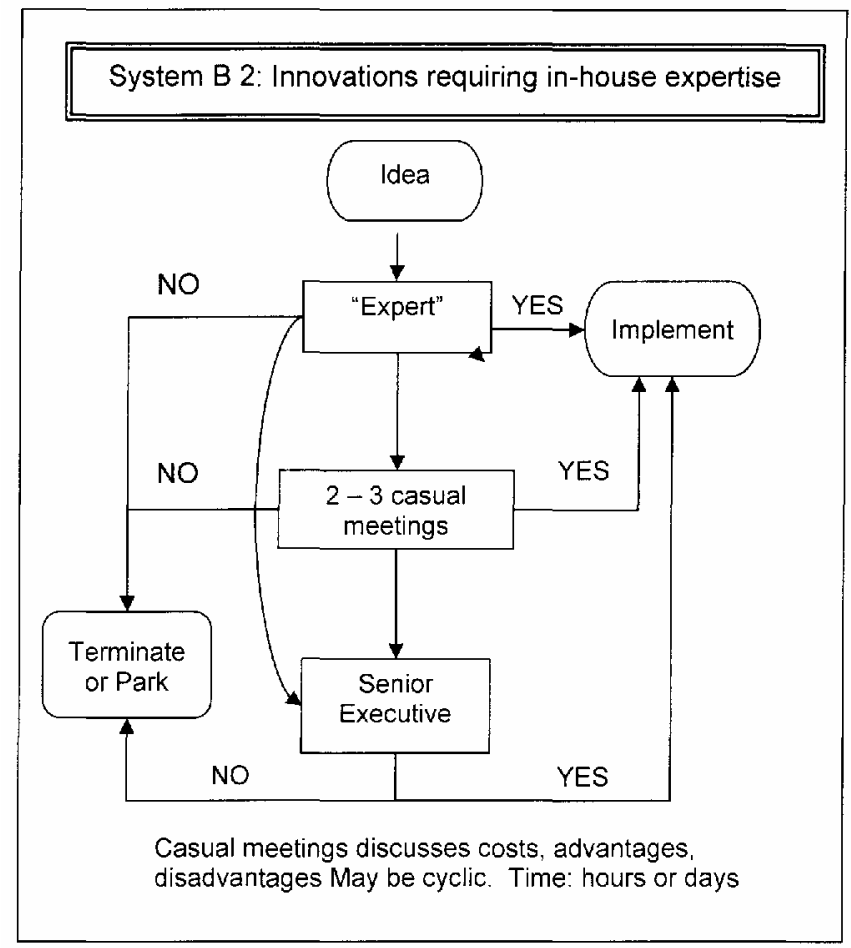

Figure 12: System B 2, Inclusion of an expert

\section{System B 3 Radical Innovation}

A fourth system is employed for resource demanding innovations which impact across the company (Figure 13 System B 3). This system is specific to unlocking the value of radical innovations. The innovation is usually introduced by the managing director or another senior executive as, defined by Interviewee B1, it relies on an 
overview of the company to conceive, perceive - or appreciate - a major opportunity to develop the business. Radical innovations and their implicit capital expenditure undergo a more rigorous process to minimise potential risk.

In the example given below (Figure 13), the innovative concept captured the full interest of staff and was seamlessly adopted into the organisation. Respondents indicated this effortless adoption is the norm within the company for radical innovations owing to early involvement of all necessary parties. This early involvement addresses concerns and overcomes problems during initial stages, facilitating persuasion.

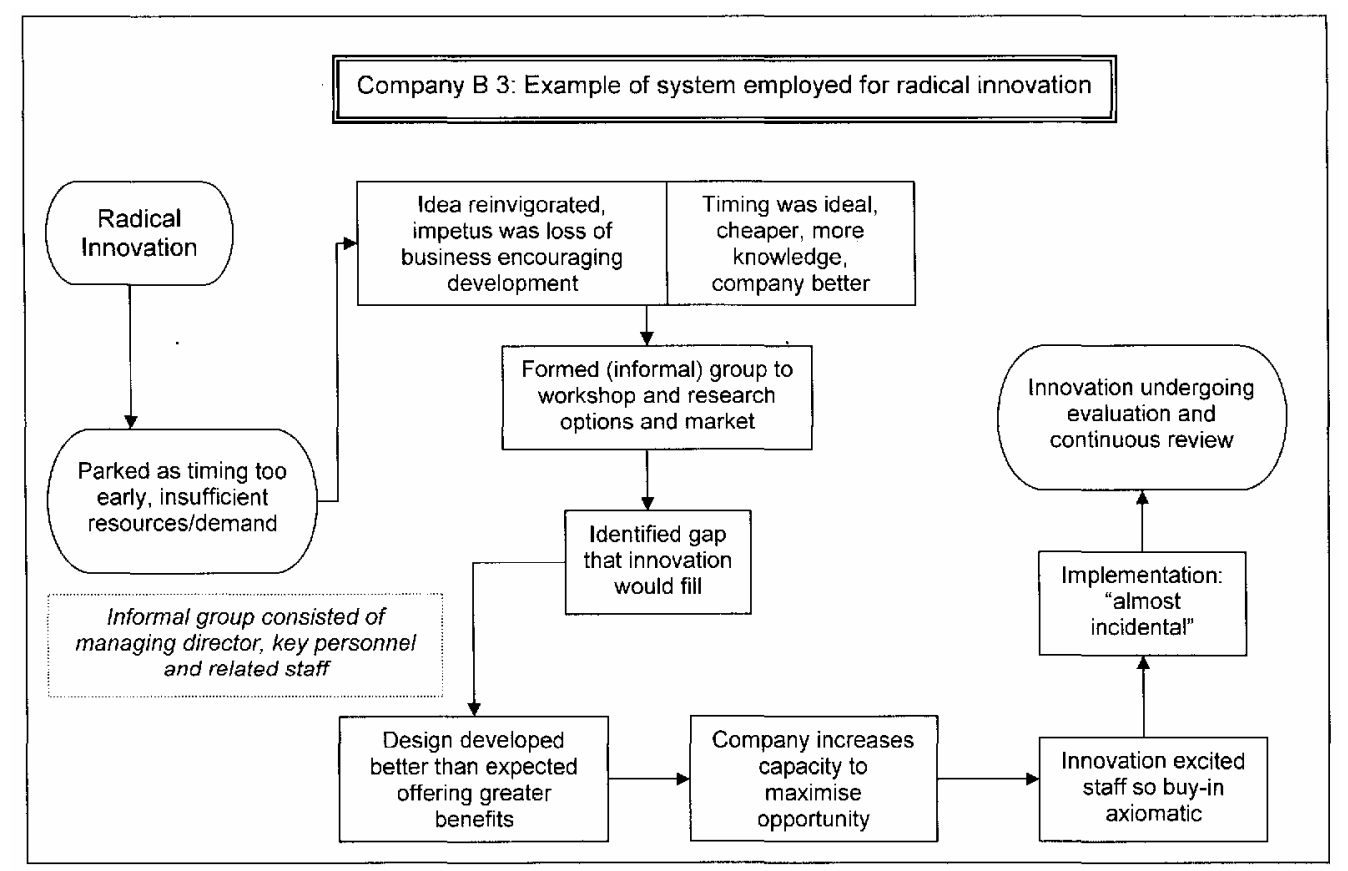

Figure 13: System B 3, example of system for radical Innovation

\section{Features of Company B's Innovation Systems}

In investigating Company B's innovation systems, specific features were apparent, such as how the company manages ideas that have merit but are not applicable at the time of presenting. Due to the rapid turnover of work in Company B's facet of the ICT industry, any unsuitable ideas are quickly terminated as inappropriate or having specific consequences that may impede work activity. There are no formal methods for capturing rejected ideas with most innovations, particularly radical innovations, relying on the memory of the generator to recall reasons for rejecting. However, owing to the nature of the Company B's business, rejected concepts are rarely resurrected as the organisation is constantly evolving negating future relevance. 
Conceiving continuous improvement innovations is a constant process assisting the organisation to meet one or more of three objectives. To paraphrase Interviewee 3, the three critical factors are:

- time (does it improve efficiency),

- quality (improving quality of work for a client),

- cost savings (for either the company or the client, or both).

The aim is to realise projects successfully through improving time scales, for the least money, at the highest quality. These three areas provide the "litmus test" (Interviewee B1) to the logic of an innovation and its application to those generating concepts and those involved in assessing innovations. Decisions are based on the soundness of idea and prompt feedback from those involved. The company considers rapid decisions normal and reflective of their business environment.

Nightly work-in-progress meetings are a regular feature of the company. These meetings provide the ideal forum for innovation discussions as a representative from each department is present to voice any concerns. This forum, with its inclusion of key personnel, also assists in refining innovations as problems and benefits can be quickly conveyed and analysed.

Once implemented innovations may experience adjustments to improve applicability to either the company or a specific activity. Similarly, a cyclic process may occur of improving an innovation, gaining feedback from relevant people and reinstigating the improved concept as knowledge of the innovation's benefits or outputs are better understood. Implementing innovation is often seen as a logical extension of activities resulting in rapid introduction. Interviewees advised that implementation is incidental in the sense that there is no requirement for purposeful or formal introduction, innovations usually being adopted as a straightforward adjustment to normal work practice. To facilitate the adoption process, use of a champion or expert is considered necessary if implementing the innovation demands a major change to work practices.

Other facets that impinge on Company B's innovation systems are the timing of the innovation, both job-specific timings and concept-to-outcome period; the type of innovation and whether the innovation is tangible or not. Considering each of these separately; firstly, timing. Incremental innovations are usually client or job specific and often terminate automatically on completion of a project. Hence, a suitable innovation is rapidly introduced and only extended to other activities if proven overall 
more efficient than regular work practices. Similarly, the time frame of an innovation being transformed into value can be reduced if the output warrants it. This means engaging stages in rapid succession or concurrently, as in investigating refinements while discussing innovation merits to accelerate realisation.

\section{Incremental Innovation and Radical Innovation}

The need for rapid decisions and rapid processing of an innovation is apparent in the volume of constant day-to-day innovations that are a function of the firm's continuous improvement philosophy. These small changes may result in what amounts to major departures from normal work practises over time. Although rare, cumulative incremental innovations have caused dramatic change and have been pivotal to the company's success.

Radical innovations inevitably require capital investment and conscious change to company operations. Due to the greater risk inherent in dramatic change, a more sophisticated system is used to realise radical innovation in Company B (Figure 11, System B 3). Interviewees advised that more meetings are necessary to address the dangerous aspects of this type of innovation and to maximise success of outcome. Or to alter or terminate the concept if unsuitable in its present state.

\section{Tangible Innovations and Intangible Innovations}

There is no difference in system application for the type of innovation, whether tangible or intangible. The only dissimilarity is that process innovations tend to more quickly implemented than product-related innovations as the latter usually require capital investment.

\section{Company B's Practices Supporting Innovation Process Management}

There are four critical areas that impact on the success of innovation in Company B: Idea generation, culture, access to decision makers and growth.

\section{Idea Generation}

The company encourages and expects all staff to submit innovative ideas. The consensus of the interviewees is that innovative concepts are welcome from anyone at any time. A comment was made that often learning from mistakes was a source of innovation inspiration. Awareness of this helps to encourage the offering of creative concepts that may have an element of risk or inspire ideas to turn a negative occurrence into a successful result. 
This attitude to creativity overcomes any reluctance to initially sharing an idea, or to embark on research or analysis of an innovation (undertaken on a casual inhouse basis) before an idea is proposed. The company operates on the principal of individual empowerment, that it is not necessary to provide elaborate explanation or financial justification if the employee believes a proposition is sound. Staff usually suggest innovations based on knowledge of the industry, the company, the client, or activity taking place.

\section{Culture}

A success factor of the company is this ability to access employees' creativity, sustaining an environment that nurtures and motivates staff to generate innovations, express them freely and, in turn, harvest these innovations. All three interviewees agreed innovation was an active function of every person in the organisation. As well, that employees embrace innovation and implement change on an ongoing basis. Respect was a word used by one interviewee to indicate the serious nature of acceptance of ideas regardless of source or concept, emphasising that there is a strong no-blame attitude which in turn reinforces openness to risk. Interviewee B1 summed it up as "Creativity and innovation are subsets of open communication and culture. Very few rules. Very limiting to have rules. Just another barrier".

The free flow of ideas, communication and ease of integration of ideas are part of the culture. As one interviewee explained “We don't have an open door policy because we don't have any doors, that is tremendously important, open plan... the way the team is structured, it is all an integral part of our innovation process." Maintaining one floor for the entire office is seen to minimise physical and psychological barriers to communication, hence, the sharing of innovative concepts. Likewise, the open office layout and the use of technology to facilitate information flow, as explained by Interviewee B3, underpins the free flow of activity, particularly important with the constant operational time pressures experienced.

The company appreciates that balancing mundane routine and inspiring creativity can be difficult. Each employee is encouraged to consider methods for improving others' roles as well as their own. Interviewee B3 advised that staff are aware of the cascade effect of innovation (how one change can have unplanned consequences), that even a minor adjustment can impact on an unexpected number of other people or projects. This awareness results in testing of innovations to facilitate adoption and to address any employee reluctance to implement. 


\section{Access to Decision Makers}

A facet of the organisational structure is the constant presence and availability of the executive team. Inter-relating with mangers is seen as vital to inspire and reassure staff of each one's importance to the operation, that every employee's contributions are appreciated and ideas encouraged. Recruitment decisions are made on the basis of an individual's suitability to fit the team, team attitude, creative thought and receptivity to adopting innovation. One interviewee commented that the management team are "Not like real bosses", referring to their approachability and depth of involvement with staff and activities. Another interviewee stated that the individuals in the management team have unique perspectives on the organisation owing to their involvement across the firm, therefore awareness of the implications (negative and positive) of innovations. This provides both the ability to appreciate the value of an innovation and recognise possible ramifications and consequences.

\section{Growth}

The company has grown rapidly over the last few years and continues to experience success and attendant development. One interviewee commented that growth has increased pressures on people further stimulating innovation. The interviewee referring to the desire of staff to work as efficiently as possible thereby reducing time involved with any single activity, more effectively juggling a larger workload, in turn supporting team members. Several interviewees saw the strong team culture as potentially threatened by growth with the ability to stay in touch with everyone being negated by sheer size and number of staff. To date, the historical culture and small size of the organisation are seen as facilitating innovation. Again, these are perceived to be at risk, compromised by the company's continuing rapid growth.

Similarly, the company's systems are increasingly under pressure owing to volume of work requiring processes that are more efficacious. However, Interviewee B1 in particular expressed reluctance to formalise any systems or work structures, preferring to maintain the casual atmosphere and work practices currently in place. Other interviewees supported this view, expressing enjoyment of the company's creative and informal structure. 


\section{Innovation Journey Influences}

Company $B$ interviewees, when presented with the list of barriers and enablers (drawn from the literature review) to the realisation of innovation in an organisation, provided a variety of responses.

\section{Company B Barriers to Innovation Realisation}

The responses to barriers (Table 6) provided some contrasting opinions between the most senior person and less senior staff. However, there is full agreement ( $N$ responses in Table 6 ) of some barriers not being present in the organisation, for instance:

- Adamantly expressed by all three was the sentiment that the use of sales targets and rewards actively discourages creativity. Such activities are perceived to introduce contradictions running counter to innovation. It was expressed that motivating and providing incentives for individuals leads to self-centred thinking and thus, compromising team orientation. This in turn reinforces potential turf wars hindering assisting others to perform better.

- Decision making by consensus. Participants' believed that employees are not afraid to make decisions. One person mentioned that everyone is quick to agree and if there is any disagreement, it is brought up straightaway with concerns being discussed. This also mitigates internal politics as each person's view is considered valid and all are encouraged to voice opinions.

- (Inappropriate) Strategic orientation. One interviewee referred to the company as seizing opportunities as they arose. Another said that by not having a real strategic plan the company is not hampered by one.

- $\quad$ Lack of support to adopt change. All three agreed that there is ample organisational support to implementing and adopting innovation. Interviewee B1 commented that "Creativity, leads to innovation, leads to growth, leads to creativity - a cyclic process".

However, opinions varied on:

- Mis-allocation of (limited) investment finances. The most senior person interviewed said that risk management was important and considered ways to mitigate risk. Whereas the other two interviewed did not see a cause for concern. 
Table 6: Company B Interviewees' responses to barriers in the firm

\begin{tabular}{|l|c|c|c|}
\hline & \multicolumn{3}{|c|}{ Interviewees } \\
\hline Barrier & B1 & B2 & B3 \\
\hline Competing management priorities. & N & N & N \\
\hline Mis-allocation of finances. & Y & N & N \\
\hline Rewards and incentives. & N & N & N \\
\hline Encouraging status quo. & N & N & N \\
\hline Management tying up critical resources better allocated. & N & N & N \\
\hline Bandwagon effect. & N & N & Y \\
\hline Fear innovation cannibalising existing products. & N & N & N \\
\hline Management inability to implement innovation. & N & N & N \\
\hline Processes that do not support change. & Y & N & Y \\
\hline Innovation introducing contradictions. & Y & N & N \\
\hline Decision making by consensus. & N & N & N \\
\hline Multiplicity of meetings to decide. & N & N & N \\
\hline Preoccupation with current activities and markets. & Y & Y & N \\
\hline Short term focus and/or an excessively internal focus. & N & Y & Y \\
\hline (Inappropriate) Strategic orientation. & N & N & N \\
\hline Internal politics. & N & N & Y \\
\hline Organisational resistance to change and/or cultural inertia. & N \\
\hline Lack of support to adopt change. & N & \\
\hline
\end{tabular}

Key: $\mathrm{Y}=\mathrm{Yes}$, agree with statement, barrier is experienced in organisation $\mathrm{N}=\mathrm{No}$, not experienced in organisation

- $\quad$ One person had experienced the Bandwagon effect, where too many people in Company $B$ want to be part of the innovation activity. Also, one interviewee made the point that delays are being experienced in introducing innovation as more people become involved in the process.

- Two interviewees felt that there are processes which do not support change (including lack of cross-division/functionality), that this barrier is increasing as the company grows in size.

- $\quad$ Similarly, two believe that there was a preoccupation with current activities and markets. Two also think that there are problems with short term focus and excessively internal focus, referring to the immediacy of daily demands causing overload, consequently preventing innovation. 
Company B Enablers to the Internal Innovation to Outcome Journey

On considering the list of enablers offered, all three interviewees agreed that most practises that support innovation outcome are present in the organisation. Identified principally was the need for a champion or sponsor to drive the process. One person commented that it is not always someone from senior management, it could be the idea generator from anywhere in the firm. If it is more relevant to another employee then the idea is handed on. With dramatic innovations, usually generated by a senior manager, this same senior person continues to drive the concept through the system.

All respondents agreed that there are no requirements in the company for:

- $\quad$ Rewarding successful innovation (cash bonuses and other incentives). Interviewees agreed that rewarding innovation is important; qualifying the different responses to this enabler by explaining that reward is simply the implementation of the innovation. The anomaly between responses is that the senior person (B1) perceives this reward to be sufficient, whilst B2 and B3 explained innovation is part of normal business practice for employees, requiring no additional incentive.

- $\quad$ Training staff to encourage innovativeness is considered redundant to the organisation. Staff are already that way inclined, any not agreeing would be misfits in the culture. The culture and the firm's encouragement of innovation are considered the only support necessary.

The above facilitators were considered by interviewees as surplus, or negating Company B's style of innovation agility. The sentiments expressed by interviewees reinforce Company B's stance of informal innovation assisted by the presence of most innovation enablers and a lack of barriers. 
Table 7: Company B Interviewees' responses to facilitators in the firm

\begin{tabular}{|l|c|c|c|}
\hline & \multicolumn{3}{|c|}{ Interviewees } \\
\hline Enabler & B1 & B2 & B3 \\
\hline Having sufficient resources to support innovations. & $\mathrm{Y}$ & $\mathrm{Y}$ & $\mathrm{Y}$ \\
\hline Access to funds innovative ideas not requiring approval. & $\mathrm{Y}$ & $\mathrm{Y}$ & $\mathrm{Y}$ \\
\hline Rewarding successful innovation. & $\mathrm{Y}$ & $\mathrm{N}$ & $\mathrm{N}$ \\
\hline Overarching team to drive process. & $\mathrm{Y}$ & $\mathrm{Y}$ & $\mathrm{Y}$ \\
\hline Having a champion management. & $\mathrm{Y}$ & $\mathrm{Y}$ & $\mathrm{Y}$ \\
\hline Training staff to encourage innovativeness. & $\mathrm{N}$ & $\mathrm{N}$ & $\mathrm{N}$ \\
\hline Drawing on customers' experience. & $\mathrm{Y}$ & $\mathrm{Y}$ & $\mathrm{Y}$ \\
\hline Customer interrelationship by backroom personnel. & $\mathrm{Y}$ & $\mathrm{Y}$ & $\mathrm{Y}$ \\
\hline Free flow of information. & $\mathrm{Y}$ & $\mathrm{Y}$ & $\mathrm{Y}$ \\
\hline Protecting innovation from bureaucratic limitations. & $\mathrm{Y}$ & $\mathrm{Y}$ & $\mathrm{Y}$ \\
\hline Setting goals for innovative achievement. & $\mathrm{N}$ & $\mathrm{N}$ & $\mathrm{N}$ \\
\hline Encouraging a long-term perspective. & $\mathrm{Y}$ & $\mathrm{Y}$ & $\mathrm{Y}$ \\
\hline Culture supportive of innovation vision. & $\mathrm{Y}$ & $\mathrm{Y}$ & $\mathrm{Y}$ \\
\hline Cultural pride in company's (innovative) achievements. & $\mathrm{Y}$ & $\mathrm{Y}$ & $\mathrm{Y}$ \\
\hline Accepting failure. & $\mathrm{Y}$ & $\mathrm{Y}$ & $\mathrm{Y}$ \\
\hline Organisational structure and cross functionality of divisions. & $\mathrm{Y}$ & $\mathrm{Y}$ & $\mathrm{Y}$ \\
\hline Individual perspective of innovation. & $\mathrm{Y}$ & $\mathrm{Y}$ & $\mathrm{Y}$ \\
\hline Innovation introduced as complementary to previous. & $\mathrm{Y}$ & $\mathrm{Y}$ \\
\hline Participative or team style of management. & $\mathrm{Y}$ & $\mathrm{Y}$ \\
\hline
\end{tabular}

Key: $Y=Y e s$, agree with statement, enabler present in organisation $\mathrm{N}=\mathrm{No}$, not experienced in organisation

\section{Innovation and System Reviews}

Both incremental innovations and innovations that are more complex may undergo re-analysis to consider further application throughout the company, in either original form or adaptation thereof. There is no formal process; revisions are considered a function of continuous improvement. That there are no intentional reviews of effectiveness of the systems to realising innovations was perceived as a probable shortcoming by one interviewee. Other expectations expressed were that if the systems are not causing concern, they must be effective. 


\section{Success of Current Systems}

Current systems are seen as $80 \%$ effective with occasional failures caused by lack of awareness of ramifications or the impact of growth. One person commented that everything can be improved, that the absence of red tape (the need to battle bureaucracy) in the company and openness of the culture facilitates the process. All three interviewees were adamant that the informality of the systems suits the company's style. However, one was of the opinion that the casualness and lack of structure may be resulting in loss of effectiveness in realising innovation, but that there was no way to know this.

Company $\mathrm{B}$ has a number of innovation-to-outcome systems to ensure innovation value realisation. The different systems cover the range of innovation types and implications accompanying them, in particular effective use of key resources (time, money, staff). The organisation enjoys the substantial presence of innovation outcome facilitators with few barriers. This is supported by an open, somewhat casual environment (both physical and psychological) and a negligible hierarchical structure. 


\section{COMPANY C}

The general manger, senior business development manager and business analyst were interviewed on the innovation systems employed by the firm. The three interviewees unanimously agreed that innovation was imperative to business today, that it is a core part of Company C's culture and responsible for its success. Additionally, one participant said that innovation kept staff stimulated, that constant improvement and being more productive are part of empowering employees and enhancing their roles. Another interviewee saw innovation as "a departure from status quo to achieve desired outcome" (Interviewee $\mathrm{C} 2$ ) referring to the opportunities available when a company is not stifled by archaic or rigid modes of operating.

\section{The Practical Innovation-to-Outcome Systems Employed}

Interviewees' described Company C's two innovation-to-outcome systems, indicating selection was dependent upon the type of innovation, the magnitude of the innovation, its impact on the company and resource demands. Although interviewees clearly outlined system stages, they advised that actual steps can occur almost simultaneously.

Firstly, the basic system (Figure 14), described by Interviewees C1 and C2. This system is frequently used as the company encourages incremental innovation to constantly improve business activities. Depending on the extent of influence and whether resources are required, the system can be a series of three simple steps, adding additional steps as the scale of the innovation (and its implications) increase. The basic system commences with an initial casual conversation with peers, manager or similar person with whom the idea generator may be comfortable talking, one interviewee describing this step as a "sanity check" (Interviewee C2). Interviewees explained that this early testing of the innovation could take the form of a brief chat in a corridor, over a desk or booth divider, referring to this as socialising the idea. Should the innovation have a very small area of influence, for example a single individual, then a casual chat with the relevant line manager either initially or subsequently, generally results in immediate decision and implementation. 
This basic system is the business as usual process, referring to its regular use to transform incremental innovations into outcome. One person (Interviewee C2) commented that the lack of a multitude of steps, experienced in larger companies, demonstrated the company's very recent entrepreneurial history.

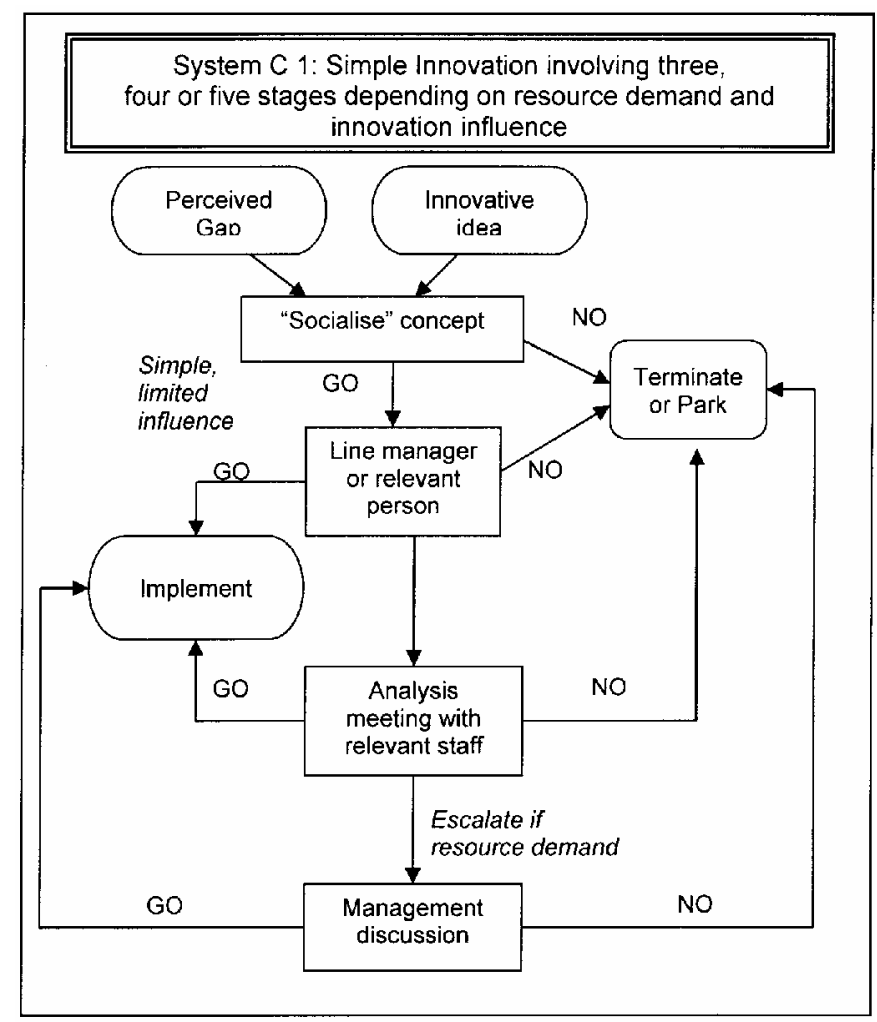

Figure 14: System C 1

\section{System C 2}

However, if major investment is indicated (often intrinsic with radical change) System C 2 is employed (Figure 15). These are high resource demanding innovations, therefore increase risk. System $\mathrm{C} 2$ is designed to balance resource use and to minimise risk with encouraging the unlocking the value of innovations. Usually this type of innovation includes dramatic change in the company's direction, re-prioritising resources.

The system is initiated on the management team (having discussed a specific situation) identifies a need or opportunity that requires capital expenditure (which may or may not include the employment of additional staff). Following, the team appoints a senior employee - whose responsibilities relate to the opportunity - to champion investigating the various options. On completing initial analysis, the champion reports back to the management team and begins formulating a business 
plan. Further analysis may be required which in turn loops back to the management team. Once the innovation and its implications are clear and the team approves the plan, it is forwarded to the board.

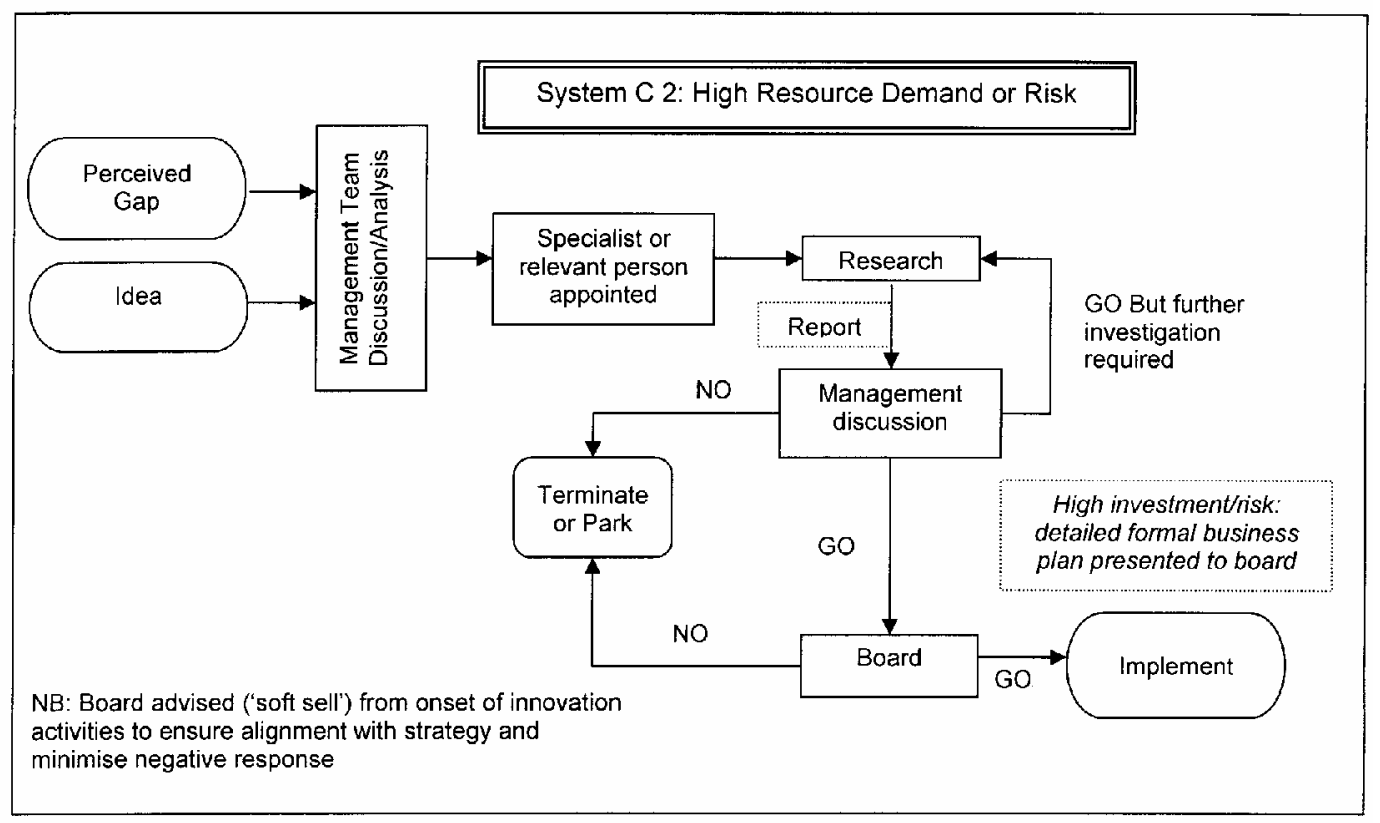

Figure 15: System C 2: Radical or resource demanding innovations

The formal business plan includes risk assessment, resource requirements and plan of action. This is presented to the board for consideration of strategic fit and approval to allocate resources. The board will have been previously advised of the innovation and related activities to ensure early suitability and minimise negative reaction. Each plan is comprehensive and contains an overview of the situation; market research (in-house); current market status and market spend, predicted market growth; framework for growth, a plan for operation development and resource requirements. The plan also outlines the multi-year strategy, step by step yearly development plan with objectives, activities and costs. A lesser version of the document may be used in System C 1 if an innovation's implications to the company warrant it.

Meetings occur in both System C 1 and System C 2 to consider anecdotal evidence together with the business plan (if one is justified) to facilitate decisions. The decision process is assisted by ensuring all relevantly effected people, or "interested parties" (Interviewee C3) are included. This gains an indication of possible responses and concerns to address before developing the innovation further. The involvement of all people in early stages relates to the company's 
expectation that innovations have team commitment. In both Systems C 1 and C 2 there may be a requirement for additional information, in which case the meeting decision is to further investigate the concept. Otherwise, if agreement is reached, the innovation is implemented with a No Go resulting in parking or terminating the innovation.

\section{System C 3: Work in progress (Figure 16)}

However, with ideas arising constantly, corresponding with company growth, increasing staff numbers and business opportunities, there is a new, formalised, system currently being developed which is intended to be normative practice (Figure 15). Interviewee $C 3$ stated that a new system was necessary to meet the complexities incumbent with company growth. As well, to address innovations that have some resource demand and influence on multiple areas of the business, the current systems not being suitable to meet the multi-division operation that now exists. The new system is in an embryonic state with stages imprecise at this time. Interviewee C3 advised that they are yet to address how radical innovation will be processed, with additional steps to be designed to facilitate this innovation type. Conversely, the small incremental innovations, these too are awaiting consideration as to how to best realise in the new process.

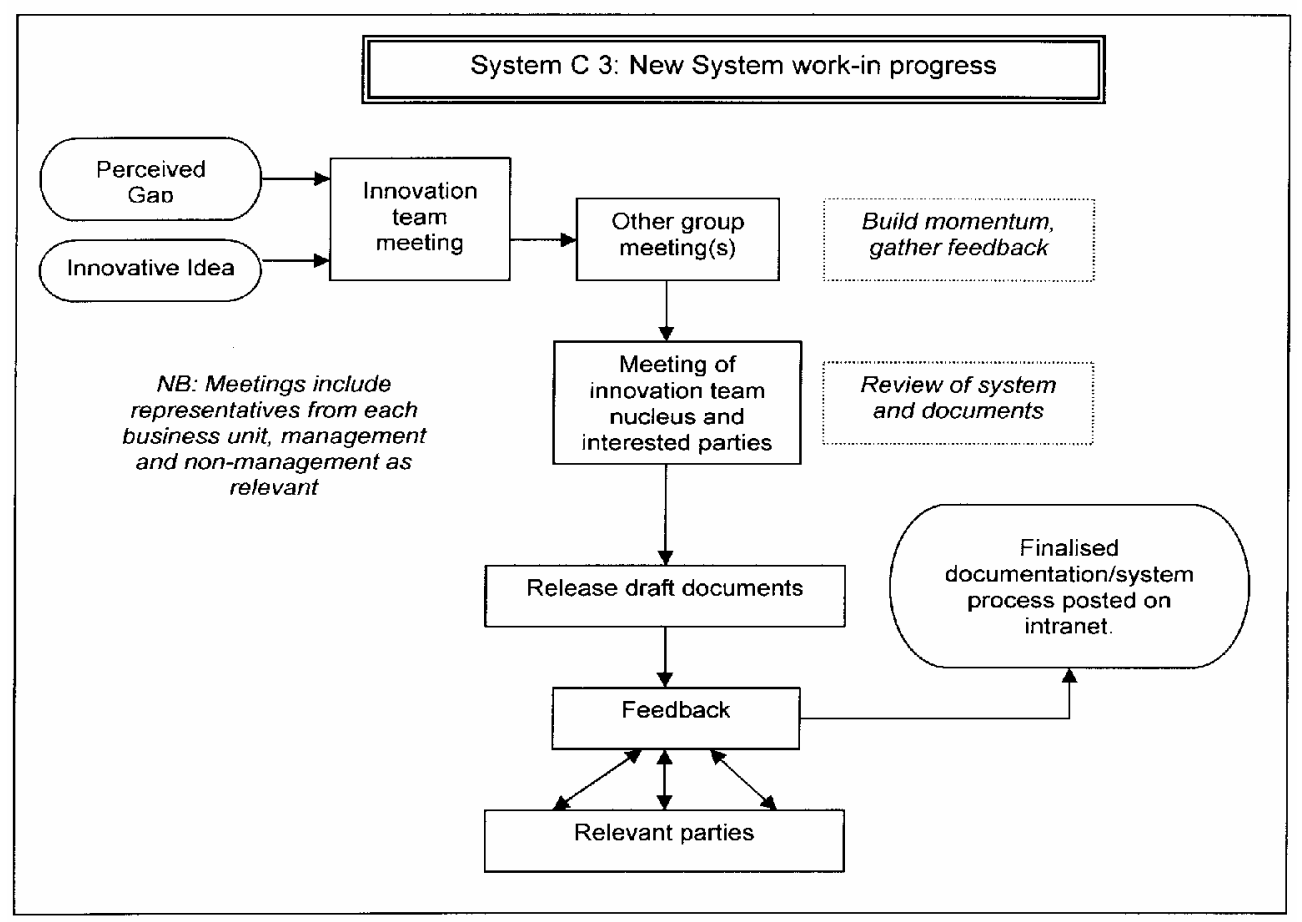

Figure 16: New Innovation System (in development) 
The first stage in this developing system is the innovation team meeting on a fortnightly basis to discuss innovation proposals. The innovation team includes people from each business unit to contribute knowledge and experience. As well, the presence of representatives of all departments facilitates buy-in of concept as the members become advocates of proceeding innovations. The next step is a further meeting, this one including management and other interested (relevant) parties. The following stages are still being formulised currently existing only as tentative outlines. This new system is primarily team based having multiple meetings to assist introducing and acceptance of approved innovations.

Each stage of the new system is being documented to complement physical actions to provide a written framework. Both the developing system and associated documents are posted on the company's intranet for general access, undergoing refinement as experience grows. As well, the design team are establishing periodical review dates to ensure future revisions of the system once active.

\section{Features of Company C's Systems}

A feature of the firm's innovation activities is the use of resources. Interviewee C1 advised that deployment of resources up to a certain limit is the responsibility of individual department managers. If additional staff or resources are required to realise a promising innovation outside of the designated amount, a cost/benefit analysis is required and agreement to additional resources approved by the managing director. The department manger is then responsible for use of the increased resource. A motivating factor in creating a new system has been the appreciation of the difficulty of securing and allocating resources. The senior team realised that there were insufficient resources for the volume of innovations being presented. Innovations most critical to the business are given priority with any outstanding innovative concepts being reassessed thereafter, if surplus resources are available.

Another feature is the importance of a champion. One person takes on the role of champion for the more complex innovation and is involved from concept through to implementation. This person is usually a key manager; however, it may be the idea generator. Interviewee $\mathrm{C} 3$ stated that the role of champion is not hierarchical, that it could be anyone as long as the person has the necessary skills. Interviewees advised that a lack of a champion will result in the innovation, despite value, inevitably terminating. Participant $\mathrm{C} 2$ preferred the word evangelist to 
champion as obsession was necessary to drive an innovation - "to impel it through inertia". (Interviewee C2)

\section{Timeliness}

Company C's aim is to realise innovations as expediently as possible. The firm does not differentiate between tangible and intangible innovations when transforming into outcome, the focus is on propensity for risk and resources, if any, required to realise. These two areas, plus the time involved, dictate which system is employed. Interviewee $\mathrm{C} 1$ explained that there is reliance on commonsense to realise when an individual is investing enough (or too much) time in an innovation. That people are already busy and have little time to dedicate to a potential new development is believed to be a significant deterrent to over allocating time to a poor innovation. The result is the awareness that some innovations simply die, not having significant impact on the business to justify further involvement. One interviewee reported that innovations stall frequently, that this is a barrier when so many innovations rely on opportunistic timing. Conversely, timing may not be right and the idea is suspended until deemed more suitable.

\section{Rejected Innovations}

Suspended or terminated innovations are not documented and rely on the initiator to remember should they become more timely or suitable. The lack of a system to capture the ideas was considered a "glaring deficiency" by one person. One interviewee advised that a feature of the new system is that suspended ideas will become a function of re-evaluation.

\section{Company C's Practices Supporting Innovation Process Management}

There were four areas having critical impact on the innovativeness of Company $\mathrm{C}$; idea generation, culture together with availability of decision makers and growth.

\section{Idea Generation}

The firm sees all employees as sources of ideas with inspiration sought within and outside the company's boundaries. Innovative ideas range from perceiving a gap and purposefully seeking solutions as a group or individually, to simply a staff person's desire to improve their work activities. As well, constant reviews of incumbent processes, systems and services generate awareness of possible innovations to improve. Interviewee C3 advised that with the new system ideas 
would belong to no one, rather a team would support concepts preventing limiting ideas and accessing a greater range of input and reinforcing the team environment.

\section{Culture}

Company $\mathrm{C}$ has a flat structure, a culture adverse to autocracy and bureaucracy. The firm encourages the open flow of communication between all staff, regardless of employees' positions. An organisation map exists but is largely ignored, one interviewee commented. Direct access to decision makers is seen to expedite innovation outcome, interviewees advised that decision makers are perceived as open and approachable by all staff. This is further aided by an open plan office, not even senior managers having individual rooms.

The company expects people to show initiative and are authorised to make decisions, unless there is major capital expenditure required. Interviewee $\mathrm{C} 1$ stated that "People are empowered to do stuff, they don't have to ask permission, sure if there is major expenditure there is a process for that... we try not to put roadblocks in their way." Consequently, concerted efforts are made to minimise barriers to innovation including reviewing and changing status quo activities. A characteristic of the culture is that there is no single right way; staff are able to challenge current operating methods, change and improve operations keeping the business moving effectively forward.

Additionally, the company prefers innovations to be accepted via encouraging an individual to consider the innovation as their initiative. Change resistance at an individual level is seen as being overcome on a regular basis through this type of buy-in together with associating innovations with previous successes. Interviewee C1 summarised it as "The best way to initiate change is for people to think it is their idea." He went on to say that adopting innovation is facilitated through

Sowing seeds... a lot of very informal or consultative type of chat going on... it is much better, in our view, to get people to do things because they think it is a good idea and they want to do it. And hopefully, even better, if you can get them to think it is their idea, then you have much more of a chance of a successful outcome. (Interviewee C1) 
As indicated by the quote, the objective of this activity is to pre-empt any negative reaction or change-resistance. The basis of the company's culture is to support activities and objectives through continuously involving all employees.

There is a strong sense of team in the company's environment with decisions and acceptance of innovation requiring team commitment and collaboration. This can lead to numerous meetings taking place to ensure adoption of a concept. One person commented that a number of people are able to veto an innovation but no single person has the ability to forward it, as decisions are reliant on group agreement. Simultaneously, innovation is seen as a way to support individuals to feel valued and realise they are important contributors to the company's ongoing success. The company fosters agility in casual (low impact) innovation decisions with the bare minimum of process to support continuous improvement.

\section{Growth}

The transition from being a small, flexible business into a more structured one is being experienced as awkward. The company is confronting the difficulty of balancing a continuing desire for growth with the demand for stability and maintenance of day-to-day business. Having multiple customers requires revision of incumbent systems to upscale, necessary to compensate for larger staff numbers and increasing pressure on resources. The company's rapid evolution has resulted in the introduction of more structured and process-orientated people necessary to support the larger company. These new staff members contrast with the hitherto creative, more entrepreneurial employees that have been with the company from inception. As well, the increasing number of staff has diluted the open communication and accessibility of information that was present in the smaller business reducing overall understanding of company activities.

Recent organisational developments include a formal induction process which previously (in the smaller company) would have been unnecessary. More structure is necessary to assist new staff performance and ongoing support. A corollary to this is that the previous more ad hoc innovation systems are being formalised to be more suitable to a larger organisation. This is as simple as introducing templates for written communication enforcing a standard of presentation, through to reviewing innovation systems. Consequently, the "pendulum is swinging" (Interviewee C2) from a more free-flowing style of operation to, in the interim, a strongly rigid 
disciplined composition. The expectation is for an amalgamation of both operating styles to occur over time.

Accessibility to busy managers is a key reason the company is developing a more structured innovation system. Constant interruption has become less tenable, compromising managers' effectiveness. However, there is extreme reluctance to not appear to discourage staff keen to discuss innovation ideas approaching managers. The creation of an innovation team to vet innovations aims to reduce this disruption.

Further, the company is experiencing greater difficulty in organising meetings for relevant personnel to discuss innovations and to prioritise resources. People are busy with daily activities and concerted effort is becoming necessary to continue an innovation culture. Yet, although formalisation is recognised as necessary, it is with reluctance that such institutionalism is required. One interviewee saw formalising the system as "closing the loop", elaborating to explain that formalisation limits creative expression and the ability to seize opportunities. This is seen by this individual as innovation-contradictory and a threat to innovation agility.

\section{Innovation Journey Influences}

The reactions to the provided list of barriers and enablers (drawn from the literature review) to innovation highlights some key differences between senior management and the two staff members interviewed.

\section{Company C Barriers to Innovation Value Realisation (Table 8)}

All three agreed that competing priorities for resources is an ongoing, axiomatic issue owing to continuing growth constantly stressing resources. Also, that there is a preoccupation with current activities and markets, particularly in regards to people being too busy with daily business, which in turn prevents innovation realisation.

The senior person (C1) saw less barriers existing in the company (indicated by the series of $\mathrm{N}$ under the $\mathrm{C} 1$ column) than did the other two who are more hands-on in the business, $\mathrm{C} 1$ perceiving only 3 of the 18 barriers to be present. $\mathrm{C} 2$, as an intrepreneurial orientated person perceives 14 barriers as present and C3, 10 barriers currently occurring, with the two agreeing on 8 barriers. For instance, innovation outcome is seen to be threatened by the desire for status quo. Similarly, organisational resistance to change together with cultural inertia is beginning to be experienced by these two interviewees. 
Table 8: Company C Interviewees' responses to barriers in the firm

\begin{tabular}{|l|c|c|c|}
\hline & \multicolumn{3}{|c|}{ Interviewees } \\
\hline Barrier & C1 & C2 & C3 \\
\hline Competing management priorities. & $\mathrm{Y}$ & $\mathrm{Y}$ & $\mathrm{Y}$ \\
\hline Mis-allocation of finances. & $\mathrm{N}$ & $\mathrm{Y}$ & $\mathrm{N}$ \\
\hline Rewards and incentives. & $\mathrm{N}$ & $\mathrm{N}$ & $\mathrm{N}$ \\
\hline Encouraging status quo. & $\mathrm{N}$ & $\mathrm{Y}$ & $\mathrm{Y}$ \\
\hline Management tying up critical resources better allocated. & $\mathrm{N}$ & $\mathrm{Y}$ & $\mathrm{N}$ \\
\hline Bandwagon effect. & $\mathrm{N}$ & $\mathrm{Y}$ & $\mathrm{Y}$ \\
\hline Fear innovation cannibalising existing products. & $\mathrm{N}$ & $\mathrm{Y}$ & $\mathrm{Y}$ \\
\hline Management inability to implement innovation. & $\mathrm{N}$ & $\mathrm{Y}$ & $\mathrm{N}$ \\
\hline Processes that do not support change. & $\mathrm{N}$ & $\mathrm{N}$ & $\mathrm{N}$ \\
\hline Innovation introducing contradictions. & $\mathrm{N}$ & $\mathrm{Y}$ & $\mathrm{Y}$ \\
\hline Decision making by consensus. & $\mathrm{Y}$ & $\mathrm{Y}$ & $\mathrm{N}$ \\
\hline Multiplicity of meetings to decide. & $\mathrm{N}$ & $\mathrm{Y}$ & $\mathrm{Y}$ \\
\hline Preoccupation with current activities and markets. & $\mathrm{Y}$ & $\mathrm{Y}$ & $\mathrm{Y}$ \\
\hline Short term focus and/or an excessively internal focus. & $\mathrm{N}$ & $\mathrm{Y}$ & $\mathrm{Y}$ \\
\hline (Inappropriate) Strategic orientation. & $\mathrm{N}$ & $\mathrm{Y}$ & $\mathrm{N}$ \\
\hline Internal politics. & $\mathrm{N}$ & $\mathrm{N}$ & $\mathrm{Y}$ \\
\hline Organisational resistance to change and/or cultural inertia. & $\mathrm{Y}$ & $\mathrm{N}$ \\
\hline Lack of support to adopt change. & $\mathrm{N}$ & $\mathrm{Y}$ \\
\hline
\end{tabular}

Key: $\mathrm{Y}=$ Yes, agree with statement, barrier is experienced in organisation $\mathrm{N}=\mathrm{No}$, not experienced in organisation

As well:

- $\quad$ Fear that innovation will cannibalise existing products. Interviewees appreciates that there has to be a necessary balance between existing and new products, services and activities resulting from innovation.

- Decision making by consensus. This is seen as presenting delays whilst helping buy-in. However, the team attitude usually results in people supporting initiative if the majority of team members agree. Similarly, multiplicity of meetings to evaluate or decide merit is being experienced as a barrier to innovation in the firm.

- Short term focus and/or an excessively internal focus: Over-focus on the company's activities and methodology is felt to be limiting the extent of 
innovation, that there needs a better balance found between internal and external focus.

One person felt that there were some instances of poor allocation of finances occurring. Similarly, management's inability to identify the best innovations in which to invest, therefore tying up critical resources better allocated to other innovative projects. Another individual identified internal politics as an issue. It was explained that problems with political manoeuvrings are increasing as growth causes the business units to blend, blurring boundaries between departments. Interviewee C2 referred to some experience with innovation saboteurs, people purposefully preventing an innovation being realised. This is thought to parallel reluctance by some staff to change.

\section{Company C: Enablers to the Internal Innovation to Outcome Journey}

There are two facilitators to innovation outcome agreed upon by all three, the need for a champion and having adequate resources. The sentiment was that often the lack of a champion will mean the demise of an innovation, particularly if there was a battle for resources. Interviewees all viewed having sufficient resources to support innovations as critical and a constant issue with limitations driving choice of innovation.

Overall, agreement regarding innovation realisation enablers in the organisation was more consistent than that of barriers. All three are in accord on the majority (13) of innovation realisation supports being present in the firm. Comments in particular were:

- Drawing on customers' experience to generate new ideas. One interviewee commented that it is important to be "looking through client's ideas and also the competitions'".

- $\quad$ One interviewee mentioned that the company can be overly internally focussed.

- Accepting failure (overcoming the fear of mistakes in being creative, being comfortable with change and conflicts). This was seen as a particularly successful attribute of the company

However, training staff to encourage innovativeness divided opinions. One saw that the company achieved this through an investment in general training experiences aimed to develop individuals' skills and abilities. Others said that 
individuals cannot be trained to be innovative, that the culture is critical to support creativity and hence, innovativeness.

Table 9: Company C Interviewees' Responses to facilitators in firm

\begin{tabular}{|l|c|c|c|}
\hline & \multicolumn{3}{|c|}{ Interviewees } \\
\hline Enabler & C1 & C2 & C3 \\
\hline Having sufficient resources to support innovations. & $\mathrm{Y}$ & $\mathrm{Y}$ & $\mathrm{Y}$ \\
\hline Access to funds innovative ideas not requiring approval. & $\mathrm{N}$ & $\mathrm{Y}$ & $\mathrm{Y}$ \\
\hline Rewarding successful innovation. & $\mathrm{N}$ & $\begin{array}{c}\text { "Grey } \\
\text { area" }\end{array}$ & $\mathrm{N}$ \\
\hline Overarching team to drive process. & $\mathrm{Y}$ & $\mathrm{Y}$ & $\mathrm{Y}$ \\
\hline Having a champion management. & $\mathrm{Y}$ & $\mathrm{Y}$ & $\mathrm{Y}$ \\
\hline Training staff to encourage innovativeness. & $\mathrm{Y}$ & $\mathrm{N}$ & $\mathrm{Y}$ \\
\hline Drawing on customers' experience. & $\mathrm{Y}$ & $\mathrm{Y}$ & $\mathrm{Y}$ \\
\hline Customer interrelationship by backroom personnel. & $\mathrm{Y}$ & $\mathrm{Y}$ & $\mathrm{D} / \mathrm{K}$ \\
\hline Free flow of information. & $\mathrm{Y}$ & $\mathrm{Y}$ & $\mathrm{Y}$ \\
\hline Protecting innovation from bureaucratic limitations. & $\mathrm{Y}$ & $\mathrm{Y}$ & $\mathrm{Y}$ \\
\hline Setting goals for innovative achievement. & $\mathrm{N}$ & $\mathrm{Y}$ & $\mathrm{Y}$ \\
\hline Encouraging a long-term perspective. & $\mathrm{N}$ & $\mathrm{Y}$ & $\mathrm{Y}$ \\
\hline Culture supportive of innovation vision. & $\mathrm{Y}$ & $\mathrm{Y}$ & $\mathrm{Y}$ \\
\hline Cultural pride in company's (innovative) achievements. & $\mathrm{Y}$ & $\mathrm{Y}$ & $\mathrm{Y}$ \\
\hline Accepting failure. & $\mathrm{Y}$ & $\mathrm{Y}$ & $\mathrm{Y}$ \\
\hline Organisational structure and cross functionality of divisions. & $\mathrm{Y}$ & $\mathrm{Y}$ & $\mathrm{Y}$ \\
\hline Individual perspective of innovation. & $\mathrm{Y}$ & $\mathrm{Y}$ & $\mathrm{Y}$ \\
\hline Innovation introduced as complementary to previous. & $\mathrm{Y}$ & $\mathrm{Y}$ \\
\hline Participative or team style of management. & $\mathrm{Y}$ & $\mathrm{Y}$ \\
\hline
\end{tabular}

Key: $\mathrm{Y}=\mathrm{Yes}$, agree with statement, enabler present in organisation $\mathrm{N}=$ No, not experienced in organisation $\mathrm{D} / \mathrm{K}=$ Don't Know

Setting goals for innovative achievement (eg. linking results with sales strategy) was considered an innate activity rather than specifically engaged. All three agreed that rewarding successful innovation (cash bonuses and other incentives) is not currently provided by the company. One interviewee did not wish to discuss this enabler, considering it a grey area and open to controversy. 


\section{Reviewing and Formalising of Company C's Innovation Systems}

Owing to the company's rapid growth and associated problems, the innovation systems are now being reviewed and adapted to become more applicable for the larger company. However, other than constant revisions of systems and activities, there are no intentional reflections on implemented innovations. Neither, as one interviewee identified, are there comparisons between expected innovation outcomes and actual conclusions. Partly this is due to the relentless growth that demands employees' attention to be on the next innovation, the next activity, constantly addressing the next pressure. As well, one interviewee expressed that the most logical person (and the last person) who would raise an issue with an implemented innovation would be the person who instigated or championed the innovation. The interviewee proposed that despite how anti-fault-laying a company may be, purposely flagging an innovation that was a mistake would be potentially foolhardy.

\section{Success of Current Systems}

Consensus among interviewees was that the company's systems to date have worked well, evidenced by the rapid growth being experienced. The company's innovativeness has resulted in the doubling of size over the last few years despite competitors' activities. It is thought by interviewees that the old innovation systems were not so much failing, rather becoming inadequate. Deficiencies in the incumbent processes were overcome currently through application of hard work.

To summarise Company C's innovation outcome activities, the impetus of growth is forcing review of current innovation systems. The systems have been successful to date but are now under pressure due to the larger organisation. A major challenge to the company is how to combine the entrepreneurial spirit and style of operation with the needed formality and processes required for the transitioning company. In other words, how to retain an innovation focus whilst managing resources effectively. 


\section{CROSS CASE SYNTHESIS}

This section compares the innovation journey activities of the three companies, the variations, nuances and similarities of the systems. This cross-case comparison assists in comprehending the overall activities that lead to actualising innovations. Firstly, to confirm the attitude of the case study companies to the status of innovation in business today. Results emphasise that innovation is paramount to the three businesses with interviewees' unanimous and strenuous responses attesting to its importance. Each of the businesses has experienced rapid growth due to their focus on effective realisation of innovation and bias for constant improvement (a function of incremental innovation) in particular.

\section{Case Study Companies' Systems}

In each case, the basic systems companies employ share strong likeness. These basic systems are then maintained as the core sequence for the more complex systems with variations in the stages reflecting the individual characteristics of each business. For example, System B 1 (Figure 10, p. 73) includes nightly production meetings at which innovations are aired. Similar regular meetings are not normal for Companies $A$ and $C$, yet these latter two firms provide forums (arranged or casual meetings) for sharing innovations at early stages. Similarly, Company A has a system (Figure 6, System A 2, p. 57) specifically designed to handle the range of innovations submitted via the company's intranet, not present in Companies B and C. Company C's Systems C 1 (Figure 14, p. 86) and C 2 (Figure 15, p. 87) collapse a number of the other businesses' separate system events into fewer steps.

All of the systems commence with the trialling of the concept with others. Referred to by various interviewees as socialising, filtering, sanity checking, or the sniff test, it is the sharing of the idea with trusted associates. If the concept doesn't gain approval at this embryonic stage, the concept goes no further. Or the idea generator may reflect on the informal advice given and re-introduce the idea incorporating the comments received, repeating this initial step.

\section{Deviations}

Distinct differences occur in the atypical system designed by Company A for stimulating innovation in the IT department, and their use of Cooper's Stage Gate system (2005b) as the framework for System A 3 (Figure 7, p. 58) to streamline resource allocation. The size of Company $A$ is reflected in its larger number of 
systems and specific IT department-orientated system necessitated by the demands incumbent with a mid-sized organisation. Likewise, Company C's purposeful development of a system to formalise their internal innovation process (Figure 16, p.88) which is strongly team orientated, requiring frequent meetings to discuss innovations. However, the structure of the final system may or may not consist of the steps currently proposed. Company B's fierce determination to prevent formalisation of any of its systems departs from the other two companies' drive to establish structured systems.

\section{Radical Innovation Systems}

The systems developed for radical innovations again display commonality with few overall variances. These more elaborate systems supplement the basic ones with additional steps addressing propensity to risk of such innovations. However, differences are that Companies $A$ and $C$ prepare reports which are ultimately presented to a board, whereas Company $B$ lacks formal documentation, nor does it have a board. Yet all three engage similar stages including senior level meetings, analysis of costs and benefits and implications, examination of resource requirements and circular feedback activities. All activities aimed to limit risk and ensure best use of resources.

The range of systems can be collated (Table 10) with distinct variations separated out, reinforcing system similarities (see Appendix $E$ for basic systems and Appendix $F$ for radical systems displayed together). The system not included in Table 10 is Company A's Atypical System, as it is unique and characteristic to the company's realm of activities (Figure 9, System A 5, p. 60).

Table 10: Compilation of Case Study Companies' Systems

\begin{tabular}{c|c|c}
\hline Company & System & Variation \\
\hline A & A 1 & Ideas tree for lodging concepts \\
\hline & A 2 & Ideas lodged on intranet \\
\hline & A 3 & Cooper's Stage Gate basis \\
\hline B & A 4 (Radical) & Preparation of business plan \\
& B 1 & Nightly production meeting. \\
& B 2 & Reference to sponsor or expert. \\
\hline & B 3 (Radical) & Dofinite cyclical feedback process \\
\hline C & C 1 & Preparation of business plan \\
\hline & C 2 (Radical) & (System work in progress)
\end{tabular}




\section{Practises Supporting Innovation Realisation}

Companies A, B and C have similar cultures and attitudes to innovation underpinning their realising of innovation value. Similarly too, the companies have concerns that operational structures are under pressure as a result of their innovation accomplishments, in turn putting stress on these self-same systems.

\section{Innovation Journey Influences}

Responses to barriers and facilitators varied with each company and between respondents. Table 11 displays the collective responses of the nine interviewees to the presence of barriers in each company. Reviewing combined comments, only one barrier was perceived as contrary to innovation activities by all participants: The use of incentives to promote innovation. Specific remuneration for supporting innovations is seen as opposing each company's culture. Reference was also made by interviewees to the difficulty of identifying individuals in team-focused environments and the potential for offence in singling out employees.

Another barrier identified by the majority is the conflict between current activities and innovativeness with only one person stated that distraction of day-today work was not a barrier to innovation agility. Similarly, a single interviewee expressed concern that the strategy of the company was innovation inappropriate; another, that management was ineffective at implementing innovation. Further, according to responses, both Companies $A$ and $C$ have issues with management's ability to best allocate resources. The two companies also had the senior person express a different opinion to subordinates regarding encouraging status quo preventing innovation.

In general, the three senior personnel (A1, B1, and $\mathrm{C} 1)$ of each company responded similarly to each other, for instance believing that there is no concern with the company being overly short-term focussed. The three senior people differed to each other on five of the 18 barriers. Company $\mathrm{C}$ displays the greatest difference of opinion between the senior person and subordinates with six instances of contrasting opinion, Companies $\mathrm{A}$ and $\mathrm{B}$ each having only three instances. Company $\mathrm{C}$ also has the largest number of barriers felt to be present with 27 positive responses to their existence in the organisation preventing innovation outcome, with Company $A$ identifying 18 and Company B reporting 10 out of the possible 54. 
Table 11: Combined Companies' Barriers to Innovation Outcome

\begin{tabular}{|c|c|c|c|c|c|c|c|c|c|}
\hline Barrier & A1 & A2 & A3 & B1 & B2 & B3 & C1 & $\mathrm{C2}$ & C3 \\
\hline Competing management priorities. & Y & Y & $Y$ & $\mathrm{~N}$ & $\mathrm{~N}$ & $\mathrm{~N}$ & $Y$ & $Y$ & $Y$ \\
\hline Mis-allocation of finances. & $\mathrm{N}$ & $\mathrm{N}$ & $\mathrm{N}$ & Y & $\mathrm{N}$ & $\mathrm{N}$ & $\mathrm{N}$ & Y & $\mathrm{N}$ \\
\hline Rewards and incentives. & $\mathrm{N}$ & $\mathrm{N}$ & $\mathrm{N}$ & $\mathrm{N}$ & $\mathrm{N}$ & $\mathrm{N}$ & $\mathrm{N}$ & $\mathrm{N}$ & $\mathrm{N}$ \\
\hline Encouraging status quo. & $\mathrm{N}$ & Y & Y & $\mathrm{N}$ & $\mathrm{N}$ & $\mathrm{N}$ & $\mathrm{N}$ & Y & Y \\
\hline $\begin{array}{l}\text { Management tying up critical } \\
\text { resources better allocated. }\end{array}$ & $\mathrm{N}$ & Y & Y & $\mathrm{N}$ & $\mathrm{N}$ & $\mathrm{N}$ & $\mathrm{N}$ & Y & $\mathrm{N}$ \\
\hline Bandwagon effect. & $\mathrm{N}$ & $\mathrm{N}$ & $\mathrm{N}$ & $\mathrm{N}$ & $\mathrm{N}$ & Y & $\mathrm{N}$ & Y & Y \\
\hline $\begin{array}{l}\text { Fear innovation cannibalising existing } \\
\text { products. }\end{array}$ & $\mathrm{N}$ & $\mathrm{N}$ & $\mathrm{N}$ & $\mathrm{N}$ & $\mathrm{N}$ & $\mathrm{N}$ & $\mathrm{N}$ & $\mathrm{Y}$ & Y \\
\hline $\begin{array}{l}\text { Management inability to implement } \\
\text { innovation. }\end{array}$ & $\mathrm{N}$ & $\mathrm{N}$ & $\mathrm{N}$ & $\mathrm{N}$ & $\mathrm{N}$ & $\mathrm{N}$ & $\mathrm{N}$ & Y & $\mathrm{N}$ \\
\hline Processes that do not support change. & $\mathrm{N}$ & Y & $\mathrm{N}$ & Y & $\mathrm{N}$ & Y & $\mathrm{N}$ & $\mathrm{N}$ & $\mathrm{N}$ \\
\hline Innovation introducing contradictions. & Y & Y & $\mathrm{N}$ & $\mathrm{Y}$ & $\mathrm{N}$ & $\mathrm{N}$ & $\mathrm{N}$ & Y & Y \\
\hline Decision making by consensus. & $\mathrm{N}$ & Y & $\mathrm{N}$ & $\mathrm{N}$ & $\mathrm{N}$ & $\mathrm{N}$ & Y & Y & $\mathrm{N}$ \\
\hline Multiplicity of meetings to decide. & $\mathrm{N}$ & Y & $\mathrm{N}$ & $\mathrm{N}$ & $\mathrm{N}$ & $\mathrm{N}$ & $\mathrm{N}$ & Y & Y \\
\hline $\begin{array}{l}\text { Preoccupation with current activities } \\
\text { and markets. }\end{array}$ & Y & Y & $\mathrm{Y}$ & $\mathrm{Y}$ & $\mathrm{Y}$ & $\mathrm{N}$ & Y & $\mathrm{Y}$ & Y \\
\hline $\begin{array}{l}\text { Short term focus and/or an excessively } \\
\text { internal focus. }\end{array}$ & $\mathrm{N}$ & $\mathrm{N}$ & $\mathrm{N}$ & $\mathrm{N}$ & $\mathrm{Y}$ & Y & $\mathrm{N}$ & Y & Y \\
\hline (Inappropriate) Strategic orientation. & $\mathrm{N}$ & $\mathrm{N}$ & $\mathrm{N}$ & $\mathrm{N}$ & $\mathrm{N}$ & $\mathrm{N}$ & $\mathrm{N}$ & $\mathrm{Y}$ & $\mathrm{N}$ \\
\hline Internal politics. & $\mathrm{N}$ & $\mathrm{Y}$ & Y & $\mathrm{N}$ & $\mathrm{N}$ & $\mathrm{N}$ & $\mathrm{N}$ & $\mathrm{N}$ & Y \\
\hline $\begin{array}{l}\text { Organisational resistance to change } \\
\text { and/or cultural inertia. }\end{array}$ & $\mathrm{N}$ & $\mathrm{N}$ & $\mathrm{N}$ & $\mathrm{N}$ & $\mathrm{N}$ & Y & $\mathrm{N}$ & Y & $\mathrm{N}$ \\
\hline Lack of support to adopt change. & $\mathrm{N}$ & $\mathrm{N}$ & Y & $\mathrm{N}$ & $\mathrm{N}$ & $\mathrm{N}$ & $\mathrm{N}$ & $\mathrm{N}$ & Y \\
\hline
\end{tabular}

Key: $Y=Y e s$, agree with statement, barrier is experienced in organisation $\mathrm{N}=\mathrm{No}$, not experienced in organisation

Barriers listed firstly under Resources, Processes, Values, delineated by a double line. 
Overall, Company $\mathrm{C}$ had the most issues with barriers, reflecting the difficulties the company is experiencing adjusting from a small entrepreneurial organisation to an established larger business. However, in general barriers were not considered overly dominant in preventing innovation realisation success by interviewees in all three companies.

\section{Supports to Innovation Outcome Success}

Combining the results of enablers (Table 12) to innovation provides more consistency of response. Of particular note is the agreement by all participants to 13 of the 19 facilitators being present in each company. The enabler: Rewarding, training and setting of goals for staff to encourage innovation, caused differences of opinion in two of the three companies with the consensus being that this facilitator was not relevant.

Senior staff (A1, B1, C1) shared greater alignment with sub-ordinates regarding the presence of enablers compared to the existence of barriers in the previous table (Table 11). In Company $\mathrm{B}$, the senior person (B1) viewed rewarding innovation as a function of the idea being introduced into the firm and acknowledging the source, considering this sufficient reward. This explanation differed from other Yes responses in that Company $A$ has some practices in place to recognise innovation, specifically the annual award system for the IT division. Otherwise, the presence of rewards is similar (in other words non-existent) with no specific bonuses paid or incentives to promote innovation by the three companies. The companies all have an expectation that generating innovations is a component of each employee's role; therefore, there is no requirement for additional rewards or incentives.

Company B's interviewees uniformly reported that setting goals for innovation was irrelevant to the company as being innovative was fundamental to operating. Company A has instituted such activities, whereas the senior executive of Company $C$ did not think the firm had which differed from subordinates' comments. Interestingly, Company C's senior person considered there were enablers lacking, that staff ( $\mathrm{C} 2$ and $\mathrm{C} 3$ ) believe are present. In all, Company A registered two negatives to the existence of a particular enabler in the firm, Company $B$ eight and Company $\mathrm{C}$ five, out of possible 57 responses. 
Table 12: Combined Companies' Facilitators to Innovation Outcome

\begin{tabular}{|c|c|c|c|c|c|c|c|c|c|}
\hline Enabler & A1 & A2 & A3 & B1 & B2 & B3 & C1 & C2 & C3 \\
\hline $\begin{array}{l}\text { Having sufficient resources to support } \\
\text { innovations. }\end{array}$ & Y & $\mathrm{Y}$ & Y & Y & Y & Y & $\mathrm{Y}$ & $\mathrm{Y}$ & Y \\
\hline $\begin{array}{l}\text { Access to funds innovative ideas not } \\
\text { requiring approval. }\end{array}$ & Y & Y & Y & Y & $\mathrm{Y}$ & Y & $\mathrm{N}$ & $\mathrm{Y}$ & Y \\
\hline Rewarding successful innovation. & Y & Y & Y & Y & $\mathrm{N}$ & $\mathrm{N}$ & $\mathrm{N}$ & \# & $\mathrm{N}$ \\
\hline Overarching team to drive process. & $\mathrm{Y}$ & $\mathrm{Y}$ & Y & Y & $\mathrm{Y}$ & Y & $\mathrm{Y}$ & $\mathrm{Y}$ & Y \\
\hline Having a champion management. & $\mathrm{Y}$ & Y & Y & Y & Y & Y & Y & Y & Y \\
\hline $\begin{array}{l}\text { Training staff to encourage } \\
\text { innovativeness. }\end{array}$ & $\mathrm{N}$ & Y & Y & $\mathrm{N}$ & $\mathrm{N}$ & $\mathrm{N}$ & $\mathrm{Y}$ & $\mathrm{N}$ & Y \\
\hline Drawing on customers' experience. & Y & Y & Y & Y & Y & Y & Y & $\mathrm{Y}$ & Y \\
\hline $\begin{array}{l}\text { Customer interrelationship by } \\
\text { backroom personnel. }\end{array}$ & $\mathrm{Y}$ & $\mathrm{N}$ & Y & Y & Y & Y & $\mathrm{Y}$ & Y & $\begin{array}{l}\mathrm{D} / \\
\mathrm{K}\end{array}$ \\
\hline Free flow of information. & Y & $\mathrm{Y}$ & Y & Y & $\mathrm{Y}$ & $\mathrm{Y}$ & $\mathrm{Y}$ & $\mathrm{Y}$ & Y \\
\hline $\begin{array}{l}\text { Protecting innovation from bureaucratic } \\
\text { limitations. }\end{array}$ & Y & $\mathrm{Y}$ & Y & Y & $\mathrm{Y}$ & Y & $\mathrm{Y}$ & $\mathrm{Y}$ & Y \\
\hline $\begin{array}{l}\text { Setting goals for innovative } \\
\text { achievement. }\end{array}$ & Y & Y & Y & $\mathrm{N}$ & $\mathrm{N}$ & $\mathrm{N}$ & $\mathrm{N}$ & $\mathrm{Y}$ & $\mathrm{Y}$ \\
\hline Encouraging a long-term perspective. & Y & Y & Y & Y & Y & Y & $\mathrm{N}$ & Y & Y \\
\hline Culture supportive of innovation vision. & Y & Y & Y & Y & Y & Y & Y & Y & Y \\
\hline $\begin{array}{l}\text { Cultural pride in company's } \\
\text { (innovative) achievements. }\end{array}$ & Y & Y & Y & $\mathrm{Y}$ & $\mathrm{Y}$ & $\mathrm{Y}$ & Y & $\mathrm{Y}$ & $\mathrm{Y}$ \\
\hline Accepting failure. & Y & Y & Y & Y & Y & Y & Y & Y & $\mathrm{Y}$ \\
\hline $\begin{array}{l}\text { Organisational structure and cross } \\
\text { functionality of divisions. }\end{array}$ & Y & Y & Y & Y & Y & $\mathrm{Y}$ & Y & Y & $\mathrm{Y}$ \\
\hline Individual perspective of innovation. & $\mathrm{Y}$ & Y & Y & Y & Y & Y & $\mathrm{Y}$ & Y & $\mathrm{Y}$ \\
\hline $\begin{array}{l}\text { Innovation introduced as } \\
\text { complementary to previous. }\end{array}$ & Y & Y & Y & Y & $\mathrm{Y}$ & Y & Y & $\mathrm{Y}$ & Y \\
\hline $\begin{array}{l}\text { Participative or team style of } \\
\text { management. }\end{array}$ & Y & $\mathrm{Y}$ & Y & Y & Y & Y & Y & $\mathrm{Y}$ & $\mathrm{Y}$ \\
\hline
\end{tabular}


Key: $Y=Y e s$, agree with statement, enabler present in organisation

$\mathrm{N}=\mathrm{No}$, not experienced in organisation

$\mathrm{D} / \mathrm{K}=$ Don't Know

\# = One participant did not wish to discuss this seeing it a grey area

Enablers are listed firstly under Resources, Processes, Values, delineated by a double line.

\section{Innovation and System Reviews}

Responses to this enquiry ranged from appreciating that such reviews could be valuable should time permit it, to comments that such an exercise was simply unnecessary. The only exception is the current development of the new system in Company C. However, the final appearance of this system is not known but part of the development process includes investigating what is not currently effectively working in existing systems.

\section{Success of Current Systems}

The sentiment regarding current systems' success is summed by the following quote "Don't know what you don't know" (Interview A1). This refers to innovation output as seen as satisfactory and the systems generally effective as the alternative is unknowable. The attitude was that the systems have been working well, particularly given circumstances (time and work pressure, balance between daily business and growth). In Company $\mathrm{A}$ and $\mathrm{C}$ improvements are being made restructuring and formalising systems to become more applicable to the now larger businesses, changes that are still being formulated. Overall, it was felt that if the systems were not working well the companies would not be as successful as they are.

\section{Summary of Case Study Results}

The three companies share a number of similarities in the systems each has developed for realising innovation outcome. Variations and nuances exist reflecting individual company interpretations and intrinsic demands relative to the nature of each business. Overall, features and characteristics of the companies are generally consistent with many commonalities of organisation and culture. Similarly, the overall presence of influences on the systems appears similar.

These barriers and supports to innovation outcome display some differences in opinion between members of each group with personnel not always agreeing with the most senior staff member. Supports to the innovation journey appear to be strong in all three companies. However, there is awareness of the presence of barriers needing to be overcome, which is being addressed through identifying and formalising of some systems. 


\section{Chapter 5 \\ DISCUSSION}

\section{Chapter Outline}

This research investigates managing the internal journey undertaken by innovation to successfully unlock its value. This chapter discusses the results detailed in the previous section and presents key findings. The results are evaluated in conjunction with the focus of the study in order to better understand the features and influences on the innovation systems. Various models and references are reviewed complementary to this discussion drawing on the literature search in Chapter 2, in particular Rogers' Innovation Decision Process model (2003). Conclusions of the study are presented in the next section.

The Discussion section is structured in relation to the research focus summarised by the research question "How can the process of managing innovation from inception to outcome be modelled?" and the research objectives:

1. To model the path of innovation through the event sequence from inception to completion or abandonment.

2. To identify from examination of these innovation models any patterns or common practices that typify the management of transforming innovation.

3. To determine any patterns and practices hindering the process reaching a successful outcome.

4. To determine any patterns and practices associated with successful, value-creating outcomes.

5. To develop a framework that will provide useful as a basis for guiding best practice in innovation management

\section{Significant Findings}

\section{Modelling Innovation Systems}

In regard to the first objective, results reveal that the case study companies have similar types of innovation systems for realising innovation and that there are patterns to the processes. A cursory inspection of the various systems indicates that there are disparities, with systems having individual variations distinct to each organisation. However, overall similarities dominate. Findings point to the innovation journey being a simplistic system at the basic level which operates to 
realise incremental innovation, while also providing the foundations for further systems. System complexity increases relative to the demands and risks inherent with the nature of an innovation. Supplementary stages in the more complex systems aim at protecting companies from unacceptable levels of risk and poor use of scarce resources. Radical innovations must be closely analysed as they may provide major advantages while also presenting implicit dangers to ongoing company existence.

The basic systems developed by the companies may be exactly that, basic, for a number of reasons. Encouraging expediency and ease in realising incremental innovations motivates a similarly quick, easy system that in turn inspires further improvements. As well, the lack of serious ramifications and limited influence of these small innovations imply little need for safety measures. The companies appreciate the overall importance of the continual improvement of operational systems, processes and services these innovations represent. These improvements help to differentiate each company's offering leading to competitive advantages. As such, the above activities reinforce the views posited by Jones (2002b), Nonaka and Takeuchi (1995), Sharma (1999) and Thornberry (2001) that competitive advantages and incremental improvement are integrally linked.

Often the continuous improvement innovations are intangible with implementation simply an adjustment to current activities. Tangible innovations, being resource-dependent, entail an increase in the number of system steps to realise. Similarly, intangible innovations that suggest a predisposition to risk require more stages to ensure innovation benefits and implications are well considered. Consequently, the tangibility of an innovation is not necessarily an indicator of which system to employ. This supports Tidd and Hull's (2003) findings that there is little differentiation between intangible and tangible innovations and the overall type of system used to realise. Conversely, findings indicate that actual use of a new product development model appears to be unsuitable for intangible innovations. New product models may be too rigid and dependently focussed on tangible-based activities to readily translate to intangible innovations. As well, the overt NPD system focus on commercialisation (as the output of a tangible innovation) may proffer steps inappropriate to innovations that have alternative outcomes (for instance work system changes). 
With regard to time-limited radical innovation opportunities, regardless of risk, the companies over-ride established systems for expediency. Timeliness of the innovation to, for example, seize market share owing to a competitor's mistake can be rushed through using a minimum of steps and is a demonstration of each company's innovation agility. Innovation agility is necessary to ensure timeliness and prevent loss of opportunity due to the delays that the more usual, safetyorientated time-consuming systems incur.

Results reveal companies run a variety of systems concurrently. The systems share resemblances with the overall objectives of minimising risk, maximising innovation outcome and ensuring best use of resources underpinning processes. The companies shift from the basic version to ones that are more complex if implications indicate the need for greater safeguards. However, the individual stages can operate in tandem, not always consecutively. Schroeder et al (1986) and Pavitt (2003) state that locked in steps are unrealistic. This study's findings are that each step generally flows into the next without waiting for a previous stage to complete. Additionally, flexibility of the systems is apparent for instance, in the number of meetings or number of participants involved in the decision stage. Flexibility is necessary in order to best realise innovations despite their random and novel nature (Williams, 1999).

However, that the systems simultaneously can inter-lap and run sequentially is counter to Van de Ven et al (1999), Schroeder et al (1986) and Pavitt's (2003) propositions that innovation systems are not linear. However, the linear progression of the companies' practical systems is vague rather than rigid. The more detailed systems include additional steps depending upon the innovation's complexity or riskpropensity of the concept. Despite including supplementary steps, the more elaborate innovation systems maintain a primarily linear progression of steps. The straightforwardness of systems appears to be due to companies desiring the least complicated processes to realise innovation value. This implies that truly intricate systems are not sustainable in the innovation agile, rapidly growing organisations.

\section{Differences in Interviewee' Responses}

A point to note is that variations in interviewees' responses may be due to several reasons. Some companies encourage frank conversation (as do some individuals) leading to better overall awareness of organisational activities. Also, a senior person has a greater overview of a company and therefore perceives 
activities more strategically. Less senior people are more likely to be directly involved in daily business than what is meant to happen from a strategic viewpoint. These different perspectives may well provide contrasting views on innovation activities within the same organisation. Additionally, there are most likely different interpretations of what constitutes innovation and the influences on innovation realisation.

\section{Innovation Management Practices}

Objective Two refers to each company's practices supporting innovation realisation. Patterns are evident as repeatedly idea generation, culture and access to decision makers feature as key components to unlocking innovation value. These features relate to Kaplan and Norton (2000) and VanGundy's (2005) view that innovation realisation success can be attributed to a business's culture as supportive of innovation. As such, the companies provide a culture receptive to all sources of innovative concepts (Senge, 1992) and free expression of these concepts, while operating under the anticipation that staff will generate innovative ideas for improving activities.

\section{Culture.}

The coherence of culture and the related access to decision makers emphasises the criticalness of sustaining an open environment, with communication free flowing and inclusive of all employees. Exemplified by the case study companies, the imposition of overt hierarchal or rigid structures and bureaucracy appears to be an anathema to innovation-successful companies (Boston Consulting Group, 2005; Jones, 2002a; Srivastava \& Gnyawali, 2006; Williams, 1999). Additionally, such organisational structures are contrary to innovation agility as evidenced by the barriers listed in Table 2 (p. 28). Analysis of the case study companies reinforces the necessity of limiting any formalising of the creative operating environment or introducing change-adverse structures. Preventing overly strict enterprises and supporting open, less formal environments are necessary complements to innovation realisation (Jones, 2002b). Change-resistant firms axiomatically will not be as innovation-outcome successful as change is a necessary part of both stimulating and implementing innovation. As Hamel and Getz (2004), Kanter (1982; 2002), Pinchot (1987), Sheppard and Canning (2006) all stipulate, change and innovation are subsets of each other. 
Commonality of cultures and company philosophies are illustrated in the receptivity of innovative ideas throughout the organisations, with innovation encouraged and expected as part of employee function by the three companies. This expectation is conjoint with the drive by the firms for continuous improvement and corresponding incremental innovations. Comparable too is the operating of teams as part of the organisational structure. An idea generator often first tests an innovative concept with team members, whether peer or senior level. This is consistent across the companies, all employee levels and departments. The innovation culture attitude of the three organisations extends to continual reviews and analysis of existing processes looking at ways to automate activities and increase efficiencies, as posited by Williams (1999). That each case study company has a continuous improvement culture correlates with their successful bias for innovation development and realisation. This reinforces McAdam, Stevenson and Armstrong's (2000) proposition that one builds the foundation for the other.

\section{Organisational Structure.}

Evidence from the three companies studied shows similarity as well of the organisational structure to be consistently flat with little hierarchy. This runs counter to the Boston Consulting Group survey (2005) that all types of organisation structures are found in innovative companies. Congruent also among the case study companies is an open door philosophy with open plan office environments limiting physical and psychological boundaries, thus reinforcing Jones (2002b) and Srivastava and Gnyawali's views (2006) that the openness of company environment is critical to innovation. Senior staff members are cognisant of the importance of limiting boundaries to innovation, supporting the free flowing of information and communication to maintain innovation activities. This desire to limit psychological and physical boundaries was explained by the senior participants interviewed as being behind the openness of companies' work environments including direct access to executives by all staff (Jones, 2002a; Williams, 1999).

\section{Growth}

The necessity of maintaining a creative-orientated culture (as a subsidiary of innovation) is contrary to formalisation (Amabile, 1998). Yet growth of the three companies is proving to increase the cumbersome aspects of actualising innovation compromising this previously automatic part of each organisation's entrepreneurial innovative climate. Growth then, is resulting in some formalising of innovation systems (Greiner, 1998) and the allocating of resources in two of the companies. 
Adapting Cooper's (2005b) new product development system is proving effective for one company in assisting allocating resources. One case study company, determined to maintain its strictly informal culture, is currently rejecting any degree of formalising and is considering alternative ways to address growth-related concerns. Results imply that managing the two states is a challenge to companies and relates to Amabile's (1998) view that this is often the case. Reference to the works of Senge (1992) on the learning organisation and Amabile's (1998) and Williams (1999) (among others) studies on sustaining creativity in business and overcoming barriers offer methods that may be worthwhile in achieving some equilibrium.

As well, the conundrum of balancing daily operations with innovation imperatives is challenging each company to re-consider operational structures. As well, management now need to consider how to effectively and deliberately stimulate innovation to sustain innovation activities. The serving of increasing numbers of customers is dominating employees' attention, compromising innovation opportunities. Hence to sustain growth initiatives requires the oxymoron of formalising innovation, or ordering chaos, (Tidd et al., 1998). Establishing a structured process aims to more effectively use resources (for example, staff time) and therefore realise innovation more effectively, actions which impose structure contrarily can limit creativity (Amabile, 1998).

That these features are a recurrent theme of the companies may relate to the choice of industry or the businesses themselves, rather than being consistent to all innovative companies. However, the differences in business activities and structures of the three organisations tend to mitigate this. Rather it is likely that this theme is representative of innovative organisations particularly at this level of Griener's (1998) first stage to second stage transitional position. The affects of growth on each company echoes Griener's (1998) early phase of shifting from creativity as a young company's organisational basis, to defining structure and formalising processes.

Strategy

This study's Adaptation of Mintzberg's (1994) strategy model (Figure 1, p. 14) replicates findings that innovative ideas may originate from numerous sources. Ideas are drawn from expected sources (resulting from purposeful planning) through to random suggestions from employees. Employee suggestions often result in cost savings or greater efficiencies garnered from direct work experience. Although the generating of innovations is assumed as part of employment and culture, actual 
occurrence is haphazard. These concepts, on passing the embryonic sniff test, are furthered shared engaging companies' innovation systems to establish merit and if desirable, implementation, in accordance with the realisation component of Mintzberg's model (1994). The model (and this study's adaptation of, Figure 2, p. 15) details the overall innovation input/output sequence. This reinforces von Stamm's (2003) proposition that capabilities harnessing is relative to both strategy and innovation and that effective use of scarce resources is a feature of both exercises. As well, the links with strategy are further evidenced by the converting of concepts to tangible outcome (Kaplan \& Norton, 2000) being similarly achieved using innovation realisation systems.

\section{Innovation Journey Influences}

Objectives Three and Four examine influences on innovation systems. Findings reveal patterns of necessary supports and the inevitable presence of barriers. The lack of stages or intricacy in the basic systems assists to minimise barriers. By confining the basic process to few people with the fewest events necessary expedites the implementing of an innovation and so achieves a rapid outcome. Yet the presence of barriers is not necessarily the issue, as barriers will occur (Bannon \& Grudin, 1990; Council on Competitiveness, 2005; Morrissey, 2000). Rather, results indicate that ensuring ways to control their impact is the key to successfully processing innovations. For instance, effective commitment of scarce resources is supported by feasibility checks, seeking advice, research and carefully evaluating implications.

\section{Triptych Influence Areas}

Reviewing responses under the three areas of Resources, Processes and Values (discussed p. 25) shows little difference between them with barriers relatively well spread across all three. However, the tally of facilitators suggests that Processes and Values are important areas, strongly supportive to realising innovation outcome across the businesses. The area of Resources is marginally less important. However, interviewees' responses varied according to individual interpretation as to the need for a particular positive influence (for instance, the various attitudes to rewarding staff).

Key influences are identified as best use of scarce resources, the need for a champion in resource demanding or complex innovations and a company environment effectively sponsoring innovation activities (negating the need for 
specific training). Rewarding individuals for innovation initiatives is not considered relevant, in fact most likely contrary to successful innovation generation and realisation.

That company values are critical influences to innovation realisation and to overcome barriers is evident in the inclusion of all relevant staff and openness of communication. The innovation systems facilitate collecting feedback and comments to improve innovation outcome, these in turn helping with persuasion and implementation as any concerns potentially hindering adoption have been aired. The inter-relatedness of culture to the innovation journey is a consistent pattern in case study companies. Use of the Resources, Processes and Values triptych to analyse the influences reveals the importance of company culture and operations to the success of innovation outcome (Christensen \& Raynor, 2003).

\section{Innovation to Outcome System Events}

Rogers' Innovation Decision Process as Theoretical Construct

To further understanding of the first objective: identifying case study companies' innovation system components, the results reveal a number of recurring themes. Analysing the research results in conjunction with Rogers' Innovation Decision Process (2003) model provides the framework for consistently considering the gathered data. The five steps of the model are: Knowledge, Persuasion, Decision, Implementation, and Confirmation (details p. 19).

\section{Knowledge.}

This is the rudimentary step of communicating the concept to others and is invariably the first step across the companies, across systems. Knowledge is the critical sharing of an innovation in its nascent state, the trialling the concept with one or more of the following:

- Line manager: this is either the person to whom the individual reports or the line manager responsible for the division that the innovation relates.

- Individual(s) from the employee's peer group, often undertaken to ensure concept feasibility on the most basic level.

- Directly approaching a senior executive: usually occurring if the idea is a time-limited opportunity and the idea generator has thought well through the concept to ensure legitimacy.

- A trusted person (who may or may not be one of the above). 
Even the more sophisticated innovative ideas (including radical concepts) generated at senior level in the organisations receive this feasibility test before progressing further, which often helps to temper the extreme nature of the concepts. Often the inclusion of senior managers at this stage, particularly if resources are required or the innovation has wide impact, can circumvent the need for further efforts if these people are enthusiastic about the innovation from early stages.

As observed by Nonaka and Takeuchi (1995) this socialising of the innovation is paramount. Without opportunities to openly speak about a concept, for a firm to provide an environment that does not ridicule any ideas and provides occasions for expressing these (whether casual chats, team meetings or brainstorming forums) terminates an innovation before a system is initiated. Rogers (2003) stipulates the criticalness of this stage throughout his work on the diffusion of innovation, as does Amabile (1998) in discussing how to kill creativity.

\section{Persuasion.}

Acceptance of the innovation at conceptual stage can include or lead into Rogers' Persuasion step. This is convincing people potentially affected by the innovation of its merits, which may occur whilst introducing the innovation.

Acceptance at Knowledge step results in agreement to progress the innovative concept. To facilitate adopting the innovation, buy-in is achieved through explaining the concept benefits to all necessary employees, most often during meetings. As well, these activities provide a medium for further considering innovation implications; gather opinions, insights and additional feedback. An outcome of meeting(s) may be the re-scoping of the concept and re-presenting. Circular feedback can transpire improving the innovation, corresponding with Rogers' (2003) re-invention of the concept to address concerns.

The use of gentle persuasion discussions (thereby combining Rogers' first two steps) assists the innovation at further stages by reducing the surprise element. The building of persuasion or buy-in reduces outright rejection which may occur through lack of understanding of an innovation's offering. Developing interest in the innovation through informal communication helps to adjust individuals to consider the change as positive. Should this not be the case, attempts that are more definitive are made to achieve this objective. Endeavours may take the form of directly persuading a reluctant individual and affiliation of the concept with hitherto successfully employed innovations to assist acceptance. 


\section{Decision.}

This is choosing the future of the innovation, whether it proceeds or not. In progressing incremental innovation, it is usually collapsed with Persuasion. With more demanding innovations, Persuasion requires the holding of one or more meetings to ensure inclusion of all necessary personnel (especially decision makers). The larger the company or the more resource-demanding an innovation, the more meetings are required, often leading to delays and unintentional barriers. Companies aid the Decision step by reducing the frequency of meetings through ensuring the appropriate people are present from the onset, and including individuals who can decide outcomes in early stages. The objectives of meetings are to give depth of thought to an innovation, its costs and benefits either verbally or formally with documentation. A feedback loop sometimes occurs relating to Rogers' (2003) explanation of this stage as the desire for more information being an element of the decision process. Requiring further information may be necessary to support evaluation of an innovation.

Prioritising innovations as to their value to a company and identifying best resource allocation are key features to evaluating the innovation at this step. As resources often have multiple demands upon them, ranking innovations is critical to then identify best use of limited resources. This juggling of scarce resources demands close examination of innovations whilst not hampering opportunities to grow business.

\section{Implementation.}

Once the decision to adopt an innovation occurs, implementation is often simply a logical extension or minor alternation to current operational activities. This may be as simple as an individual offering an idea, gaining agreement and then changing work practices accordingly. Implementation may require a person to champion the innovation, depending on the innovation's intricacy and implications. The champion, if required, is involved from the start and enthusiastic about the concept supporting it through to realisation. Whether the champion is the idea generator or someone more suitable relates to the type and influence of the innovation. There is no specific level of employee that may be the innovation advocate, however if the innovation has major implications it will most likely be a senior person. 
Some innovations result in the initiating of a project. The case study organisations consider this a separate process with the innovation system terminating on a project commencing. Therefore, once a decision to proceed is made, Implementation takes place as either:

- normal work practices changing, or

- a project being initiated.

That these options result in innovation termination is supported by Van de Ven et al (1999) and Zaltman, Duncan and Holbeck (1973). They propose that such legitimisation translates the innovation into a change activity and the loss of innovation as a separate entity.

\section{Confirmation.}

The last phase is reviewing the innovation, establishing its veracity. The results show that this review step seldom occurs unless the innovation is obviously cumbersome or ineffectual. However, informal revising of innovations occurs as part of continuous improvement philosophy by the organisations. The ongoing scrutiny is likely to reveal an employed innovation that is obviously inefficient, which is then terminated. Conversely, particularly advantageous innovations may be extended for adopting elsewhere in a company.

Reviewing innovation system events from the perspective of Rogers' model shows a consistently linear sequential progression of innovation realisation activities. As new innovations or newcomers to an innovation are introduced, the steps reoccur consecutively to ensure understanding and support adoption. The constant process of introducing the concept requires replicating the Knowledge, Persuasion and to a certain extent Decision steps. However, actual activities may give the appearance as being less sequential as earlier converts work on developing an innovation, refining and re-introducing, repeating the cyclical feedback process, appearing to interfere with the straightforwardness of the process.

\section{System Features.}

The merging of Rogers' (2003) steps is a feature of many of the actual case study systems contradicting the model's explicit five steps. For instance, Persuasion is often part of the first sharing of the concept (Knowledge). Decision is made promptly as a function of successful Persuasion, subsequently immediate Implementation occurs For example, with simplistic incremental innovations there is little differentiation between steps with the events in the entire process taking place 
almost simultaneously with minimal time lags (sometimes only minutes) between steps.

The series of steps comprising Majaro's (1998) (cited in Ahonen (2005)) model's four generic stages of idea generation, screening, feasibility and implementation is also evident in the systems. The stages differ to Rogers' (2003) five steps. However, they can be overlaid on the systems as the first relates to Knowledge, screening and feasibility are part of Decision and the last step relating to Implementation. Both Rogers (2003) and Majaro's (2005) systems provide some generic event sequences for the practical stages as describing intent of each event. Consequently, these reinforce von Stamm's (2003) statement that explanations of stages may differ but overall the processes remain the same.

\section{System Reviews and Success of Current Systems}

The companies in this study do not undertake intentional analyses of their innovation systems. Nor do the companies conduct reviews of the success of implemented innovations other than as occurring as continual improvement revisions of all activities. As such, this limits comprehending the level of success of the systems and innovations. It could be argued as well that lack of knowledge as to what is effective and ineffective hampers developing the systems to better suit company requirements (should this be required). Nevertheless, the systems appear effective in realising innovation outcome with the basic ones functioning well to provide continuous improvements. Similarly, the more serious innovations (platform and radical) are also effectively realising growth for the three companies whilst balancing the inherent risk.

Unquantifiable are the lost innovative ideas, the potential of terminated or parked innovations. This is a common theme across the companies, this loss of intellectual property contained in rejected and parked innovations. There is presently no dedicated company system used by any of the organisations for retention and resurrection of these concepts should their status change. Nor are there facilities for filing information on details why rejected innovations do not proceed or why some innovations fail. Altogether these represent loss of opportunities to develop innovation knowledge and experience that could further innovation activities more effectively. 


\section{Emerging Models}

This study's fifth objective is to provide a framework for managing innovation realisation. This study therefore presents the following models for consideration. The first model presented results from summarising the findings on the overall impact of an innovation on a company. The impact or influence of an innovation dictates the choice of system and the necessary levels of engagement by staff and management. The second model depicts the practical steps of the innovation system that has emerged from the findings. The third model combines the first two to demonstrate how the influence of an innovation impacts on an innovation system, engaging additional levels of involvement as the affect of an innovation increases.

\section{Innovation Sphere of Influence and Implications to System Selection}

The choice of system best used for realising different types of innovation can be simply represented as consisting of three levels. The complexity of the system increases relative to the influence of the innovation (whether a single person or across divisions), together with resource demands and risk to the company. As one or other of these increases so does the need for formalising the process requiring stringent analysis, research and checks on suitability. Figure 17 displays the relationship between these facets.

Sphere 1 Low key innovation, few people directly involved or impacted by innovation. These are generally incremental improvements to current work practises; for example, an improvement in a reporting process. In a company with a continuous improvement philosophy, a growing entrepreneurial or innovation-agile business, this would be a regular, continual occurrence.

Sphere 2 The innovation is more demanding, influencing a larger number of people, has some resource requirements or may require more of a departure from current activities or practices. For example, development of new software that requires all staff to change to in order to improve work functionality. This type of innovation is also usual in an innovatively-agile company. The innovations are slightly more risky but generally perceived as business as usual to achieve strategic ambitions and growth objectives. 


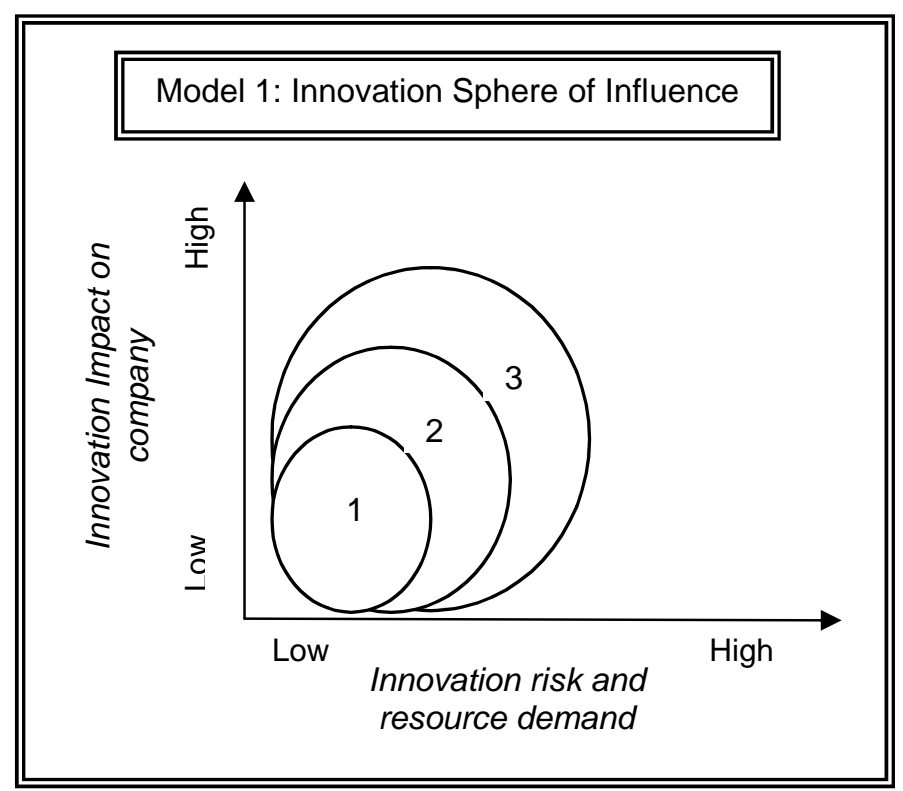

Figure 17 Model 1: Innovation sphere of influence

Sphere 3 Innovations that have a high level of risk, are resource demanding, and have implications to the wider company. These innovations generally demand radical departure from current activities, for instance, opening an office overseas requiring extensive support. This type of innovation and its accompanying system of meetings and checks, extensive research and analysis, is not undertaken lightly. The focus is on understanding implications and securing advantages whilst minimising risk, balancing potential benefits with likely risks.

\section{Emerging Innovation to Outcome Model}

The recurring features of case study companies' ad hoc processes provide a consistency of steps that may be mapped. To this intent, envisaging a potential best practice model can be drawn from process events, as detailed in Model 2 (Figure 18, p. 119). Separate steps lead into the next, or the steps can run concurrently if required. The system ceases at any time through either implementing or terminating the innovative concept. The tailoring of individual generic features is possible to suit a company's specific requirements.

\section{Model 2 Events.}

As demonstrated by the research, socialising the innovation through casual communication is the primary step generating Knowledge of the concept. As indicated by Rogers (2003), this can lead to acceptance (Persuasion), and Decision 
to Implement. Further stages are employed if the innovation's implications indicate the necessity of additional safeguards. These added stages relating to the Persuasion and Decision steps and include the gathering of information on an innovation's implications. One or more meetings may be required to gather such advice and persuade individuals as to merits with cyclical feedback improving and refining the innovation. These three steps of Knowledge, Persuasion and Decision are repeated with each new entrant (whether individual, team or department) at each stage.

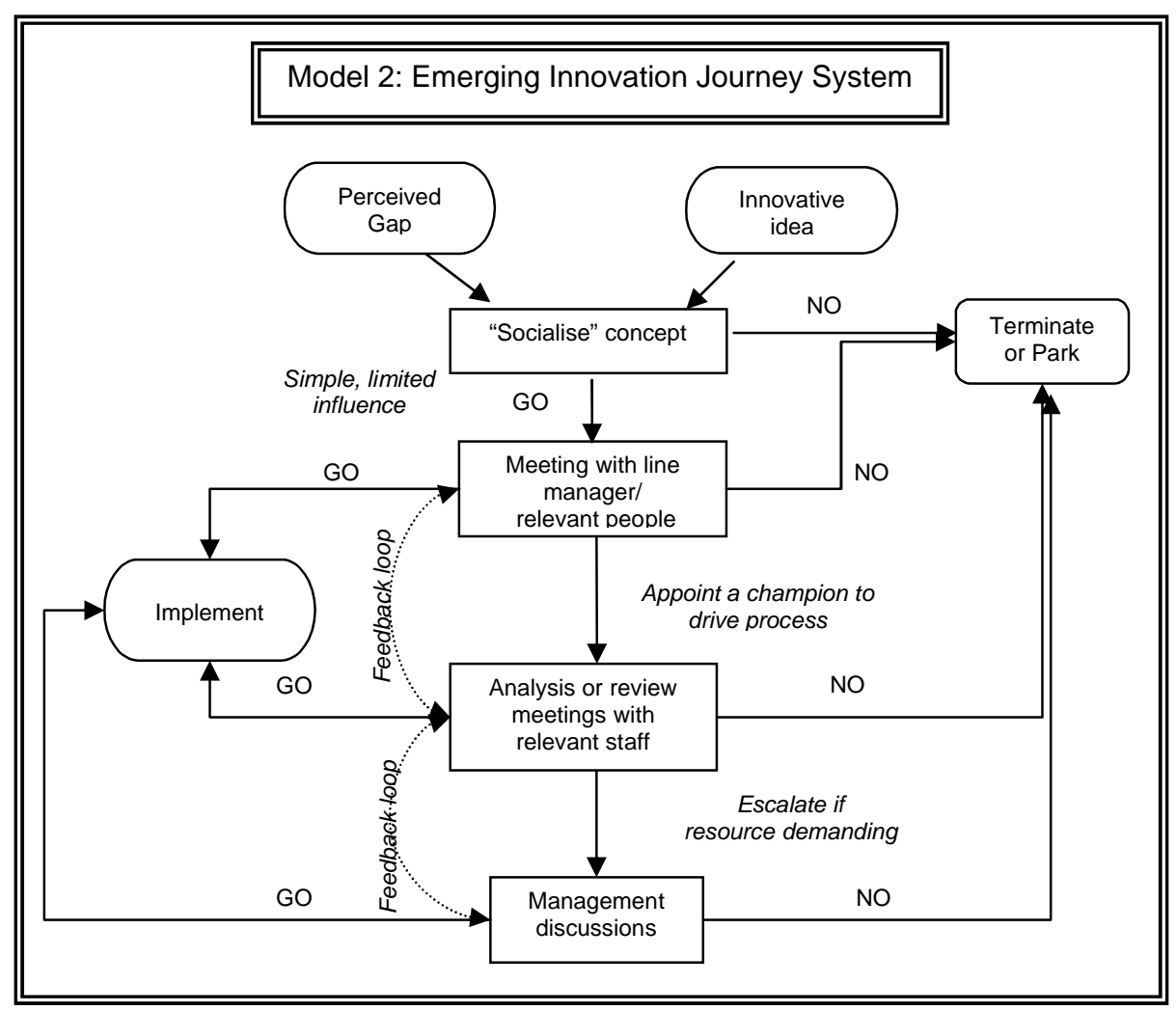

Figure 18: Model 2: Emerging innovation journey system

The addition of a champion may be apparent at this time to support the more complex or demanding innovation. The champion may build impetus, continuing to persuade and recruit advocates for the innovation. If persuasion of senior executives is required, the champion may also need to be a senior executive in order to convince others at high level. The system may escalate to a meeting of executives (and board) to review the innovation, its implications and decide resource use. Deciding whether to implement an innovation relates to these implications which are individual to a company. 


\section{Model 3: Combined Model}

Finally, the two figures can be combined (Figure 19) demonstrating the increase in system complexity as the sphere of influence grows. Model 3 visually represents the intersection between realising the concept and the relevant events a company may require to unlock the value of an innovation. For example, an innovation that has Sphere 2 influence requires additional discussions and advice steps in the system.

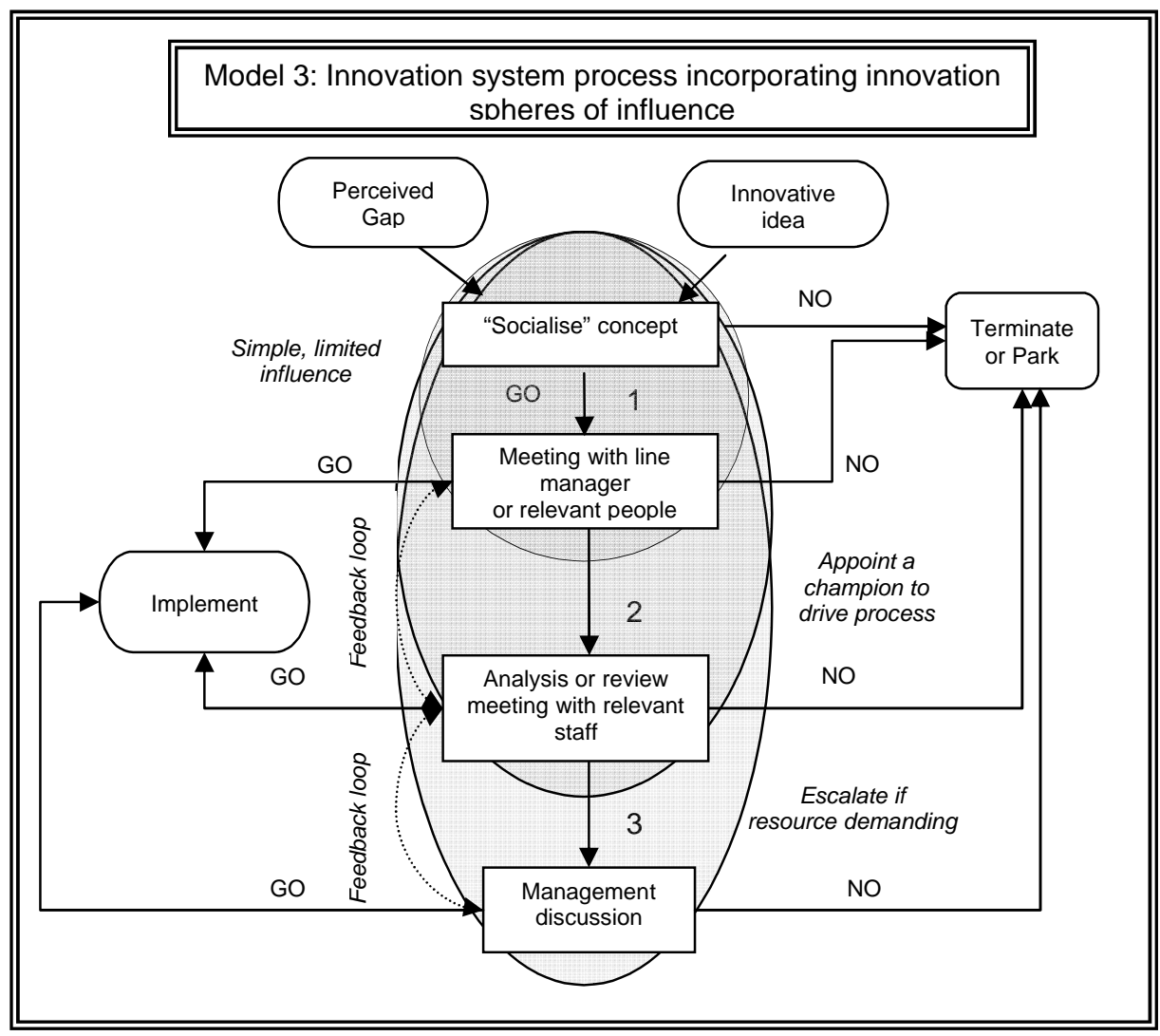

Figure 19: Model 3: Emerging system with innovation spheres of influence

This chapter analysed the results of the study considering both the empirical research and the literature review. The chapter presents key findings in order to improve understanding of the internal innovation journey with discussion of the results culminating in conceptual models for guiding effective innovation systems. The next section presents the conclusions reached in the context of this study. 


\section{Chapter 6 \\ CONCLUSIONS}

\section{Chapter Outline}

The purpose of this study was to ascertain the practical events in managing the internal innovation journey from inception to outcome. As well, to expand recognition of practices that supports the realisation of innovation value. The overall intention is to develop a map of activities and understanding of activities that lead to best practice. This final chapter presents the conclusions of this study. As well, the limitations of the study are summarised at the end of this chapter, together with recommendations for further research.

The principal finding of this study is that the conceptually amorphous nature of the innovation journey expounded by scholars is not evident in actual internal innovation systems employed by companies. The innovation systems practised are overall a straightforward series of events with risk-propensity of an innovation increasing the number of steps. These supplementary steps are necessary to mitigate risk and maximise the effectiveness in realising innovation value. However, the simpler the innovation process the quicker the outcome in order to realise companies' objectives for continuous improvement. The clear functional steps and the actions taken to address implications lend credence to mapping system events (Models 2 p. 119 and 3 p. 120). Variations within and among systems relate to the characteristics of the companies themselves, the industry, clients and business model dictating nuances in the innovation systems.

The events comprising innovation systems are not elaborate and often merge, combining sequential steps simultaneously if required. The processes are all dependent on conveying the innovative concept with confidence to fellow employees, the critical first step to encouraging and facilitating innovation. Implementation often occurs simply as an adjustment to current business or operational activities. With more radical innovations - which usually require a departure from current activities - implementation is the instigating of a project.

Management of the innovation system does not occur as a separate exercise. The innovation journey appears to be interdependent with a company's overall environment. Effective innovation systems in the three case study companies are underpinned by an innovation-orientated culture with the systems not performing in 
isolation. The systems are sub-sets of operational activities and ethos, integral functions of management, staff attitude and work ethics. The innovation environment contains a number of key operational features to facilitate innovation success. The features are primarily a company philosophy based on an open environment together with receptivity to new ideas and change. These features provide a climate supportive of innovation agility pivoting on the crucial aspect of open, free-flowing communication between all levels of employees.

Yet innovation-agile companies face obstacles to unlocking innovation value. The findings suggest that the presence of barriers need not hinder innovation realisation if organisational awareness leads to providing methods for these to be overcome. The impact of barriers appears to be further limited through ensuring dominance of positive innovation influences. Hence, innovation successful organisations have numerous facilitators supporting innovation realisation. Innovation-outcome supports include cultural attitude to innovation, ready access to decision makers, lack of bureaucracy and rigid structures. Case study companies improve innovation realisation and adoption through ensuring these supports.

Innovation automatically disrupts equilibrium by changing current practices. Whether on a minor or major scale, innovation introduces a level of chaos conflicting with the necessary order of a business. That the case study companies are experiencing problems brought about by growth due to the success of their innovation activities is an example of this chaos. The need to balance stability and daily business with continuing innovative activities - which brings disequilibrium - is leading the companies to seek methods to overcome this conundrum. The companies are also aware of the necessary focus of staff on day-to-day activities in the growing organisation limiting opportunities, and interest, in innovation generation.

These problems relate to Griener's (1998) five stages of growth model. The model depicts the evolution of an organisation and the revolutions it experiences as it transits to the next operating level. As well, the model provides indicators of the factors to be aware of at each stage. As well, Lewin's (1951) three phase change process unfreeze-change-refreeze for managing change. Unfreeze relating to the altering of established business practices, then change through introducing new practices and then refreeze to provide stability while new operating methods are embedded. Both these models and similar works may provide assistance to 
organisations facing such challenges by providing techniques to address concerns and greater understanding as to the situation being experienced.

This study develops theoretical understanding and provides practical direction for companies desiring to better understand managing innovation to realise value. It attempts to address an apparent gap in the literature on the practical composition of internal innovation journey with findings growing the knowledge of what constitutes functional steps in unlocking innovation value, the necessary culture and practices to support effective realisation. Results direct the development of an emerging best practice model which further studies can elaborate and define, or refute. The intention is to provide a foundation of events, features and influences relating to the innovation-to-outcome journey which further research and comprehension may then enhance.

\section{Limitations}

The present research project has certain limitations that need to be considered in context of the entire study. However, some of these self-same limitations indicate opportunities for further research. Firstly, the empirical analysis examined three companies' innovation systems in depth and innovation activities in breadth. These three companies were purposely selected as having innovation-to-outcome systems to study hence, were not randomly chosen which potentially limits general applicability. Additionally, while multiple case studies do provide a greater ability for generalisation than a single case may otherwise, nevertheless, three is a minor number. A larger sample would provide more extensive data, therefore greater generalisation.

Selection of one market and the Information Communication Technology (ICT) industry specifically, creates limitations. A relatively new industry, the ICT market is known for its innovation bias; this industry feature was used as part of the selection criteria. However, it could be argued for future research on this topic that different industries, particularly well established ones, would reveal systems and methods different to those existing in the dynamism of the ICT market.

Another limitation is introduced through the interviewing of only senior staff members. The management sample provided data that demonstrated senior employees' perspectives of the innovation systems. Interviewing junior employees would provide a more extensive range of perceptions, system interpretations and 
variations thereof. Similarly, there are concerns as to the social desirability bias resulting from respondents wishing to present their company as innovative. In the same vein, the interviewing of the employees recommended by the senior person may have lead to bias, the senior person selecting people that would offer agreeable views. As well, the use of interviews inherently has limitations - as does any type of primary data collection method - and needed managing accordingly.

Use of Rogers' (2003) model may have caused limitations when qualifying and interpreting the case study companies' systems. Throughout the study it has been argued that Rogers' provides a sound basis for analysis, the type of model and its event sequence both support and define explanation of innovation systems. The providing of a theoretical construct to underpin the study is necessary in such research. However, the use of such definitive parameters as provided by Rogers' model can cause bias in analysis. Similarly, the framework of Rogers' model dictates a linear progression that may not have been as evident otherwise.

Another limitation of this research is the assumption that systems employed by the companies are effective in producing results. The judging of success is dependent on interviewees' opinions, companies' achievements and information held in the public arena. The measures of success are based on, internally, achieving innovation objectives, and externally, indicators such as evidential growth and market share.

Focusing analysis on the innovation system isolated it from possible influences outside the scope of this research. For instance, investigating what constituted implications considered during the decision process, the use of decision process methods, project management principles and other facets impacting on innovation progress and outcome. Finally, whether an innovation system can exist outside of an innovation-orientated culture is beyond the scope of this study. It may be an interesting area to research.

\section{Suggestions for Further Research}

As indicated by the suggestions above there are additional areas that require further study:

Conducting research on companies that have successfully transitioned from an entrepreneurial business to a mid-sized company whilst retaining the attributes of an innovative culture. Such research helps to identify 
and develop understanding of the features and practices necessary to support ongoing innovation activities.

- Modelling the innovation systems of other companies, and businesses in other industries. Analysing this information and then comparing it to the results of this study would provide a more robust innovation system model.

- Investigating methods for capturing parked innovations and the process for resurrecting these suspended innovations. Better management of such innovations could help to realise their intellectual property value and build stronger innovation effectiveness.

- Research could also be undertaken on the techniques to address barriers to innovation realisation. This research could simultaneously investigate methods of promoting enablers providing better understanding of how to manage the influences on unlocking innovation.

That further research is required suggests that a systematic approach to innovation management may provide improved understanding, therefore improved innovation realisation success.

\section{In Summary}

Companies with a desire to pursue innovation as a way to augment their business may achieve this through encouraging employees to propose any ideas for consideration, from subtle changes through to major alterations to standard business practice. The encouraging of innovative ideas to be complemented by an organisational climate and structure that supports innovation and realising the value of these through employing an innovation system such as described in this study.

This study has developed knowledge of the practical innovation journey, increasing awareness through analysis of actual working systems. Better understanding of events assists businesses to more effectively realise the value and opportunities innovative ideas represent. This then leading to more effectively manage the innovation journey and success in unlocking the value contained within innovations. 


\section{REFERENCES}

Ahonen, M. (2005). Designing Information Systems for Creative Problem Solving and Learning (Research report). Tampere: University of Tampere, Finland.

Allen, K. R. (2003). Bringing New Technology to Market. USA: Prentice Hall.

Amabile, T. M. (1998). How to kill creativity. Harvard Business Review, 77-87.

Annacchino, P. E. (2003). New Product Development: from initial idea to product management. USA: Elsevier.

Bannon, L. J., \& Grudin, J. (1990). Organizational Barriers to Innovation in IT Development and Use. Paper presented at the 13th Iris Conference, Turku, Finland.

Batiz-Lazo, B., \& Woldesenbet, K. (2006a). The Dynamics of Product and Process Innovation in UK Banking. International Journal of Financial Services Management, UK.

Batiz-Lazo, B., \& Woldesenbet, K. (2006b). The Dynamics of Product and Process Innovation in UK Banking. International Journal of Financial Services Management.

Batonda, G., \& Perry, C. (2001). Approaches to relationship development processes in inter-firm networks. European Journal of Marketing, Vol 37(10), P.14571484.

Bean, R., \& Radford, R. (2002). The business of innovation. USA: Amacom Books.

Birkinshaw, J., Hamal, G., \& Mol, M. (2005). Management Innovation : working paper. London: London Business School.

Boston Consulting Group. (2005). Innovation 2005 (Survey).

Bouchard, V. (2001). Exploring corporate entrepreneurship: A corporate strategy perspective. Paper presented at the 17th EGOS Conference, Lyon.

Bouma, G. (2000). The Research Process (Fourth ed.). Australia: Oxford University Press.

Carson, D., Gilmore, A., Perry, C., \& Gronhaug, K. (2001). Qualitative Marketing Research. Lndon: Sage Publishing.

Cavana, R. Y., Delahaye, B. L., \& Sekaran, U. (2000). Applied Business Research: Qualitative and Quantitative Methods: John Wiley \& Sons Australia, Ltd.

Chase, R. B. (1978). Where Does the Customer Fit in a Service Organisation. Harvard Business Review, 56 (6), 137-142.

Chase, R. B., \& Hayes, R. H. (1991). Beefing up Operations in Service. Sloan Management Review, Fall, Vol 33(No. 1).

Cheng, Y., \& Van de Ven, A. (1996). Learning the Innovation Journey: Order out of Chaos? Organization Science, Vol. 7(Issue 6), p.593-614. 
Chesbrough, H., \& Rosenbloom, R. (2002). The role of the business model in capturing value from innovation: evidence from Xerox Corporation's technology spin-off companies. Industrial and Corporate Change, 11(3), 529-555.

Christensen, C. M. (1997). The Innovator's Dilemma. USA: Harvard Business School Press.

Christensen, C. M., Anthony, S. D., \& Roth, E. A. (2004). Seeing What's Next. USA: Harvard Business School Press.

Christensen, C. M., \& Raynor, M. E. (2003). The Innovator's Solution. USA: Harvard Business School Press.

Christensen, K. S. (2005). Enabling intrapreneurship: the case of a knowledgeintensive industrial company. European Journal of Innovation Management, 8(3), 305-322.

Collis, J., \& Hussey, R. (2003). Business Research (Second ed.). New York: Palgrave MacMillan.

Commission of the European Communities. (2003). Innovation policy: updating the Union's approach in the context of the Lisbon strategy. Brussels: Commission to the Council, the European Parliament, the European economic and social committee and the committee of the regions.

Conseil de la recherche forestiere du Quebec. (2004). Innovation through the Mastery of Knowledge. Quebec.

Cooper, D. R., \& Edgett, S. J. (2001). Portfolio Management for New Products. Canada: Product Development Institute.

Cooper, J. R. (1998). A multidimensional approach to the adoption of innovation. management Decision, 36( 8), 493-502.

Cooper, R. G. (2001). Winning at new products (Third ed.). USA: Basic Books.

Cooper, R. G. (2005a). Product Leadership. USA: Basic Books.

Cooper, R. G. (2005b). Winning at New Products: Pathways to Profitable Innovation: Product Development Institute.

Council on Competitiveness. (2005). 2005 National Innovation Survey. USA: Council on Competitiveness.

Davenport, T. (2006). The Different Domains of Innovation. Babson Insight.

Davenport, T., Prusak, L., \& Wilson, H. (2003). Who's bringing you hot ideas? Harvard Business Review(February), p. 58-64.

Day, J. D., Mang, P. Y., Richter, A., \& Roberts, J. (2001). The innovative organization. McKinsey Quarterly( 2), p.21-31.

de Vaus, D. (2001). Research Design in Social Research. UK: Sage Publications.

Drucker, P. (1985a). The discipline of Innovation. Harvard Business School(MayJune), p.67-72. 
Drucker, P. F. (1985b). Innovation and Entrepreneurship. UK: Pan Business Management.

Eisenhardt, K. M. (1989). Building Theories from Case Study Research. Academy of Management Review, 14(4), p. 532-550.

Fielden, K., \& Malcolm, P. (2006, June 12 - 14). Organisational Pathways: Creativity to Productivity. Paper presented at the European Conference on Information Systems, Sweden.

Frederick, H. (2006). Entrepreneurship: Theory, Process and Practise (Asia Pacific Edition). New Zealand: Thomson Learning.

Garvin, D., \& Levesque, L. (2004). Emerging Business Opportunities at IBM. Harvard Business School(9-304-075).

Govindarajan, V., \& Trimble, C. (2005). Building breakthrough businesses within established organisations. Harvard Business Review, p. 60-68.

Greiner, L. E. (1998). Evolution and Revolution as Organizations Grow. Harvard Business Review, 55-67.

Hamel, G. (2000). Waking up IBM. Harvard Business Review, 137-146.

Hamel, G. (2006). The Why, What and How of Management Innovation. Harvard Business Review, 72-84.

Hamel, G., \& Getz, G. (2004). Funding Growth in an Age of Austerity. Harvard Business Review, 76-84.

Hamel, G., \& Prahalad, C. K. (1994). Competing for the Future. USA: Harvard Business School Press.

Hamel, J., Dufour, S., \& Fortin, D. (1993). Case Study Methods. USA: Sage Publications.

Handy, C. (2002). Elephants and Fleas: Is your organization prepared for change? Leader to Leader, 24.

Harvard Business Review. (2002). The Innovative Enterprise. Harvard Business Review(August), 39 - 49.

Hesselbein, F., Goldsmith, M., \& Sommerville, I. E. (2002). Leading for innovation. New York, USA: Jossey-Bass.

Hoffman, R., \& Hegarty, W. (1993). Top Management Influence on Innovations: Effects of Executive Characteristics and Social Culture. Journal of Management, Vol. 19( No.3), p. 549-574.

IBM Global Business Services. (2006). The Global CEO Study 2006: Expanding the Innovation Horizon.

Jankowicz, A. (1992). Business Research Projects for Students. London: Chapman and Hall. 
Jauhari, V. (2001, March 11 - 14). Corporate Entrepreneurship : A Framework for Competitiveness. Paper presented at the Tenth International Conference on Management of Technology, Lausanne, Switzerland.

Jones, O. (2002a). Innovation and Organizational Change: Mobilising Social Capital through Corporate Entrepreneurship. Manchester: Manchester Metropolitan University Business School.

Jones, T. (2002b). Innovating at the Edge. Oxford: Butterworth-Heinemann.

Kanter, R. M. (1982). The Middle Manager as Innovator. Harvard Business Review, 95-105.

Kanter, R. M. (1985). The Change Masters. New York: Simon and Schuster.

Kanter, R. M. (1989). When giants learn to dance. Sydney: Allen \& Unwin.

Kanter, R. M. (2002). Creating the Culture for Innovation. In M. G. F Hesselbein, I Somerville (Ed.), Leading for Innovation (pp. 79-85). San Francisco: JosseyBass.

Kaplan, R. S., \& Norton, D. P. (1992). The Balanced Scorecard - Measures that drive performance. Harvard Business Review, 70.

Kaplan, R. S., \& Norton, D. P. (2000). Having trouble with your strategy? Then map it. Harvard Business Review(September-October), p.167-176.

Katz, R. (2004). The human side of managing technological innovation: A collection of readings (2nd ed.). New York: Oxford University Press.

Keats, D. M. (2000). Interviewing. Australia: UNSW Press.

Khurana, A., \& Rosenthal, S. (2002). Integrating the Fuzzy Front End of New Product Development. In M. S. M. Review (Ed.), Innovation: Driving Product, Process and Market Change. San Francisco: Jossey-Bass.

Knight, R. (1987). Corporate Innovation and Entrepreneurship: A Canadian Study. The Journal of Product Innovation Management(4), 284-297.

Kohlbacher, F. (2005). The use of qualitative content analysis in case study research. Forum: Qualitative Social Research (On line Journal), 7(1).

Kotter, J. P. (1996). Leading Change. USA: Harvard Business School Press.

Kuratko, D. F., \& Hornsby, J. S. (2001, 7 - 10 February). Corporate Entrepreneurship and Middle Managers: A model for corporate entrepreneurial behavior. Paper presented at the 2001 USASBE/SBIDA Annual National Conference, Orlando, Florida.

Lewin, K. (1951). Field Theory in Social Science. New York: Harper \& Row.

Machiavelli, N. (1950). The Prince and The Discourses. New York: The Modern Library, Random House, Inc. 
Maidique, M. A., \& Hayes, R. H. (1998). Executive Leadership and the Management of Innovation and Change. In M. L. T. W. L. Moore (Ed.), Readings in the Management of Innovation (pp. 689-704). USA: HarperBusiness.

Majaro, S. (1998). The Creative Gap - Managing Ideas for Profit. United Kingdom: Longman.

Marshall, C., \& Rossman, G. (1999). Designing Qualitative Research (3rd Edition ed.). USA: Sage Publications.

McAdam, R. (2005). A multi-level theory of innovation implementation. European Journal of Innovation Management, 8(3), 373-388.

McAdam, R., Stevenson, P., \& Armstrong, G. (2000). Innovative change management in SMEs: beyond continuous improvement. Logistics Information Management, Vol. 13(Issue 3), p. 138-149.

McFadzean, E., O'Loughlin, A., \& Shaw, E. (2005). Corporate entrepreneurship and innovation part 1: the missing link. European Journal of Innovation Management, 8(3), 350-372.

Mclntyre, S. H. (2001). Obstacles to Corporate Innovation. Business Horizons, 2328.

McLaughlin, P., Baessant, J., \& Smart, P. (2005). Developing an Organizational Culture that Facilitates Radical Innovation in a Mature Small to Medium Sized Company: Emergent Findings. Milton Keynes: The Cranfield School of Management.

Miles, M. B., \& Huberman, A. M. (1994). Qualitative Data Analysis (Second ed.): Sage Publications.

Mintzberg, H. (1994). The rise and fall of strategic planning. USA: Prentice Hall.

Morrissey, C. A. (2000). Managing innovation through corporate venturing. California: The Graziadio School of Business and Management.

Morse, J. M., \& Richards, L. (2002). Read Me First: a User's Guide to Qualitative Methods: Sage Publications.

Nemeth, C. J. (1997). Managing Innovation, When Less is More. California Management Review, 40(1).

New Zealand Trade and Enterprise. (2006). Information and Communications Technology (ICT) - Delivering superior value. Retrieved 03/07, 2006, from http://www.nzte.govt.nz/section/11757.aspx

Nonaka, I., \& Takeuchi, H. (1995). The Knowledge-Creating Company. New York: Oxford University Press.

Nutley, S., Davies, H., \& Walter, I. (2002). Learning from the Diffusion of Innovations. St Andrews, UK: Research Unit for Research Utilisation, University of St Andrews. 
Papadakis, V., \& Bourantas, D. (1998). The CEO as corporate champion of technological innovation: an empirical investigation. Technology Analysis and Strategic Management, 10,1, 89-109.

Patton, M. Q. (1980). Qualitative Evaluation and Research Methods (Second ed.). USA: Sage Publications.

Pavitt, K. (1991). What makes basic research economically useful. Research Policy, 20.

Pavitt, K. (2003). The Process of Innovation (Working paper). Sussex, UK: The Freeman Centre, University of Sussex.

Peel, S. (2003). The costs and benefits of alternative employment structures. Unpublished PhD, University of Auckland, Auckland, New Zealand.

Peters, T., \& Waterman, R. (1982). In Search of Excellence. New York: Harper and Row.

Phillips, J., \& Hering, D. (2005). Innovate on purpose. USA: NetCentrics Corporation.

Pinchot, G. (1985). Intrapreneuring. New York: Harper \& Row.

Pinchot, G. (1987). Innovation Through Intrapreneuring. Research Management, $X X X(2)$.

Pinchot, G., \& Pellman, R. (1999). Intrapreneuring In Action: A handbook for business innovation. San Francisco: Berrett-Koehler Publishers.

Pirich, A., Knuckey, S., \& Campbell, J. (2001). An interface between entrepreneurship and innovation - New Zealand SMEs perspective. Paper presented at the Druid Nelson and Winter Conference 2001, Aalbory University, Denmark.

Roberts, E. B., \& Fusfeld, A. R. (2004). Critical Function: Need Roles in the Innovation Process. In R. Katz (Ed.), The Human Side of Managing Technological Innovation: A collection of readings (pp. 246-260). NY: Oxford University Press.

Rogers, E. (2003). Diffusion of Innovation (5th ed.). USA: Free Press.

Rogers, E., \& Singhal, A. (1996). Diffusion of Innovation. Norwodd, NJ, USA: Lawrence Erlbaum Associates.

Rudestam, K., \& Newton, R. (2001). Surviving Your Dissertation (Second Edition ed.). USA: Sage Publications.

Sathe, V. (2003). Corporate entrepreneurship: top managers and new business creation. UK: Cambridge Press.

Savery, C. A. (2005). Innovators or Laggards: surveying diffusion of innovations by public relations practitioners. Unpublished Master's, University of Akron, Akron, Ohio, USA. 
Schroeder, R., Van de Ven, A., Scudder, G., \& Polley, D. (1986). Managing Innovation and Change Processes: Findings from the Minnesota Innovation Research Program. Agribusiness, Vol 2(4), p. 501-523.

Schumpeter, J. (1942). Capitalism, socialism, and democracy. New York: Harper \& Row.

Senge, P. (1992). The Fifth Discipline. Sydney: Random House.

Sharma, A. (1999). Central dilemmas of managing innovation in large firms. California Management Review, 41(3), 146-164.

Sheppard, B., \& Canning, M. (2006). Innovation Culture. Leadership Excellence, 23, $1,18$.

Somekh, B., \& Lewin, C. (2005). Research Methods in the Social Sciences: Sage Publications.

Soy, S. (2006). The Case Study as a Research Method

Srivastava, M., \& Gnyawali. (2006). Overcoming Innovation Barriers: An Ego Network Perspective. Blacksburg, VA: Virginia Polytechnic Institute and State University.

Statistics New Zealand. (2004). Innovation Survey 2003 (Research in conjunction with MoRST). New Zealand: Ministry of Research, Science and Technology (MoRST).

Tarrant, D. (2005a). Big, Bold Goals - the Innovation Imperative. Australian Graduate School and Management( 2).

Tarrant, D. (2005b). New Centre to deliver innovation to the real world. Australian Graduate School and Management( 03).

Tellis, W. (1997). Introduction to Case Study. The Qualitative Report, Vol 3,(2).

Thomas, R. M. (2003). Blending Qualitative and Quantitative Research Methods. California: Sage Publications.

Thornberry, N. (2001). Corporate Entrepreneurship: Antidote or Oxymoron? European Management Journal, 19(, 5), p. 526-533.

Thornberry, N. (2006). Lead like an Entrepreneur. New York: McGraw Hill.

Tidd, J., Bessant, j., \& Pavitt, K. (1998). Managing Innovation. England: John Wiley \& Sons.

Tidd, J., \& Hull, F. (2003). Service innovation. London: Imperial College Press.

Toftoy, C., \& Chatterjee, J. (2004, June). The Intrepreneurial Revolution: Now is the time for action. Paper presented at the International Council for Small Business 2005 Conference, USA.

Trochim, W. M. K. (2006). Nonprobability Sampling. Retrieved 21 February, 2007, from www.socialresearchmethods.net/kb/sampnon.php 
Umit, H. (2005). Case Studies: Methodologie de la Recherche en Sciences de Gestion. Belgique: Universite Catholique de Louvain Institut d'Administration ed de Gestion.

Van de Ven, A. (1986). Central Problems in the Management of Innovation. Management Science, Vol.32(No. 5), p. 590-607.

Van de Ven, A. (1993). Managing the Process of Organizational Innovation. In G. Huber \& W. Glick (Eds.), Organizational Change and Redesign: Ideas and Insights for Improving Performance (pp. p.269-294). New York: Oxford Press.

Van de Ven, A., \& Engleman, R. (2004). Central Problems in Managing Corporate Innovation and Entrepreneurship. Advances in Entrepreneurship, Firm Emergence and Growth, Vol. 7, p.47-72.

Van de Ven, A., Polley, D., Garud, R., \& Venkataraman, S. (1999). The Innovation Journey. New York: Oxford University Press.

Van de Ven, A., \& Rogers, E. (1988). Innovations and Organizations: Critical Perspective (Discussion Paper \#96). Minnesota: Strategic Management Research Center.

VanGundy, A. B. (2005). The care and framing of strategic innovation challenges. Oklahoma: University of Oklahoma.

von Stamm, B. (2003). Managing Innovation, Design \& Creativity. England: John Wiley \& Sons.

Weitzel, D., \& Hallahan, K. (2003). Organizational adoption of an intranet-based performance reporting system: A test of Rogers' model of innovation. Colorado State University, Colorado.

Weller, S., Green, S., \& Fernie, S. (2004). Learning Across Business Sectors: Facets of Innovation in Aerospace and Construction. Paper presented at the Project Procurement for Infrastructure Construction, India.

Wengraf, T. (2001). Qualitative Research Interviewing. UK: Sage Publications.

Williams, A. (1999). Creativity, Invention \& Innovation. Australia: Allen \& Unwin.

Wilson, M. E. (2005). Case in Point. Auckland: GSE Publications.

Woodside, A., \& Biemans, W. (2005). Modeling innovation, manufacturing, diffusion and adoption/rejection processes. Journal of Business and Industrial Marketing, 20/7, p, 380-394.

Yin, R. K. (2003a). Applications of Case Study Research (Second ed.): Sage Publications.

Yin, R. K. (2003b). Case Study Research: Design and Methods (Vol. Five): Sage Publications.

Yin, R. K. (2004). The Case Study Anthology: Sage Publications.

Zaltman, G. (1973). Processes and Phenomena of Social Change. New York: John Wiley \& Sons. 
Zaltman, G., Duncan, R., \& Holbeck, J. (1973). Innovations and Organizations. USA: John Wiley \& Sons.

Zien, K. A., \& Buckler, S. A. (2004). Dreams to Market: Crafting a Culture of Innovation. In R. Katz (Ed.), The Human Side of Managing Technological Innovation (pp. 478- 493). NY: Oxford University Press. 
APPENDICES 
APPENDIX A

Information Sheet for Participants 


\section{UUnitec}

\section{Information for Participants}

From inception to outcome: Managing the internal pathway of innovation

My name is Fern Evitt; I am a postgraduate student at Unitec studying for a MBIE: Master of Business Innovation and Entrepreneurship. Part of the degree programme involves a research project or thesis.

\section{What I am researching}

My thesis proposes to identify and analyse the internal innovation process implemented by a company. In other words, how a company transforms an innovation through the organisation into an outcome or result of value to the firm. Businesses are being told that innovation is critical to future successes. Yet how a company can best take the innovative ideas generated and turn them into realisable outcomes - whether a better system of doing things, improved services, a new product - is not clear.

The project aims to understand what stages or steps occur in a company when an employee comes up with an innovative concept. A company ideally has a systematic process by which it transforms the idea into an outcome of some value to the business. What does this look like? What perhaps are the stages? Does a business plan get written? Does the originator stay involved? What works or doesn't work? The research project will study companies who have already developed a process, whether ad hoc or more formalised system.

\section{What it will mean for you}

I wish to interview you to ask about:

- The internal innovation process used by your company, and its features.

- Mapping or flow charting the process and finding out about the different stages and any problems.

It is envisaged that this initial interview will take approximately one hour. Some further clarification, via email or telephone (which ever most convenient to you) may be necessary during compiling results to ensure accuracy and that I have understood you correctly. Notes will be taken during the interview, which will be recorded (if acceptable to you) and later transcribed. All features that could identify you will be removed and the recording erased, once the transcription is completed.

You are free to withdraw from this project within two weeks of the interview being conducted, or terminate the interview for whatever reason at any stage.

Research findings will be anonymous with identities of any companies being disguised. Individual participants' names will also be disguised or not referred to in the report. Confidential or commercially sensitive information will not be sought nor collected. 
By taking part in this research you will be assisting the researcher to identify methods and systems employed by companies in the transformation process. This may lead to concepts or precepts of best practice. I am considering undertaking a $\mathrm{PhD}$ in the future; consequently, some of the core information gathered during this project may be re-used for this purpose.

\section{Objective of the Research}

For those involved and business in general, findings of the research may assist in considering the efficacy of currently employed innovation to outcome processes. Through analysis and correlation of findings, a model of best practise may emerge which may be tested by further research.

\section{Confidentiality and Anonymity}

Your name and any information that may identify you will be kept completely confidential. All information collected from you will be stored on a password protected file with the only access to your information is yourself, the researcher and my two supervisors. The information you supply will have no identifiers ensuring your anonymity in the study.

\section{Consent}

If you agree to participate, you will be given a consent form to sign. This does not stop you from changing your mind if you wish to withdraw from the project.

Please contact me if you need more information about any aspects of this research project. At any time if you have any concerns about the project you can contact my supervisor: Dr Simon Peel, Postgraduate Programme Director, Unitec tel. 8154321 extn 8650 .

\section{Thank you for your consideration.}

Fern Evitt

Project researcher

Telephone: 021927093

fernevitt@hotmail.com

This study has been approved by the Unitec Research Ethics Committee from 23 August 2005 to 30 June 2007. If you have any complaints or reservations about the 
ethical conduct of this research, you may contact the Committee through the UREC Secretariat (Ph: 098154321 ext.7254). Any issues you raise will be treated in confidence and investigated fully, and you will be informed of the outcome. 


\section{APPENDIX B}

Interview Consent Form 
From inception to outcome: Managing the internal pathway of innovation

\section{This consent form is to facilitate the gathering of information for a Master of Business Innovation and Entrepreneurship (MBIE) thesis. The research is looking at the internal system or process by which a company transforms an innovation into an outcome or result of value to the business.}

I have had the research project explained to me and I have read and understand the information sheet given to me. I understand that I don't have to be part of this if I don't want to and I may withdraw at any time within two weeks of the interview being conducted.

I understand that everything I say is confidential and none of the information I give will identify me. The only persons who will know what I have said will be the researcher and her supervisors (2 people). I also understand that all the information that I give will be stored securely on a computer for a period of 5 years. I understand that my discussion with the researcher will be taped and transcribed. I understand that I can terminate the interview at any stage.

I understand that I can see the finished research document. I also am aware that some of the core information gathered during this project may be re-used in pursuit of a future PhD by F Evitt. I am aware that I may contact the Researcher, Fern Evitt on 021927093 or her Research Supervisor, Dr Simon Peel at Unitec, (09) 815-4321 ext. 8650, if I have any queries about the project.

I have had time to consider everything and I give my consent to be a part of this research project.

This study has been approved by the Unitec Research Ethics Committee from 23 August 2005 to 30 June 2007. If you have any complaints or reservations about the ethical conduct of this research, you may contact the Committee through the UREC Secretariat (Ph: 09 815-4321 ext 7254). Any issues you raise will be treated in confidence and investigated fully, and you will be informed of the outcome.

Participant Name:

Signature: Date: 



\section{APPENDIX C}

Interview Guideline 


\section{Interview Guideline}

(Specific notes for interviewer in italics)

Check if any questions and that the interviewee is again happy to give consent to be interviewed and the interview recorded.

Establish definition and relevance of innovation as understood by interviewee

- What does the term innovation mean to you?

- $\quad$ Do you see it as important in business today?

Part One

Mapping the internal process employed by the company

1 (Introduce the concept) eg. "Someone comes up with what they think is a great idea, of benefit to the company. What then happens from your experience?"

1.a Would it be possible to walk through the various steps for me?

1.b What are the features of each step?

1.c When is it time to move on to the next step?

Identify if different versions exist for different types of innovations

1.d "Does this process differ if the innovation is a tangible one (eg. products) or intangible (eg. services, process improvements)?"

Or if the innovation is radical, or a less demanding incremental innovation?

Part Two

Barriers and facilitators to the system

Produce list of barriers and enablers

2.1 "In your experience, have you noticed these barriers to innovation outcome present in this company?" (Review list)

2.2 "What about the opposite - enablers or supports to the system?" (Review list of enablers)

Part Three

Effectiveness of system(s)

3.1 "Are there reviews conducted on the system?" "Why is that"?

Establish interviewee's view on the success of the current innovation process

3.2 "What is your views on the system currently used by the company?"

3.3 "How successful is the system in realising innovation value?" 
Thank the interviewee and check if any questions or concerns. Invite them to contact the interviewer if anything further occurs to them. 


\section{APPENDIX D}

\section{List of Barriers and Enablers to Innovation}

provided to Interviewees 


\section{Barriers to the internal innovation-to-outcome journey}

1. Competing management priorities due to finite resources/resource constraints.

2. Mis-allocation of (limited) investment finances.

3. Rewards and incentives unsuitable or limited in recognising innovation.

4. Encouraging status quo; turf protection, protecting existing practises.

5. Management inability to refuse a concept or identify best choice tying up critical resources better allocated to other innovative projects.

6. Bandwagon effect: where too many people want to be part of the innovation activity.

7. Fear that innovation will cannibalise existing products.

8. Management inability to implement innovation, middle and even senior managers who do not understand that the company wants and needs innovation.

9. Processes that do not support change, including lack of crossdivision/functionality structures (ie. inability to support the process).

10. Innovation introducing contradictions, managers needing to appease the need for hierarchy whilst encouraging creative anarchy; control versus flexibility, continuity among chaos.

11. Decision making by consensus.

12. Multiplicity of meetings to decide merit or to evaluate.

13. Preoccupation with current activities and markets.

14. Short term focus and/or an excessively internal focus. 
15. (Inappropriate) Strategic orientation.

16. Internal politics.

17. Organisational resistance to change and/or cultural inertia, satisfaction with status quo or guarding territories (hostile bureaucracy).

18. Lack of support to adopt change. 


\section{Facilitators or Enablers to the internal innovation-to- outcome journey}

1. Having sufficient resources to support innovations.

2. Access to resources to develop innovative ideas not requiring senior level approval.

3. Rewarding successful innovation (cash bonuses and other incentives).

4. Overarching or multi-disciplinary team to drive process.

5. Having a champion or ambassador in management to support and drive the process.

6. Training staff to encourage innovativeness or responsiveness to innovation.

7. Drawing on customers' experience to generate new ideas.

8. Interrelationship with the customer by backroom personnel.

9. Free flow of information, resources crossing departmental boundaries and hierarchy.

10. Protecting innovation from bureaucratic limitations.

11. Setting goals for innovative achievement (eg. linking results with sales strategy).

12. Encouraging a long-term perspective, linking with clarity of innovation strategy (strategic orientation).

13. Entrepreneurial and management culture supportive of innovation vision, openness to new ideas, open communication. Innovation framed as positive. 
14. Cultural pride in company's innovative achievements, cohesion.

15. Accepting failure (overcoming the fear of mistakes in being creative, being comfortable with change and conflict).

16. Organisational structure and cross functionality of divisions.

17. Relevance or advantage of innovation to individual perspective.

18. Innovation introduced as complementary to previous, successful innovations (addressing individual's reluctance to change).

19. Participative or team style of management supporting subordinates. 


\section{APPENDIX E}

Compilation of Case Study Companies'

Basic Systems 

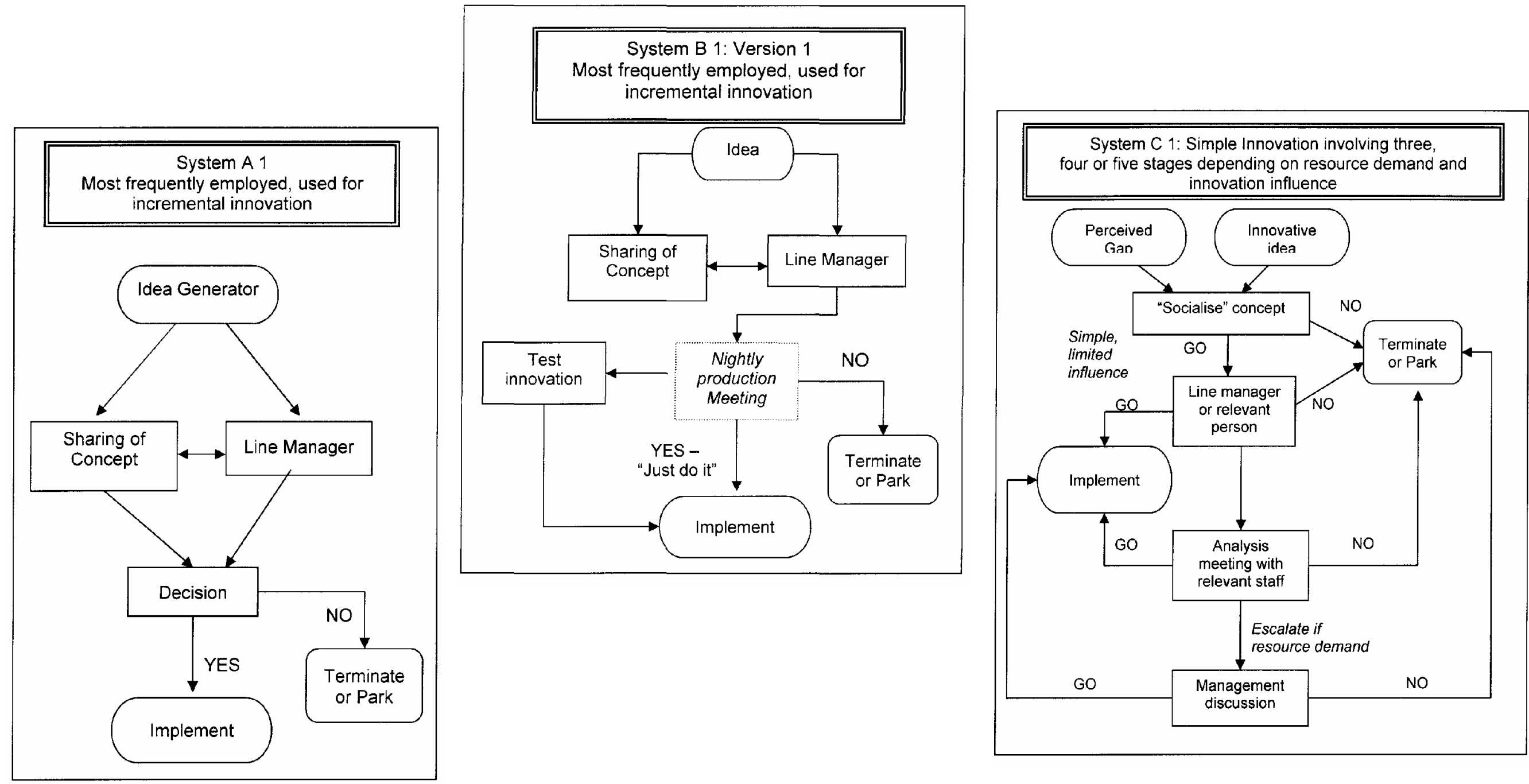


\section{APPENDIX F}

Compilation of Case Study Companies'

Radical Systems 

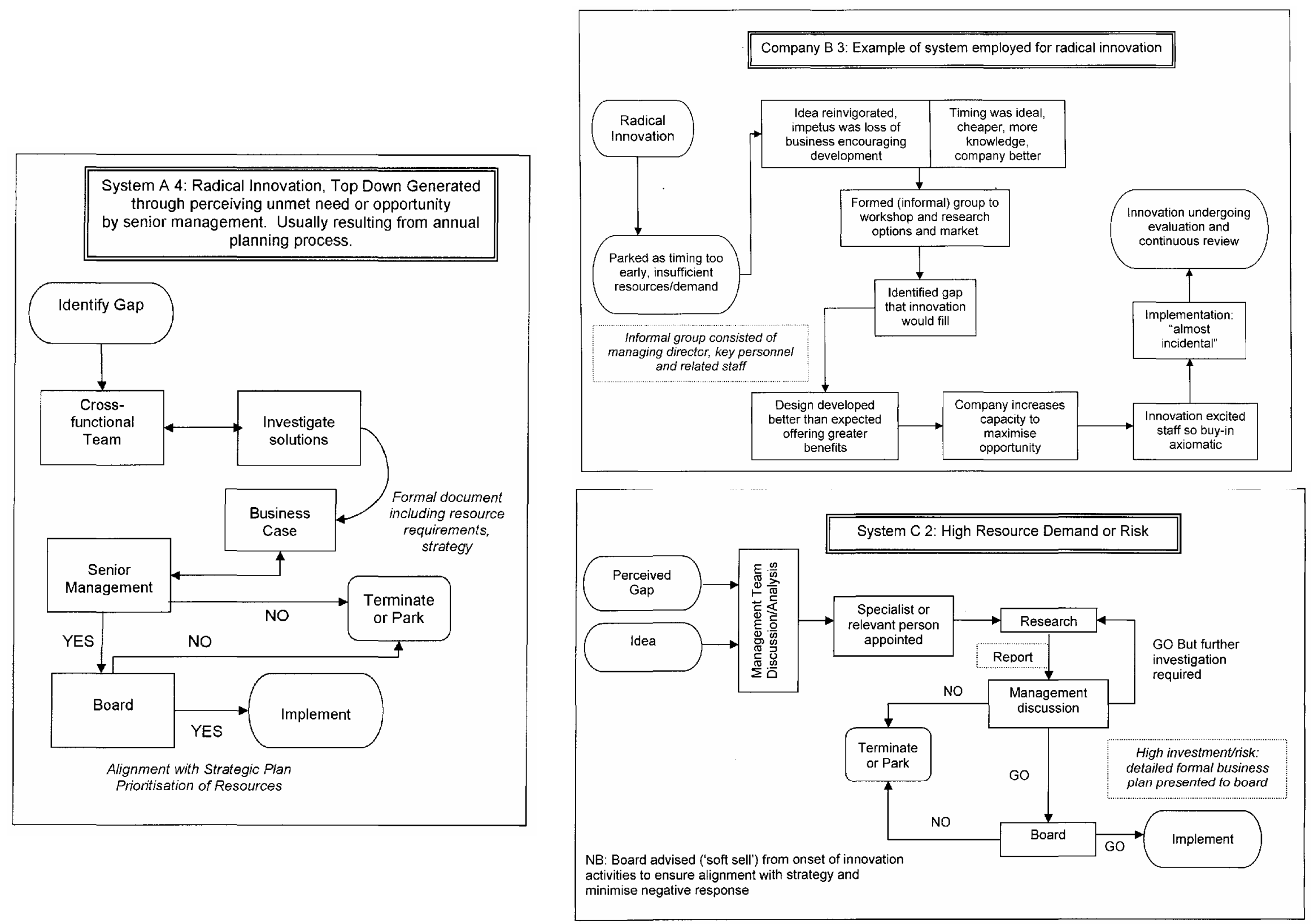